\title{
Control of Emissive Excited States of Silver(I) Halogenido Coordination Polymers by a Solid Solution Approach
}

\author{
Masaaki Dosen, Yoshitaka Kawada, Seiko Shibata, Kiyoshi Tsuge, Yoichi Sasaki, \\ Atsushi Kobayashi, Masako Kato, Shoji Ishizaka, and Noboru Kitamura
}

\section{Supporting Information}

Table S1. Reaction conditions and elemental analysis results of mixed-halogenido compounds $\left[\left\{\mathrm{Ag}_{2}\left(\mathrm{I}_{(1-x)} \mathrm{Br}_{x}\right)_{2}\left(\mathrm{PPh}_{3}\right)_{2}\right\}(\mathrm{bpy})\right]_{n}$.

Table S2. Reaction conditions and elemental analysis results of mixed-halogenido compounds $\left[\left\{\mathrm{Ag}_{2}\left(\mathrm{Br}_{(1-x)} \mathrm{Cl}_{x}\right)_{2}\left(\mathrm{PPh}_{3}\right)_{2}\right\} \text { (bpy) }\right]_{n}$.

Table S3. Reaction conditions and elemental analysis results of mixed-halogenido compounds $\left[\left\{\mathrm{Ag}_{2}\left(\mathrm{I}_{(1-x)} \mathrm{Cl}_{x}\right)_{2}\left(\mathrm{PPh}_{3}\right)_{2}\right\}(\text { bpy })\right]_{n}$.

Table S4. Crystallographic data of homo- and mixed-halogeno compounds $\left[\left\{\operatorname{Ag}_{2}\left(\mathrm{I}_{(1-}\right.\right.\right.$ $\left.\left.\left.{ }_{x)} \mathrm{Br}_{x}\right)_{2}\left(\mathrm{PPh}_{3}\right)_{2}\right\}(\mathrm{bpy})\right]_{n}$.

Table S5. Crystallographic data of homo- and mixed-halogeno compounds $\left[\left\{\mathrm{Ag}_{2}\left(\mathrm{Br}_{(1-x)} \mathrm{Cl}_{x}\right)_{2}\left(\mathrm{PPh}_{3}\right)_{2}\right\}(\text { bpy })\right]_{n}$.

Table S6. Crystallographic data of homo- and mixed-halogeno compounds $\left[\left\{\operatorname{Ag}_{2}\left(\mathrm{I}_{(1-}\right.\right.\right.$ $\left.\left.{ }_{x)} \mathrm{Cl}_{x}\right)_{2}\left(\mathrm{PPh}_{3}\right)_{2}\right\}$ (bpy) $]_{n}$.

Table S7. Energy and components of molecular orbitals around Fermi energy for the model complex $\left[\mathrm{Ag}_{2} \mathrm{I}_{2}\left(\mathrm{PPh}_{3}\right)_{2}(\mathrm{bpy})_{2}\right](\mathbf{1}-\mathrm{I})$.

Table S8. Energy and components of molecular orbitals around Fermi energy for the model complex [py- $\left\{\mathrm{Ag}_{2} \mathrm{I}_{2}\left(\mathrm{PPh}_{3}\right)_{2}\right\}$-bpy- $\left\{\mathrm{Ag}_{2} \mathrm{I}_{2}\left(\mathrm{PPh}_{3}\right)_{2}\right\}$-py] (2-I).

Table S9. Energy and components of molecular orbitals around Fermi energy for the model complex [py- $\left\{\mathrm{Ag}_{2} \mathrm{Br}_{2}\left(\mathrm{PPh}_{3}\right)_{2}\right\}$-bpy- $\left\{\mathrm{Ag}_{2} \mathrm{Br}_{2}\left(\mathrm{PPh}_{3}\right)_{2}\right\}$-py] (2-Br).

Table S10. Energy and components of molecular orbitals around Fermi energy for the model complex [py- $\left\{\mathrm{Ag}_{2} \mathrm{Cl}_{2}\left(\mathrm{PPh}_{3}\right)_{2}\right\}$-bpy- $\left\{\mathrm{Ag}_{2} \mathrm{Cl}_{2}\left(\mathrm{PPh}_{3}\right)_{2}\right\}$-py] (2-Cl).

Table S11. Energy and components of molecular orbitals around Fermi energy for the $\begin{array}{ll}\text { model complex } & {\left[\left\{\left(\mathrm{NH}_{3}\right)\left(\mathrm{PH}_{3}\right)\left(\mathrm{Ag}_{2} \mathrm{I}_{2}\right)\left(\mathrm{PPh}_{3}\right)\right\} \text {-bpy- }\right.} \\ \left.\left\{\left(\mathrm{PPh}_{3}\right)\left(\mathrm{Ag}_{2} \mathrm{I}_{2}\right)\left(\mathrm{NH}_{3}\right)\left(\mathrm{PH}_{3}\right)\right\}\right](3-\mathbf{I}) . & \end{array}$ 
Table S12. Energy and components of excited states for the model complex $\left[\left\{\left(\mathrm{NH}_{3}\right)\left(\mathrm{PH}_{3}\right)\left(\mathrm{Ag}_{2} \mathrm{I}_{2}\right)\left(\mathrm{PPh}_{3}\right)\right\}\right.$-bpy- $\left.\left\{\left(\mathrm{PPh}_{3}\right)\left(\mathrm{Ag}_{2} \mathrm{I}_{2}\right)\left(\mathrm{NH}_{3}\right)\left(\mathrm{PH}_{3}\right)\right\}\right](3-\mathrm{I})$.

Table S13. Energy and components of molecular orbitals around Fermi energy for the model complex $\quad\left[\left\{\left(\mathrm{NH}_{3}\right)\left(\mathrm{PH}_{3}\right)\left(\mathrm{Ag}_{2} \mathrm{Br}_{2}\right)\left(\mathrm{PPh}_{3}\right)\right\}\right.$-bpy$\left.\left\{\left(\mathrm{PPh}_{3}\right)\left(\mathrm{Ag}_{2} \mathrm{Br}_{2}\right)\left(\mathrm{NH}_{3}\right)\left(\mathrm{PH}_{3}\right)\right\}\right](3-\mathrm{Br})$.

Table S14. Energy and components of excited states for the model complex $\left[\left\{\left(\mathrm{NH}_{3}\right)\left(\mathrm{PH}_{3}\right)\left(\mathrm{Ag}_{2} \mathrm{Br}_{2}\right)\left(\mathrm{PPh}_{3}\right)\right\}\right.$-bpy- $\left.\left\{\left(\mathrm{PPh}_{3}\right)\left(\mathrm{Ag}_{2} \mathrm{Br}_{2}\right)\left(\mathrm{NH}_{3}\right)\left(\mathrm{PH}_{3}\right)\right\}\right](\mathbf{3}-\mathrm{Br})$.

Table S15. Energy and components of molecular orbitals around Fermi energy for the model complex $\quad\left[\left\{\left(\mathrm{NH}_{3}\right)\left(\mathrm{PH}_{3}\right)\left(\mathrm{Ag}_{2} \mathrm{Cl}_{2}\right)\left(\mathrm{PPh}_{3}\right)\right\}\right.$-bpy$\left.\left\{\left(\mathrm{PPh}_{3}\right)\left(\mathrm{Ag}_{2} \mathrm{Cl}_{2}\right)\left(\mathrm{NH}_{3}\right)\left(\mathrm{PH}_{3}\right)\right\}\right](3-\mathrm{Cl})$.

Table S16. Energy and components of excited states for the model complex $\left[\left\{\left(\mathrm{NH}_{3}\right)\left(\mathrm{PH}_{3}\right)\left(\mathrm{Ag}_{2} \mathrm{Cl}_{2}\right)\left(\mathrm{PPh}_{3}\right)\right\}\right.$-bpy- $\left.\left\{\left(\mathrm{PPh}_{3}\right)\left(\mathrm{Ag}_{2} \mathrm{Cl}_{2}\right)\left(\mathrm{NH}_{3}\right)\left(\mathrm{PH}_{3}\right)\right\}\right](\mathbf{3}-\mathbf{C l})$.

Table S17. Energy and components of molecular orbitals around Fermi energy for the model complex $\quad\left[\left\{\left(\mathrm{NH}_{3}\right)\left(\mathrm{PH}_{3}\right)\left(\mathrm{Ag}_{2} \mathrm{I}_{2}\right)\left(\mathrm{PPh}_{3}\right)\right\}\right.$-bpy$\left.\left\{\left(\mathrm{PPh}_{3}\right)\left(\mathrm{Ag}_{2} \mathrm{I}_{2}\right)\left(\mathrm{NH}_{3}\right)\left(\mathrm{PH}_{3}\right)\right\}\right](\mathbf{3}-\mathrm{I})$ in DMSO.

Table S18. Energy and components of excited states for the model complex $\left[\left\{\left(\mathrm{NH}_{3}\right)\left(\mathrm{PH}_{3}\right)\left(\mathrm{Ag}_{2} \mathrm{I}_{2}\right)\left(\mathrm{PPh}_{3}\right)\right\}\right.$-bpy- $\left.\left\{\left(\mathrm{PPh}_{3}\right)\left(\mathrm{Ag}_{2} \mathrm{I}_{2}\right)\left(\mathrm{NH}_{3}\right)\left(\mathrm{PH}_{3}\right)\right\}\right] \quad$ (3-I) in DMSO.

Table S19. Energy and components of molecular orbitals around Fermi energy for the model complex $\quad\left[\left\{\left(\mathrm{NH}_{3}\right)\left(\mathrm{PH}_{3}\right)\left(\mathrm{Ag}_{2} \mathrm{Br}_{2}\right)\left(\mathrm{PPh}_{3}\right)\right\}\right.$-bpy$\left.\left\{\left(\mathrm{PPh}_{3}\right)\left(\mathrm{Ag}_{2} \mathrm{Br}_{2}\right)\left(\mathrm{NH}_{3}\right)\left(\mathrm{PH}_{3}\right)\right\}\right](3-\mathrm{Br})$ in DMSO.

Table S20. Energy and components of excited states for the model complex $\left[\left\{\left(\mathrm{NH}_{3}\right)\left(\mathrm{PH}_{3}\right)\left(\mathrm{Ag}_{2} \mathrm{Br}_{2}\right)\left(\mathrm{PPh}_{3}\right)\right\}\right.$-bpy- $\left.\left\{\left(\mathrm{PPh}_{3}\right)\left(\mathrm{Ag}_{2} \mathrm{Br}_{2}\right)\left(\mathrm{NH}_{3}\right)\left(\mathrm{PH}_{3}\right)\right\}\right]$ (3-Br) in DMSO.

Table S21. Energy and components of molecular orbitals around Fermi energy for the model complex $\quad\left[\left\{\left(\mathrm{NH}_{3}\right)\left(\mathrm{PH}_{3}\right)\left(\mathrm{Ag}_{2} \mathrm{Cl}_{2}\right)\left(\mathrm{PPh}_{3}\right)\right\}\right.$-bpy$\left.\left\{\left(\mathrm{PPh}_{3}\right)\left(\mathrm{Ag}_{2} \mathrm{Cl}_{2}\right)\left(\mathrm{NH}_{3}\right)\left(\mathrm{PH}_{3}\right)\right\}\right](3-\mathrm{Cl})$ in DMSO.

Table S22. Energy and components of excited states for the model complex $\left[\left\{\left(\mathrm{NH}_{3}\right)\left(\mathrm{PH}_{3}\right)\left(\mathrm{Ag}_{2} \mathrm{Cl}_{2}\right)\left(\mathrm{PPh}_{3}\right)\right\}\right.$-bpy- $\left.\left\{\left(\mathrm{PPh}_{3}\right)\left(\mathrm{Ag}_{2} \mathrm{Cl}_{2}\right)\left(\mathrm{NH}_{3}\right)\left(\mathrm{PH}_{3}\right)\right\}\right] \quad(3-\mathrm{Cl})$ in DMSO.

Table S23. Relative energy to LUMO ( $\Delta)$ in complexes $\left[\left\{\left(\mathrm{NH}_{3}\right)\left(\mathrm{PH}_{3}\right)\left(\mathrm{Ag}_{2} \mathrm{X}_{2}\right)\left(\mathrm{PPh}_{3}\right)\right\}\right.$-bpy- $\left.\left\{\left(\mathrm{PPh}_{3}\right)\left(\mathrm{Ag}_{2} \mathrm{X}_{2}\right)\left(\mathrm{NH}_{3}\right)\left(\mathrm{PH}_{3}\right)\right\}\right](\mathbf{3}-\mathbf{X})$ in 
vacuum.

Table S24. Relative energy to LUMO ( $\Delta)$ in complexes $\left[\left\{\left(\mathrm{NH}_{3}\right)\left(\mathrm{PH}_{3}\right)\left(\mathrm{Ag}_{2} \mathrm{X}_{2}\right)\left(\mathrm{PPh}_{3}\right)\right\}\right.$-bpy- $\left.\left\{\left(\mathrm{PPh}_{3}\right)\left(\mathrm{Ag}_{2} \mathrm{X}_{2}\right)\left(\mathrm{NH}_{3}\right)\left(\mathrm{PH}_{3}\right)\right\}\right](\mathbf{3}-\mathbf{X})$ in DMSO.

Table S25. Energy and components of molecular orbitals around Fermi energy for the model complex $\quad\left[\left\{\left(\mathrm{NH}_{3}\right)\left(\mathrm{PH}_{3}\right)\left(\mathrm{Ag}_{2} \mathrm{Cl}_{2}\right)\left(\mathrm{PPh}_{3}\right)\right\}\right.$-bpy$\left.\left\{\left(\mathrm{PPh}_{3}\right)\left(\mathrm{Ag}_{2} \mathrm{Cl}_{2}\right)\left(\mathrm{NH}_{3}\right)\left(\mathrm{PH}_{3}\right)\right\}\right]$ with planar bpy (4-Cl) in DMSO.

Table S26. Energy and components of excited states for the model complex $\left[\left\{\left(\mathrm{NH}_{3}\right)\left(\mathrm{PH}_{3}\right)\left(\mathrm{Ag}_{2} \mathrm{Cl}_{2}\right)\left(\mathrm{PPh}_{3}\right)\right\}\right.$-bpy- $\left.\left\{\left(\mathrm{PPh}_{3}\right)\left(\mathrm{Ag}_{2} \mathrm{Cl}_{2}\right)\left(\mathrm{NH}_{3}\right)\left(\mathrm{PH}_{3}\right)\right\}\right] \quad$ with planar bpy (4-CI) in DMSO.

Figure S1. The IR absorption spectrum of $\left[\mathrm{Ag}_{2} \mathrm{I}_{2}\left(\mathrm{PPh}_{3}\right)_{2}\right.$ (bpy)] (a) together with that of bpy (b) and $\mathrm{PPh}_{3}(\mathrm{c})$.

Figure S2. Dependence of excitation (a, c, e) and emission spectra (b, d, f) of $\left[\mathrm{Ag}_{2} \mathrm{X}_{2}\left(\mathrm{PPh}_{3}\right)_{2}(\mathrm{bpy})\right]_{n}$ upon $\lambda_{\mathrm{em}}$ and $\lambda_{\text {ex. }}$ a) $\mathrm{X}=\mathrm{I}$, with $\lambda_{\mathrm{em}}$ at $420 \mathrm{~nm}$ (violet), $450 \mathrm{~nm}$ (sky blue), $500 \mathrm{~nm}$ (green); b) $\mathrm{X}=\mathrm{I}$, with $\lambda_{\text {ex }}$ at $330 \mathrm{~nm}$ (violet), $360 \mathrm{~nm}$ (sky blue), $400 \mathrm{~nm}$ (orange), $420 \mathrm{~nm}$ (red); c) $\mathrm{X}=\mathrm{Br}$, with $\lambda_{\mathrm{em}}$ at $420 \mathrm{~nm}$ (violet), $450 \mathrm{~nm}$ (sky blue), $520 \mathrm{~nm}$ (green), $530 \mathrm{~nm}$ (orange); d) $\mathrm{X}=\mathrm{Br}$, with $\lambda_{\text {ex }}$ at $310 \mathrm{~nm}$ (violet), $350 \mathrm{~nm}$ (blue), $370 \mathrm{~nm}$ (sky blue), $390 \mathrm{~nm}$ (green), $400 \mathrm{~nm}$ (orange), $410 \mathrm{~nm}$ (pink), $430 \mathrm{~nm}$ (red); e) $\mathrm{X}=\mathrm{Cl}$, with $\lambda_{\mathrm{em}}$ at $420 \mathrm{~nm}$ (violet), $450 \mathrm{~nm}$ (sky blue), $550 \mathrm{~nm}$ (orange), $600 \mathrm{~nm}$ (red); f) $\mathrm{X}=\mathrm{Cl}$ with $\lambda_{\text {ex }}$ at $300 \mathrm{~nm}$ (violet), $340 \mathrm{~nm}$ (blue), $360 \mathrm{~nm}$ (sky blue), $370 \mathrm{~nm}$ (green), $380 \mathrm{~nm}$ (orange), $400 \mathrm{~nm}$ (pink), $410 \mathrm{~nm}$ (red). (*) Artifact.

Figure S3. Emission decays of $\left[\mathrm{Ag}_{2} \mathrm{X}_{2}\left(\mathrm{PPh}_{3}\right)_{2}(\mathrm{bpy})\right]$ at temperatures of $80 \mathrm{~K}$ (blue), $170 \mathrm{~K}$ (green), $230 \mathrm{~K}$ (orange), and $290 \mathrm{~K}$ (red); a): $\mathrm{X}=\mathrm{I}, \mathrm{b}$ ): $\mathrm{X}=\mathrm{Br}, \mathrm{c}$ ): $\mathrm{X}=\mathrm{Cl}$.

Figure S4. Absorption (a) and excitation (b) spectra of $\left[\mathrm{Ag}_{2} \mathrm{X}_{2}\left(\mathrm{PPh}_{3}\right)_{2}(\mu \text {-bpy) }]_{n}\right.$ in the solid state at room temperature; X: I (red), X: Br (green), X: Cl (blue).

Figure S5. Energy of MOs of 2-X (filled circle) and 3-X (open circle). $\mathrm{X}=\mathrm{I}$ : red; $\mathrm{X}$ $=\mathrm{Br}$ : green; $\mathrm{X}=\mathrm{Cl}$ : blue.

Figure S6. Surface diagrams of MOs corresponding to $\pi$ orbitals of bpy in 3-Cl in 
DMSO with an isovalue of 0.04. a): LUMO, b): H-28, c): H-29, d): H-30.

Figure S7. Selected surface diagrams of MOs of 3-Cl (left) and 4-Cl (right) with an isovalue of 0.04 .

Scheme S1. Schematic diagram of the model complexes used in the calculations together with that of $\left[\mathrm{Ag}_{2} \mathrm{X}_{2}\left(\mathrm{PPh}_{3}\right)_{2}(\mu \text {-bpy) }]_{n}(\mathrm{a}) ;(\mathrm{b}):\left[\mathrm{Ag}_{2} \mathrm{I}_{2}\left(\mathrm{PPh}_{3}\right)_{2}(\mathrm{bpy})_{2}\right]\right.$ (1-I); (c): [py- $\left\{\mathrm{Ag}_{2} \mathrm{X}_{2}\left(\mathrm{PPh}_{3}\right)_{2}\right\}$-bpy- $\left\{\mathrm{Ag}_{2} \mathrm{X}_{2}\left(\mathrm{PPh}_{3}\right)_{2}\right\}$-py] (2-X); (d) $\left[\left\{\left(\mathrm{NH}_{3}\right)\left(\mathrm{PH}_{3}\right)\left(\mathrm{Ag}_{2} \mathrm{X}_{2}\right)\left(\mathrm{PPh}_{3}\right)\right\}-\right.$ bpy- $\left.\left\{\left(\mathrm{PPh}_{3}\right)\left(\mathrm{Ag}_{2} \mathrm{X}_{2}\right)\left(\mathrm{NH}_{3}\right)\left(\mathrm{PH}_{3}\right)\right\}\right](\mathbf{3}-\mathbf{X}, \mathbf{4 -}$ Cl). 
Table S1. Reaction conditions and elemental analysis results of mixed-halogenido compounds $\left[\left\{\operatorname{Ag}_{2}\left(\mathrm{I}_{(1-x)} \mathrm{Br}_{x}\right)_{2}\left(\mathrm{PPh}_{3}\right)_{2}\right\}(\mathrm{bpy})\right]_{n}{ }^{a}$

\begin{tabular}{|c|c|c|c|c|c|c|c|c|c|}
\hline \multicolumn{4}{|c|}{ Reaction conditions and yields } & \multicolumn{6}{|c|}{ Analysis results } \\
\hline \multirow{2}{*}{ x_reaction } & \multirow{2}{*}{$\begin{array}{c}\text { Amount of } \\
\text { AgI soln./ } \\
\text { ml } \\
(\mathrm{AgI} / \\
\mu \mathrm{mol})\end{array}$} & \multirow{2}{*}{$\begin{array}{c}\text { Amount of } \\
\text { AgBr } \\
\text { soln./ml } \\
(\mathrm{AgBr} / \\
\mu \mathrm{mol})\end{array}$} & \multirow{2}{*}{$\begin{array}{c}\text { Yield/ } \\
\text { mg } \\
(\%)\end{array}$} & \multicolumn{4}{|c|}{ Elemental analysis $(\%)$} & \multirow{2}{*}{$x \_E A$} & \multirow{2}{*}{$x \_c r y$} \\
\hline & & & & & $\mathrm{C}$ & $\mathrm{H}$ & $\mathrm{N}$ & & \\
\hline \multirow[t]{2}{*}{0.1} & 18.0 & 3.0 & 13.7 & Obsd. & 48.32 & 3.44 & 2.46 & 0.1 & 0.1 \\
\hline & $(29.8)$ & (3.3) & $(36)$ & Calcd. $(x=0.1)$ & 48.43 & 3.36 & 2.46 & & \\
\hline \multirow[t]{2}{*}{0.3} & 14.0 & 9.0 & 6.8 & Obsd. & 49.12 & 3.46 & 2.48 & 0.3 & 0.3 \\
\hline & $(23.2)$ & $(9.9)$ & (18) & Calcd. $(x=0.3)$ & 49.24 & 3.41 & 2.50 & & \\
\hline \multirow[t]{2}{*}{0.5} & 10.0 & 15.0 & 5.2 & Obsd. & 49.89 & 3.39 & 2.57 & 0.5 & 0.5 \\
\hline & $(16.6)$ & $(16.6)$ & (14) & Calcd. $(x=0.5)$ & 50.08 & 3.47 & 2.54 & & \\
\hline \multirow[t]{2}{*}{0.7} & 6.0 & 21.0 & 14.2 & Obsd. & 50.86 & 3.57 & 2.55 & 0.7 & 0.7 \\
\hline & $(9.9)$ & $(23.2)$ & $(40)$ & Calcd. $(x=0.7)$ & 50.94 & 3.53 & 2.58 & & \\
\hline \multirow[t]{2}{*}{0.9} & 2.0 & 27.0 & 14.1 & Obsd. & 51.75 & 3.60 & 2.59 & 0.9 & 0.9 \\
\hline & (3.3) & $(29.8)$ & (40) & Calcd. $(x=0.9)$ & 51.84 & 3.59 & 2.63 & & \\
\hline
\end{tabular}

${ }^{a} x_{-}$reaction: Molar fraction of $\mathrm{Br}$ in the reaction; $x \_E A$ : Molar fraction of $\mathrm{Br}$ determined by $\mathrm{EA} ; x_{-}$cry: Molar fraction of $\mathrm{Br}$ determined by X-ray analysis. 
Table S2. Reaction conditions and elemental analysis results of mixed-halogenido compounds $\left.\left[\operatorname{Ag}_{2}\left(\operatorname{Br}_{(1-x)} \mathrm{Cl}_{x}\right)_{2}\left(\mathrm{PPh}_{3}\right)_{2}\right\}(\mathrm{bpy})\right]_{n}{ }^{a}$

\begin{tabular}{|c|c|c|c|c|c|c|c|c|c|}
\hline \multicolumn{4}{|c|}{ Reaction conditions and yields } & \multicolumn{6}{|c|}{ Analysis results } \\
\hline \multirow{2}{*}{ x_reaction } & \multirow{2}{*}{$\begin{array}{c}\text { Amount of } \\
\text { AgBr } \\
\text { soln./ ml } \\
(\mathrm{AgBr} / \\
\mu \mathrm{mol})\end{array}$} & \multirow{2}{*}{$\begin{array}{c}\text { Amount of } \\
\mathrm{AgCl} \\
\text { soln./ml } \\
(\mathrm{AgCl} / \\
\mu \mathrm{mol})\end{array}$} & \multirow{2}{*}{$\begin{array}{l}\text { Yield/ } \\
\mathrm{mg} \\
(\%)\end{array}$} & \multicolumn{4}{|c|}{ Elemental analysis (\%) } & \multirow{2}{*}{$x \_E A$} & \multirow{2}{*}{$x_{-}$cry } \\
\hline & & & & & $\mathrm{C}$ & $\mathrm{H}$ & $\mathrm{N}$ & & \\
\hline 0.1 & $\begin{array}{c}14.4 \\
(41.9)\end{array}$ & $\begin{array}{c}0.6 \\
(4.7)\end{array}$ & $\begin{array}{l}9.9 \\
(20)\end{array}$ & $\begin{array}{c}\text { Obsd. } \\
\text { Calcd. }(x=0.1)\end{array}$ & $\begin{array}{l}52.59 \\
52.75\end{array}$ & $\begin{array}{l}3.63 \\
3.66\end{array}$ & $\begin{array}{l}2.68 \\
2.67\end{array}$ & 0.1 & 0.1 \\
\hline 0.3 & $\begin{array}{c}11.2 \\
(32.6)\end{array}$ & $\begin{array}{c}1.8 \\
(14.0)\end{array}$ & $\begin{array}{l}13.3 \\
(27)\end{array}$ & $\begin{array}{c}\text { Obsd. } \\
\text { Calcd. }(x=0.2)\end{array}$ & $\begin{array}{l}53.05 \\
53.20\end{array}$ & $\begin{array}{l}3.66 \\
3.69\end{array}$ & $\begin{array}{l}2.72 \\
2.70\end{array}$ & 0.2 & 0.2 \\
\hline 0.5 & $\begin{array}{c}8.0 \\
(23.3)\end{array}$ & $\begin{array}{c}3.0 \\
(23.3)\end{array}$ & $\begin{array}{l}14.4 \\
(30)\end{array}$ & $\begin{array}{c}\text { Obsd. } \\
\text { (Calcd. }(x=0.3)) \\
\text { Calcd. }(x=0.4)\end{array}$ & $\begin{array}{l}54.24 \\
53.66 \\
54.13\end{array}$ & $\begin{array}{l}3.76 \\
3.72 \\
3.75\end{array}$ & $\begin{array}{l}2.79 \\
2.72 \\
2.74\end{array}$ & 0.4 & 0.3 \\
\hline 0.7 & $\begin{array}{c}4.8 \\
(14.0)\end{array}$ & $\begin{array}{c}4.2 \\
(32.6)\end{array}$ & $\begin{array}{l}9.2 \\
(20)\end{array}$ & $\begin{array}{c}\text { Obsd. } \\
\text { Calcd. }(x=0.6) \\
\text { (Calcd. }(x=0.7))\end{array}$ & $\begin{array}{l}55.25 \\
55.09 \\
55.58\end{array}$ & $\begin{array}{l}3.82 \\
3.82 \\
3.85\end{array}$ & $\begin{array}{l}2.84 \\
2.79 \\
2.82\end{array}$ & 0.6 & 0.6 \\
\hline 0.9 & $\begin{array}{c}1.6 \\
(4.7)\end{array}$ & $\begin{array}{c}5.4 \\
(41.9)\end{array}$ & $\begin{array}{l}15.5 \\
(34)\end{array}$ & $\begin{array}{c}\text { Obsd. } \\
\text { (Calcd. }(x=0.8)) \\
\text { Calcd. }(x=0.9)\end{array}$ & $\begin{array}{l}56.55 \\
56.08 \\
56.59\end{array}$ & $\begin{array}{l}4.06 \\
3.89 \\
3.92\end{array}$ & $\begin{array}{l}2.92 \\
2.84 \\
2.87\end{array}$ & 0.9 & 0.8 \\
\hline
\end{tabular}

${ }^{a} x_{-}$reaction: Molar fraction of $\mathrm{Cl}$ in the reaction; $x \_E A$ : Molar fraction of $\mathrm{Cl}$ determined by $\mathrm{EA} ; x_{-}$cry: Molar fraction of $\mathrm{Br}$ determined by X-ray analysis. 
Table S3. Reaction conditions and elemental analysis results of mixed-halogenido compounds $\left[\left\{\operatorname{Ag}_{2}\left(\mathrm{I}_{(1-x)} \mathrm{Cl}_{x}\right)_{2}\left(\mathrm{PPh}_{3}\right)_{2}\right\}(\mathrm{bpy})\right]_{n}{ }^{a}$

\begin{tabular}{|c|c|c|c|c|c|c|c|c|c|}
\hline \multicolumn{4}{|c|}{ Reaction conditions and yields } & \multicolumn{6}{|c|}{ Analysis results } \\
\hline \multirow{2}{*}{ x_reaction } & \multirow{2}{*}{$\begin{array}{c}\text { Amount of } \\
\text { AgI soln./ } \\
\text { ml } \\
(\mathrm{AgI} / \\
\mu \mathrm{mol})\end{array}$} & \multirow{2}{*}{$\begin{array}{l}\text { Amount of } \\
\text { AgCl } \\
\text { soln./ml } \\
(\mathrm{AgCl} / \\
\mu \mathrm{mol})\end{array}$} & \multirow{2}{*}{$\begin{array}{l}\text { Yield/ } \\
\mathrm{mg} \\
(\%)\end{array}$} & \multicolumn{4}{|c|}{ Elemental analysis (\%) } & \multirow{2}{*}{$x \_E A$} & \multirow{2}{*}{$x \_c r y$} \\
\hline & & & & & $\mathrm{C}$ & $\mathrm{H}$ & $\mathrm{N}$ & & \\
\hline 0.1 & $\begin{array}{c}27.0 \\
(29.8)\end{array}$ & $\begin{array}{l}0.43 \\
(3.3)\end{array}$ & $\begin{array}{l}11.9 \\
(31)\end{array}$ & $\begin{array}{c}\text { Obsd. } \\
\text { (Calcd. }(x=0.0)) \\
\text { Calcd. }(x=0.05) \\
\text { (Calcd. }(x=0.1))\end{array}$ & $\begin{array}{l}48.31 \\
48.03 \\
48.42 \\
48.81\end{array}$ & $\begin{array}{l}3.38 \\
3.33 \\
3.36 \\
3.38\end{array}$ & $\begin{array}{l}2.38 \\
2.44 \\
2.45 \\
2.47\end{array}$ & 0.05 & 0.1 \\
\hline 0.3 & $\begin{array}{c}21.0 \\
(23.2)\end{array}$ & $\begin{array}{l}1.29 \\
(10)\end{array}$ & $\begin{array}{l}8.4 \\
(23)\end{array}$ & $\begin{array}{c}\text { Obsd. } \\
\text { Calcd. }(x=0.2)\end{array}$ & $\begin{array}{l}49.60 \\
49.61\end{array}$ & $\begin{array}{l}3.43 \\
3.44\end{array}$ & $\begin{array}{l}2.56 \\
2.52\end{array}$ & 0.2 & 0.2 \\
\hline 0.5 & $\begin{array}{c}15.0 \\
(16.6)\end{array}$ & $\begin{array}{c}2.14 \\
(16.5)\end{array}$ & $\begin{array}{c}10 \\
(28)\end{array}$ & $\begin{array}{c}\text { Obsd. } \\
\text { (Calcd. }(x=0.3)) \\
\text { Calcd. }(x=0.35) \\
\text { (Calcd. }(x=0.4))\end{array}$ & $\begin{array}{l}50.85 \\
50.44 \\
50.86 \\
51.29\end{array}$ & $\begin{array}{l}3.49 \\
3.50 \\
3.53 \\
3.56\end{array}$ & $\begin{array}{l}2.62 \\
2.56 \\
2.58 \\
2.60\end{array}$ & 0.35 & 0.3 \\
\hline 0.7 & $\begin{array}{c}9.0 \\
(9.9)\end{array}$ & $\begin{array}{c}3.00 \\
(23.2)\end{array}$ & $\begin{array}{l}8.8 \\
(26)\end{array}$ & $\begin{array}{c}\text { Obsd. } \\
\text { Calcd. }(x=0.6) \\
\text { (Calcd. }(x=0.7))\end{array}$ & $\begin{array}{l}53.34 \\
53.10 \\
54.05\end{array}$ & $\begin{array}{l}3.69 \\
3.68 \\
3.75\end{array}$ & $\begin{array}{l}2.73 \\
2.69 \\
2.74\end{array}$ & 0.6 & 0.6 \\
\hline 0.9 & $\begin{array}{c}3.0 \\
(3.3)\end{array}$ & $\begin{array}{c}3.86 \\
(29.9)\end{array}$ & $\begin{array}{l}12.6 \\
(39)\end{array}$ & $\begin{array}{c}\text { Obsd. } \\
\text { (Calcd. }(x=0.8)) \\
\text { Calcd. }(x=0.9)\end{array}$ & $\begin{array}{l}55.88 \\
55.03 \\
56.05\end{array}$ & $\begin{array}{l}3.87 \\
3.81 \\
3.89\end{array}$ & $\begin{array}{l}2.86 \\
2.79 \\
2.84\end{array}$ & 0.9 & - \\
\hline
\end{tabular}

${ }^{a}{ }_{x} \_$reaction: Molar fraction of $\mathrm{Cl}$ in the reaction; $x \_E A$ : Molar fraction of $\mathrm{Cl}$ determined by $\mathrm{EA} ; x_{-}$cry: Molar fraction of $\mathrm{Br}$ determined by X-ray analysis. 
Table S4. Crystallographic data of homo- and mixed-halogenido compounds $\left[\left\{\mathrm{Ag}_{2}\left(\mathrm{I}_{(1-x)} \mathrm{Br}_{x}\right)_{2}\left(\mathrm{PPh}_{3}\right)_{2}\right\}(\mathrm{bpy})\right]_{n .}{ }^{a}$

\begin{tabular}{|c|c|c|c|c|}
\hline molar fraction of bromine & $x=0^{b}$ & $x=0.1$ & $x=0.3$ & $x=0.5$ \\
\hline formula & $\mathrm{C}_{46} \mathrm{H}_{38} \mathrm{~N}_{2} \mathrm{P}_{2} \mathrm{Ag}_{2} \mathrm{I}_{2}$ & $\mathrm{C}_{46} \mathrm{H}_{38} \mathrm{~N}_{2} \mathrm{P}_{2} \mathrm{Ag}_{2} \mathrm{Br}_{0.2} \mathrm{I}_{1.8}$ & $\mathrm{C}_{46} \mathrm{H}_{38} \mathrm{~N}_{2} \mathrm{P}_{2} \mathrm{Ag}_{2} \mathrm{Br}_{0.6} \mathrm{I}_{1.4}$ & $\mathrm{C}_{46} \mathrm{H}_{38} \mathrm{~N}_{2} \mathrm{P}_{2} \mathrm{Ag}_{2} \mathrm{BrI}$ \\
\hline formula weight & 1150.31 & 1140.91 & 1122.11 & 1103.31 \\
\hline crystal dimensions $/ \mathrm{mm}^{3}$ & $0.09 \times 0.08 \times 0.06$ & $0.10 \times 0.08 \times 0.05$ & $0.15 \times 0.10 \times 0.05$ & $0.18 \times 0.15 \times 0.08$ \\
\hline crystal system & triclinic & triclinic & triclinic & triclinic \\
\hline space group & $P \overline{1}$ & $P \overline{1}$ & $P \overline{1}$ & $P \overline{1}$ \\
\hline$a / \AA$ & $9.2073(2)$ & $9.194(3)$ & $9.164(5)$ & $9.147(3)$ \\
\hline$b / \AA$ & $9.6991(2)$ & $9.688(4)$ & $9.680(5)$ & $9.657(3)$ \\
\hline$c / \AA$ & $13.1475(3)$ & $13.136(5)$ & $13.090(7)$ & $13.074(4)$ \\
\hline$\alpha /^{\circ}$ & 104.7441(7) & $104.616(7)$ & $104.424(7)$ & $104.168(4)$ \\
\hline$\beta 1^{\circ}$ & $109.3402(7)$ & $109.430(7)$ & $109.286(8)$ & $109.230(4)$ \\
\hline$\gamma /{ }^{\circ}$ & $92.6061(7)$ & $92.596(3)$ & $92.684(4)$ & $92.971(3)$ \\
\hline$V / \AA^{3}$ & $1060.48(4)$ & $1056.9(7)$ & $1050.8(9)$ & $1046.1(5)$ \\
\hline$Z$ & 1 & 1 & 1 & 1 \\
\hline$\rho_{\text {calcd }}\left(\mathrm{g} / \mathrm{cm}^{3}\right)$ & 1.801 & 1.792 & 1.773 & 1.751 \\
\hline $2 \theta_{\max }{ }^{\circ}$ & 136.40 & 59.15 & 59.15 & 59.15 \\
\hline radiation & $\mathrm{Cu} \mathrm{K \alpha}$ & Mo K $\alpha$ & Mo K $\alpha$ & Mo K $\alpha$ \\
\hline wavelength / $\AA$ & 1.54187 & 0.7107 & 0.7107 & 0.7107 \\
\hline temperature $/{ }^{\circ} \mathrm{C}$ & -120 & -120 & -120 & -120 \\
\hline no. of refs measured & 11606 & 6723 & 6683 & 6344 \\
\hline no. of refs independent & 3798 & 4309 & 4226 & 4259 \\
\hline no. of refs with $I>2 \sigma(I)$ & 3474 & 3473 & 3208 & 3704 \\
\hline no. of variables & 244 & 245 & 245 & 245 \\
\hline R1, wR2, GOF & $0.035,0.087,1.11$ & $0.053,0.121,1.13$ & $0.062,0.156,1.15$ & $0.041,0110,1.06$ \\
\hline residual, $e^{-} / \AA^{3}$ & 1.06 & 1.62 & 1.51 & 1.62 \\
\hline
\end{tabular}


Table S4. (Continued)

\begin{tabular}{|c|c|c|c|}
\hline molar fraction of bromine & $x=0.7$ & $x=0.9$ & $x=1$ \\
\hline formula & $\mathrm{C}_{46} \mathrm{H}_{38} \mathrm{~N}_{2} \mathrm{P}_{2} \mathrm{Ag}_{2} \mathrm{Br}_{1.4} \mathrm{I}_{0.6}$ & $\mathrm{C}_{46} \mathrm{H}_{38} \mathrm{~N}_{2} \mathrm{P}_{2} \mathrm{Ag}_{2} \mathrm{Br}_{1.8} \mathrm{I}_{0.2}$ & $\mathrm{C}_{46} \mathrm{H}_{38} \mathrm{~N}_{2} \mathrm{P}_{2} \mathrm{Ag}_{2} \mathrm{Br}_{2}$ \\
\hline formula weight & 1084.51 & 1065.71 & 1056.31 \\
\hline crystal dimensions $/ \mathrm{mm}^{3}$ & $0.18 \times 0.10 \times 0.08$ & $0.25 \times 0.18 \times 0.08$ & $0.50 \times 0.20 \times 0.10$ \\
\hline crystal system & triclinic & triclinic & triclinic \\
\hline space group & $P \overline{1}$ & $P \overline{1}$ & $P \overline{1}$ \\
\hline$a / \AA$ & $9.121(6)$ & $9.093(4)$ & $9.063(7)$ \\
\hline$b / \AA$ & $9.630(6)$ & $9.600(5)$ & $9.581(4)$ \\
\hline$c / \AA$ & $13.037(9)$ & $13.017(7)$ & $13.012(7)$ \\
\hline$\alpha /^{\circ}$ & $103.937(9)$ & $103.684(10)$ & $103.52(6)$ \\
\hline$\beta 1^{\circ}$ & $109.099(10)$ & $108.830(5)$ & $108.49(5)$ \\
\hline$\gamma /{ }^{\circ}$ & $93.036(4)$ & $93.321(5)$ & $93.54(7)$ \\
\hline$V / \AA^{3}$ & $1039.4(11)$ & $1033.7(8)$ & $1030.5(12)$ \\
\hline$Z$ & 1 & 1 & 1 \\
\hline$\rho_{\text {calcd }}\left(\mathrm{g} / \mathrm{cm}^{3}\right)$ & 1.732 & 1.712 & 1.702 \\
\hline $2 \theta_{\max } /{ }^{\circ}$ & 59.15 & 59.15 & 59.15 \\
\hline radiation & Mo K $\alpha$ & Mo K $\alpha$ & Mo K $\alpha$ \\
\hline wavelength / $\AA$ & 0.7107 & 0.7107 & 0.7107 \\
\hline temperature $/{ }^{\circ} \mathrm{C}$ & -120 & -120 & -120 \\
\hline no. of refs measured & 6568 & 6478 & 6361 \\
\hline no. of refs independent & 4377 & 4186 & 4240 \\
\hline no. of refs with $I>2 \sigma(I)$ & 3014 & 3337 & 3622 \\
\hline no. of variables & 245 & 245 & 244 \\
\hline R1, wR2, GOF & $0.069,0.213,1.14$ & $0.061,0.155,1.10$ & $0.049,0.125,1.00$ \\
\hline residual, $e^{-} / \AA^{3}$ & 1.91 & 1.61 & 1.02 \\
\hline
\end{tabular}


${ }^{a} \mathrm{R} 1=\Sigma|| F_{o}|-| F_{c} \| / \Sigma\left|F_{o}\right|$ for reflections with $I>2 \sigma(I), \mathrm{wR} 2=\left[\Sigma\left(w\left(F_{o}^{2}-F_{c}^{2}\right)^{2} / \Sigma w\left(F_{o}^{2}\right)^{2}\right)\right]^{1 / 2}$ for all reflections, GOF $=\left[\Sigma w\left(F_{o}^{2}-F_{c}{ }^{2}\right)^{2} /\left(N_{o^{-}}\right.\right.$ $\left.\left.N_{v}\right)\right]^{1 / 2}$ for all reflections where $N_{o}$ and $N_{v}$ are number of independent reflections and variables.

${ }^{b}$ The values having reported in ref. $\mathrm{S} 1$ are listed for comparison. 
Table S5. Crystallographic data of homo- and mixed-halogenido compounds $\left[\left\{\operatorname{Ag}_{2}\left(\mathrm{Br}_{(1-x)} \mathrm{Cl}_{x}\right)_{2}\left(\mathrm{PPh}_{3}\right)_{2}\right\}(\mathrm{bpy})\right]_{n .}{ }^{a}$

\begin{tabular}{|c|c|c|c|c|}
\hline molar fraction of chlorine & $x=0$ & $x=0.1$ & $x=0.2$ & $x=0.3$ \\
\hline formula & $\mathrm{C}_{46} \mathrm{H}_{38} \mathrm{~N}_{2} \mathrm{P}_{2} \mathrm{Ag}_{2} \mathrm{Br}_{2}$ & $\mathrm{C}_{46} \mathrm{H}_{38} \mathrm{~N}_{2} \mathrm{P}_{2} \mathrm{Ag}_{2} \mathrm{Br}_{1.8} \mathrm{Cl}_{0.2}$ & $\mathrm{C}_{46} \mathrm{H}_{38} \mathrm{~N}_{2} \mathrm{P}_{2} \mathrm{Ag}_{2} \mathrm{Br}_{1.6} \mathrm{Cl}_{0.4}$ & $\mathrm{C}_{46} \mathrm{H}_{38} \mathrm{~N}_{2} \mathrm{P}_{2} \mathrm{Ag}_{2} \mathrm{Br}_{1.4} \mathrm{Cl}_{0.6}$ \\
\hline formula weight & 1056.31 & 1047.42 & 1122.11 & 1103.31 \\
\hline crystal dimensions $/ \mathrm{mm}^{3}$ & $0.50 \times 0.20 \times 0.10$ & $0.25 \times 0.13 \times 0.08$ & $0.30 \times 0.20 \times 0.05$ & $0.25 \times 0.15 \times 0.05$ \\
\hline crystal system & triclinic & triclinic & triclinic & triclinic \\
\hline space group & $P \overline{1}$ & $P \overline{1}$ & $P \overline{1}$ & $P \overline{1}$ \\
\hline$a / \AA$ & $9.063(7)$ & $9.068(3)$ & $9.065(3)$ & $9.051(3)$ \\
\hline$b / \AA$ & $9.581(4)$ & $9.588(3)$ & $9.576(4)$ & $9.562(3)$ \\
\hline$c / \AA$ & $13.012(7)$ & $13.017(4)$ & $13.003(5)$ & $13.001(5)$ \\
\hline$\alpha /^{\circ}$ & $103.52(6)$ & $103.477(4)$ & $103.358(5)$ & $103.325(7)$ \\
\hline$\beta /^{\circ}$ & $108.49(5)$ & $108.582(4)$ & $108.507(4)$ & $108.402(6)$ \\
\hline$\gamma /{ }^{\circ}$ & $93.54(7)$ & $93.493(4)$ & $93.603(5)$ & $93.732(3)$ \\
\hline$V / \AA^{3}$ & $1030.5(12)$ & $1031.9(6)$ & $1030.0(6)$ & $1027.2(6)$ \\
\hline$Z$ & 1 & 1 & 1 & 1 \\
\hline$\rho_{\text {calcd }}\left(\mathrm{g} / \mathrm{cm}^{3}\right)$ & 1.702 & 1.685 & 1.674 & 1.664 \\
\hline $2 \theta_{\max } / 0$ & 59.15 & 59.15 & 59.15 & 59.15 \\
\hline radiation & Mo K $\alpha$ & Mo K $\alpha$ & Mo K $\alpha$ & Mo K $\alpha$ \\
\hline wavelength / $\AA$ & 0.7107 & 0.7107 & 0.7107 & 0.7107 \\
\hline temperature $/{ }^{\circ} \mathrm{C}$ & -120 & -120 & -120 & -120 \\
\hline no. of refs measured & 6361 & 6477 & 6483 & 6516 \\
\hline no. of refs independent & 4240 & 4304 & 4308 & 4215 \\
\hline no. of refs with $I>2 \sigma(I)$ & 3622 & 3309 & 3817 & 3507 \\
\hline no. of variables & 244 & 245 & 245 & 245 \\
\hline R1, wR2, GOF & $0.049,0.125,1.00$ & $0.052,0.134,0.98$ & $0.044,0.121,1.12$ & $0.048,0.117,1.10$ \\
\hline residual, $e^{-} / \AA^{3}$ & 1.02 & 1.51 & 0.84 & 1.04 \\
\hline
\end{tabular}


Table S5. (Continued)

\begin{tabular}{|c|c|c|c|}
\hline molar fraction of chlorine & $x=0.6$ & $x=0.8$ & $x=1$ \\
\hline formula & $\mathrm{C}_{46} \mathrm{H}_{38} \mathrm{~N}_{2} \mathrm{P}_{2} \mathrm{Ag}_{2} \mathrm{Br}_{0.8} \mathrm{Cl}_{1.2}$ & $\mathrm{C}_{46} \mathrm{H}_{38} \mathrm{~N}_{2} \mathrm{P}_{2} \mathrm{Ag}_{2} \mathrm{Br}_{0.4} \mathrm{Cl}_{1.6}$ & $\mathrm{C}_{46} \mathrm{H}_{38} \mathrm{~N}_{2} \mathrm{P}_{2} \mathrm{Ag}_{2} \mathrm{Cl}_{2}$ \\
\hline formula weight & 1002.97 & 985.19 & 967.41 \\
\hline crystal dimensions $/ \mathrm{mm}^{3}$ & $0.38 \times 0.25 \times 0.05$ & $0.38 \times 0.38 \times 0.13$ & $0.50 \times 0.43 \times 0.38$ \\
\hline crystal system & triclinic & triclinic & triclinic \\
\hline space group & $P \overline{1}$ & $P \overline{1}$ & $P \overline{1}$ \\
\hline$a / \AA$ & $9.1080(15)$ & $9.0670(16)$ & $9.048(4)$ \\
\hline$b / \AA$ & $13.730(2)$ & $13.733(3)$ & $13.756(3)$ \\
\hline$c / \AA$ & $17.477(4)$ & $17.452(4)$ & $17.423(12)$ \\
\hline$\alpha /^{\circ}$ & $71.805(10)$ & $71.657(9)$ & $71.56(6)$ \\
\hline$\beta /^{\circ}$ & $81.021(12)$ & $80.978(12)$ & $80.96(6)$ \\
\hline$\gamma /{ }^{\circ}$ & $75.904(11)$ & $75.861(12)$ & $75.85(4)$ \\
\hline$V / \AA^{3}$ & $2006.2(6)$ & $1992.5(7)$ & $1987.1(18)$ \\
\hline$Z$ & 2 & 2 & 2 \\
\hline$\rho_{\text {calcd }}\left(\mathrm{g} / \mathrm{cm}^{3}\right)$ & 1.660 & 1.642 & 1.617 \\
\hline $2 \theta_{\max } /{ }^{\circ}$ & 59.15 & 59.15 & 59.15 \\
\hline radiation & Mo K $\alpha$ & Mo K $\alpha$ & Mo K $\alpha$ \\
\hline wavelength / $\AA$ & 0.7107 & 0.7107 & 0.7107 \\
\hline temperature $/{ }^{\circ} \mathrm{C}$ & -120 & -120 & -120 \\
\hline no. of refs measured & 12611 & 12447 & 12239 \\
\hline no. of refs independent & 8217 & 8123 & 7980 \\
\hline no. of refs with $I>2 \sigma(I)$ & 6480 & 6705 & 6702 \\
\hline no. of variables & 489 & 489 & 487 \\
\hline R1, wR2, GOF & $0.053,0.138,1.14$ & $0.040,0.095,1.09$ & $0.031,0.093,1.07$ \\
\hline residual, $e^{-} / \AA^{3}$ & 1.23 & 0.71 & 0.65 \\
\hline
\end{tabular}

SI-12 
${ }^{a} \mathrm{R} 1=\Sigma|| F_{o}|-| F_{c} \| / \Sigma\left|F_{o}\right|$ for reflections with $I>2 \sigma(I), \mathrm{wR} 2=\left[\Sigma\left(w\left(F_{o}^{2}-F_{c}^{2}\right)^{2} / \Sigma w\left(F_{o}^{2}\right)^{2}\right)\right]^{1 / 2}$ for all reflections, GOF $=\left[\Sigma w\left(F_{o}^{2}-F_{c}^{2}\right)^{2} /\left(N_{o^{-}}\right.\right.$ $\left.\left.N_{v}\right)\right]^{1 / 2}$ for all reflections where $N_{o}$ and $N_{v}$ are number of independent reflections and variables. 
Table S6. Crystallographic data of homo- and mixed-halogenido compounds $\left[\left\{\mathrm{Ag}_{2}\left(\mathrm{I}_{(1-x)} \mathrm{Cl}_{x}\right)_{2}\left(\mathrm{PPh}_{3}\right)_{2}\right\}(\mathrm{bpy})\right]_{n}{ }^{a}$

\begin{tabular}{|c|c|c|c|c|}
\hline molar fraction of chlorine & $x=0^{b}$ & $x=0.1$ & $x=0.2$ & $x=0.3$ \\
\hline formula & $\mathrm{C}_{46} \mathrm{H}_{38} \mathrm{~N}_{2} \mathrm{P}_{2} \mathrm{Ag}_{2} \mathrm{I}_{2}$ & $\mathrm{C}_{46} \mathrm{H}_{38} \mathrm{~N}_{2} \mathrm{P}_{2} \mathrm{Ag}_{2} \mathrm{Cl}_{0.2} \mathrm{I}_{1.8}$ & $\mathrm{C}_{46} \mathrm{H}_{38} \mathrm{~N}_{2} \mathrm{P}_{2} \mathrm{Ag}_{2} \mathrm{Cl}_{0.4} \mathrm{I}_{1.6}$ & $\mathrm{C}_{46} \mathrm{H}_{38} \mathrm{~N}_{2} \mathrm{P}_{2} \mathrm{Ag}_{2} \mathrm{Cl}_{0.6} \mathrm{I}_{1.4}$ \\
\hline formula weight & 1150.31 & 1132.02 & 1113.73 & 1095.44 \\
\hline crystal dimensions $/ \mathrm{mm}^{3}$ & $0.09 \times 0.08 \times 0.06$ & $0.20 \times 0.08 \times 0.08$ & $0.25 \times 0.20 \times 0.08$ & $0.30 \times 0.13 \times 0.05$ \\
\hline crystal system & triclinic & triclinic & triclinic & triclinic \\
\hline space group & $P \overline{1}$ & $P \overline{1}$ & $P \overline{1}$ & $P \overline{1}$ \\
\hline$a / \AA ̊$ & $9.2073(2)$ & $9.184(3)$ & $9.176(6)$ & $9.145(3)$ \\
\hline$b / \AA$ & $9.6991(2)$ & $9.687(3)$ & $9.671(6)$ & $9.671(3)$ \\
\hline$c / \AA$ & $13.1475(3)$ & $13.131(5)$ & $13.098(8)$ & $13.101(5)$ \\
\hline$\alpha /^{\circ}$ & $104.7441(7)$ & $104.701(4)$ & $104.531(10)$ & $104.267(4)$ \\
\hline$\beta /^{\circ}$ & $109.3402(7)$ & $109.327(6)$ & $109.254(7)$ & $109.406(5)$ \\
\hline$\gamma /{ }^{\circ}$ & $92.6061(7)$ & $92.606(3)$ & $92.711(6)$ & $92.727(4)$ \\
\hline$V / \AA^{3}$ & $1060.48(4)$ & $1055.6(6)$ & $1051.5(11)$ & $1048.5(6)$ \\
\hline$Z$ & 1 & 1 & 1 & 1 \\
\hline$\rho_{\text {calcd }}\left(\mathrm{g} / \mathrm{cm}^{3}\right)$ & 1.801 & 1.781 & 1.759 & 1.735 \\
\hline $2 \theta_{\max } /{ }^{\circ}$ & 136.40 & 59.15 & 59.15 & 59.15 \\
\hline radiation & $\mathrm{Cu} \mathrm{K} \alpha$ & Mo K $\alpha$ & Mo K $\alpha$ & Mo K $\alpha$ \\
\hline wavelength / $\AA$ & 1.54187 & 0.7107 & 0.7107 & 0.7107 \\
\hline temperature $/{ }^{\circ} \mathrm{C}$ & -120 & -120 & -120 & -120 \\
\hline no. of refs measured & 11606 & 6682 & 6489 & 6440 \\
\hline no. of refs independent & 3798 & 4316 & 4386 & 4341 \\
\hline no. of refs with $I>2 \sigma(I)$ & 3474 & 3582 & 4059 & 3571 \\
\hline no. of variables & 244 & 245 & 245 & 245 \\
\hline R1, wR2, GOF & $0.035,0.087,1.11$ & $0.044,0.101,1.15$ & $0.042,0.119,1.17$ & $0.050,0.142,1.07$ \\
\hline residual, $e^{-} / \AA^{3}$ & 1.06 & 1.54 & 1.46 & 1.55 \\
\hline
\end{tabular}


Table S6. (Continued)

\begin{tabular}{|c|c|c|}
\hline molar fraction of chlorine & $x=0.6$ & $x=1$ \\
\hline formula & $\mathrm{C}_{46} \mathrm{H}_{38} \mathrm{~N}_{2} \mathrm{P}_{2} \mathrm{Ag}_{2} \mathrm{Cl}_{1.2} \mathrm{I}_{0.8}$ & $\mathrm{C}_{46} \mathrm{H}_{38} \mathrm{~N}_{2} \mathrm{P}_{2} \mathrm{Ag}_{2} \mathrm{Cl}_{2}$ \\
\hline formula weight & 1040.57 & 967.41 \\
\hline crystal dimensions $/ \mathrm{mm}^{3}$ & $0.25 \times 0.13 \times 0.08$ & $0.50 \times 0.43 \times 0.38$ \\
\hline crystal system & triclinic & triclinic \\
\hline space group & $P \overline{1}$ & $P \overline{1}$ \\
\hline$a / \AA$ & $9.080(2)$ & $9.048(4)$ \\
\hline$b / \AA$ & $9.633(3)$ & $13.756(3)$ \\
\hline$c / \AA$ & $13.047(3)$ & $17.423(12)$ \\
\hline$\alpha /^{\circ}$ & $103.685(10)$ & $71.56(6)$ \\
\hline$\beta 1^{\circ}$ & $109.202(9)$ & $80.96(6)$ \\
\hline$\gamma /{ }^{\circ}$ & $93.293(12)$ & $75.85(4)$ \\
\hline$V / \AA^{3}$ & $1035.8(4)$ & 1987.1(18) \\
\hline$Z$ & 1 & 2 \\
\hline$\rho_{\text {calcd }}\left(\mathrm{g} / \mathrm{cm}^{3}\right)$ & 1.668 & 1.617 \\
\hline $2 \theta_{\max } / 0$ & 59.15 & 59.15 \\
\hline radiation & Mo K $\alpha$ & Mo K $\alpha$ \\
\hline wavelength / $\AA$ & 0.7107 & 0.7107 \\
\hline temperature $/{ }^{\circ} \mathrm{C}$ & -120 & -120 \\
\hline no. of refs measured & 6438 & 12239 \\
\hline no. of refs independent & 4299 & 7980 \\
\hline no. of refs with $I>2 \sigma(I)$ & 3848 & 6703 \\
\hline no. of variables & 245 & 487 \\
\hline $\mathrm{R} 1, \mathrm{wR} 2, \mathrm{GOF}$ & $0.050,0.131,1.15$ & $0.031,0.093,1.07$ \\
\hline residual, $e^{-} / \AA^{3}$ & 1.30 & 0.65 \\
\hline
\end{tabular}


${ }^{a} \mathrm{R} 1=\Sigma|| F_{o}|-| F_{c} \| / \Sigma\left|F_{o}\right|$ for reflections with $I>2 \sigma(I), \mathrm{wR} 2=\left[\Sigma\left(w\left(F_{o}^{2}-F_{c}^{2}\right)^{2} / \Sigma w\left(F_{o}^{2}\right)^{2}\right)\right]^{1 / 2}$ for all reflections, GOF $=\left[\Sigma w\left(F_{o}^{2}-F_{c}{ }^{2}\right)^{2} /\left(N_{o^{-}}\right.\right.$ $\left.\left.N_{v}\right)\right]^{1 / 2}$ for all reflections where $N_{o}$ and $N_{v}$ are number of independent reflections and variables.

${ }^{b}$ The values having reported in ref. $\mathrm{S} 1$ are listed for comparison. 
Table S7. Energy and components of molecular orbitals around Fermi energy for the model complex $\left[\mathrm{Ag}_{2} \mathrm{I}_{2}\left(\mathrm{PPh}_{3}\right)_{2}(\mathrm{bpy})_{2}\right]$ (1-I).

\begin{tabular}{|c|c|c|c|c|c|c|}
\hline & $\begin{array}{c}\text { Energy } \\
(\mathrm{eV})\end{array}$ & I & $\mathrm{Ag}$ & bpy & $\mathrm{P}$ & $\mathrm{Ph}$ \\
\hline $\mathrm{L}+10$ & -0.41 & 0.00 & 0.01 & 0.00 & 0.03 & 0.95 \\
\hline L+9 & -0.46 & 0.00 & 0.00 & 0.00 & 0.04 & 0.95 \\
\hline $\mathrm{L}+8$ & -0.47 & 0.00 & 0.00 & 0.00 & 0.05 & 0.94 \\
\hline $\mathrm{L}+7$ & -0.54 & 0.00 & 0.00 & 0.89 & 0.00 & 0.09 \\
\hline $\mathrm{L}+6$ & -0.55 & 0.00 & 0.00 & 0.95 & 0.00 & 0.04 \\
\hline $\mathrm{L}+5$ & -0.66 & 0.00 & 0.01 & 0.09 & 0.05 & 0.84 \\
\hline $\mathrm{L}+4$ & -0.67 & 0.00 & 0.01 & 0.04 & 0.06 & 0.88 \\
\hline $\mathrm{L}+3$ & -1.16 & 0.00 & 0.00 & 0.99 & 0.00 & 0.00 \\
\hline $\mathrm{L}+2$ & -1.16 & 0.00 & 0.00 & 0.99 & 0.00 & 0.00 \\
\hline $\mathrm{L}+1$ & -2.15 & 0.00 & 0.00 & 0.99 & 0.00 & 0.00 \\
\hline LUMO & -2.15 & 0.00 & 0.00 & 0.99 & 0.00 & 0.00 \\
\hline HOMO & -4.86 & 0.59 & 0.19 & 0.00 & 0.13 & 0.08 \\
\hline $\mathrm{H}-1$ & -5.24 & 0.74 & 0.25 & 0.00 & 0.00 & 0.00 \\
\hline $\mathrm{H}-2$ & -5.40 & 0.87 & 0.08 & 0.00 & 0.02 & 0.03 \\
\hline $\mathrm{H}-3$ & -5.44 & 0.79 & 0.13 & 0.06 & 0.00 & 0.01 \\
\hline H-4 & -5.54 & 0.28 & 0.26 & 0.02 & 0.22 & 0.23 \\
\hline H-5 & -5.83 & 0.81 & 0.17 & 0.00 & 0.00 & 0.02 \\
\hline H-6 & -6.15 & 0.22 & 0.38 & 0.24 & 0.05 & 0.11 \\
\hline H-7 & -6.37 & 0.21 & 0.15 & 0.05 & 0.11 & 0.47 \\
\hline H-8 & -6.86 & 0.00 & 0.00 & 0.00 & 0.00 & 1.00 \\
\hline H-9 & -6.86 & 0.00 & 0.00 & 0.00 & 0.00 & 1.00 \\
\hline H-10 & -6.92 & 0.00 & 0.00 & 0.00 & 0.00 & 0.98 \\
\hline H-11 & -6.92 & 0.00 & 0.00 & 0.00 & 0.00 & 0.99 \\
\hline H-12 & -7.00 & 0.01 & 0.08 & 0.05 & 0.00 & 0.85 \\
\hline H-13 & -7.05 & 0.01 & 0.00 & 0.00 & 0.00 & 0.97 \\
\hline H-14 & -7.08 & 0.00 & 0.00 & 0.99 & 0.00 & 0.00 \\
\hline H-15 & -7.08 & 0.00 & 0.00 & 0.99 & 0.00 & 0.00 \\
\hline H-16 & -7.12 & 0.02 & 0.13 & 0.05 & 0.01 & 0.78 \\
\hline H-17 & -7.16 & 0.02 & 0.16 & 0.12 & 0.01 & 0.69 \\
\hline H-18 & -7.19 & 0.00 & 0.01 & 0.00 & 0.00 & 0.98 \\
\hline H-19 & -7.20 & 0.01 & 0.08 & 0.06 & 0.01 & 0.83 \\
\hline $\mathrm{H}-20$ & -7.23 & 0.01 & 0.13 & 0.07 & 0.00 & 0.78 \\
\hline
\end{tabular}


(Continued)

\begin{tabular}{lllllll} 
H-21 & -7.28 & 0.02 & 0.07 & 0.05 & 0.00 & 0.85 \\
H-22 & -7.48 & 0.00 & 0.22 & 0.59 & 0.02 & 0.17 \\
H-23 & -7.63 & 0.01 & 0.02 & 0.93 & 0.00 & 0.03 \\
H-24 & -7.77 & 0.00 & 0.00 & 0.99 & 0.00 & 0.00 \\
H-25 & -7.77 & 0.00 & 0.00 & 0.99 & 0.00 & 0.00 \\
H-26 & -7.82 & 0.03 & 0.30 & 0.42 & 0.07 & 0.19 \\
H-27 & -7.89 & 0.21 & 0.50 & 0.20 & 0.03 & 0.06 \\
H-28 & -7.92 & 0.00 & 0.00 & 0.99 & 0.00 & 0.00 \\
H-29 & -7.93 & 0.03 & 0.07 & 0.88 & 0.00 & 0.01 \\
H-30 & -8.09 & 0.00 & 0.94 & 0.02 & 0.00 & 0.03 \\
\hline
\end{tabular}


Table S8. Energy and components of molecular orbitals around Fermi energy for the model complex [py- $\left\{\mathrm{Ag}_{2} \mathrm{I}_{2}\left(\mathrm{PPh}_{3}\right)_{2}\right\}$-bpy- $\left\{\mathrm{Ag}_{2} \mathrm{I}_{2}\left(\mathrm{PPh}_{3}\right)_{2}\right\}$-py] (2-I).

\begin{tabular}{|c|c|c|c|c|c|c|c|}
\hline & $\begin{array}{c}\text { Energy } \\
(\mathrm{eV})\end{array}$ & I & $\mathrm{Ag}$ & bpy & $\mathrm{P}$ & $\mathrm{Ph}$ & py \\
\hline $\mathrm{L}+10$ & -0.51 & 0.00 & 0.00 & 0.75 & 0.02 & 0.00 & 0.22 \\
\hline $\mathrm{L}+9$ & -0.56 & 0.00 & 0.00 & 0.00 & 0.05 & 0.94 & 0.00 \\
\hline $\mathrm{L}+8$ & -0.57 & 0.00 & 0.00 & 0.06 & 0.05 & 0.88 & 0.00 \\
\hline $\mathrm{L}+7$ & -0.61 & 0.00 & 0.01 & 0.00 & 0.06 & 0.92 & 0.00 \\
\hline $\mathrm{L}+6$ & -0.62 & 0.00 & 0.01 & 0.16 & 0.05 & 0.76 & 0.01 \\
\hline $\mathrm{L}+5$ & -0.66 & 0.00 & 0.00 & 0.00 & 0.01 & 0.00 & 0.98 \\
\hline $\mathrm{L}+4$ & -0.66 & 0.00 & 0.00 & 0.00 & 0.01 & 0.00 & 0.98 \\
\hline $\mathrm{L}+3$ & -1.14 & 0.00 & 0.00 & 0.98 & 0.00 & 0.00 & 0.01 \\
\hline $\mathrm{L}+2$ & -1.15 & 0.00 & 0.00 & 0.00 & 0.00 & 0.00 & 0.98 \\
\hline $\mathrm{L}+1$ & -1.15 & 0.00 & 0.00 & 0.00 & 0.00 & 0.00 & 0.98 \\
\hline LUMO & -2.20 & 0.00 & 0.00 & 0.98 & 0.00 & 0.00 & 0.00 \\
\hline HOMO & -4.78 & 0.59 & 0.19 & 0.00 & 0.13 & 0.08 & 0.00 \\
\hline $\mathrm{H}-1$ & -4.78 & 0.60 & 0.19 & 0.00 & 0.13 & 0.08 & 0.00 \\
\hline $\mathrm{H}-2$ & -5.16 & 0.74 & 0.25 & 0.00 & 0.00 & 0.00 & 0.00 \\
\hline $\mathrm{H}-3$ & -5.16 & 0.74 & 0.25 & 0.00 & 0.00 & 0.00 & 0.00 \\
\hline $\mathrm{H}-4$ & -5.31 & 0.87 & 0.08 & 0.00 & 0.02 & 0.02 & 0.00 \\
\hline H-5 & -5.31 & 0.88 & 0.08 & 0.00 & 0.02 & 0.02 & 0.00 \\
\hline H-6 & -5.35 & 0.79 & 0.13 & 0.03 & 0.00 & 0.01 & 0.03 \\
\hline H-7 & -5.36 & 0.79 & 0.13 & 0.03 & 0.00 & 0.01 & 0.03 \\
\hline H-8 & -5.46 & 0.28 & 0.27 & 0.00 & 0.22 & 0.22 & 0.00 \\
\hline H-9 & -5.47 & 0.27 & 0.27 & 0.00 & 0.22 & 0.23 & 0.00 \\
\hline H-10 & -5.75 & 0.81 & 0.17 & 0.00 & 0.00 & 0.02 & 0.00 \\
\hline H-11 & -5.75 & 0.81 & 0.17 & 0.00 & 0.00 & 0.02 & 0.00 \\
\hline H-12 & -6.06 & 0.21 & 0.38 & 0.11 & 0.06 & 0.11 & 0.14 \\
\hline $\mathrm{H}-13$ & -6.06 & 0.21 & 0.37 & 0.11 & 0.06 & 0.11 & 0.14 \\
\hline H-14 & -6.30 & 0.21 & 0.16 & 0.03 & 0.12 & 0.46 & 0.02 \\
\hline H-15 & -6.30 & 0.21 & 0.16 & 0.03 & 0.12 & 0.47 & 0.02 \\
\hline H-16 & -6.80 & 0.00 & 0.00 & 0.00 & 0.00 & 1.00 & 0.00 \\
\hline H-17 & -6.80 & 0.00 & 0.00 & 0.00 & 0.00 & 1.00 & 0.00 \\
\hline H-18 & -6.80 & 0.00 & 0.00 & 0.00 & 0.00 & 1.00 & 0.00 \\
\hline H-19 & -6.80 & 0.00 & 0.00 & 0.00 & 0.00 & 1.00 & 0.00 \\
\hline H-20 & -6.85 & 0.00 & 0.00 & 0.00 & 0.00 & 0.98 & 0.00 \\
\hline
\end{tabular}


(Continued)

\begin{tabular}{llllllll} 
H-21 & -6.85 & 0.00 & 0.00 & 0.00 & 0.00 & 0.99 & 0.00 \\
H-22 & -6.86 & 0.00 & 0.00 & 0.00 & 0.00 & 0.99 & 0.00 \\
H-23 & -6.86 & 0.00 & 0.00 & 0.00 & 0.00 & 0.99 & 0.00 \\
H-24 & -6.94 & 0.02 & 0.11 & 0.03 & 0.00 & 0.81 & 0.03 \\
H-25 & -6.94 & 0.01 & 0.10 & 0.03 & 0.00 & 0.82 & 0.03 \\
H-26 & -6.99 & 0.01 & 0.00 & 0.00 & 0.00 & 0.97 & 0.00 \\
H-27 & -6.99 & 0.01 & 0.00 & 0.00 & 0.00 & 0.97 & 0.00 \\
H-28 & -7.05 & 0.03 & 0.13 & 0.02 & 0.01 & 0.78 & 0.03 \\
H-29 & -7.06 & 0.03 & 0.13 & 0.00 & 0.01 & 0.79 & 0.04 \\
H-30 & -7.09 & 0.03 & 0.22 & 0.09 & 0.02 & 0.58 & 0.07 \\
H-31 & -7.09 & 0.03 & 0.22 & 0.09 & 0.02 & 0.60 & 0.06 \\
H-32 & -7.13 & 0.00 & 0.04 & 0.00 & 0.00 & 0.93 & 0.02 \\
H-33 & -7.13 & 0.00 & 0.04 & 0.00 & 0.00 & 0.93 & 0.02 \\
H-34 & -7.14 & 0.00 & 0.02 & 0.00 & 0.00 & 0.95 & 0.00 \\
H-35 & -7.14 & 0.00 & 0.03 & 0.01 & 0.00 & 0.94 & 0.00 \\
H-36 & -7.17 & 0.00 & 0.08 & 0.02 & 0.00 & 0.87 & 0.01 \\
H-37 & -7.17 & 0.00 & 0.07 & 0.01 & 0.00 & 0.88 & 0.02 \\
H-38 & -7.22 & 0.02 & 0.07 & 0.03 & 0.00 & 0.87 & 0.00 \\
H-39 & -7.23 & 0.02 & 0.08 & 0.01 & 0.00 & 0.86 & 0.00 \\
H-40 & -7.46 & 0.02 & 0.33 & 0.34 & 0.03 & 0.25 & 0.03 \\
H-41 & -7.58 & 0.03 & 0.49 & 0.02 & 0.07 & 0.00 & 0.39 \\
H-42 & -7.61 & 0.00 & 0.00 & 0.00 & 0.00 & 0.00 & 0.98 \\
H-43 & -7.61 & 0.00 & 0.02 & 0.00 & 0.00 & 0.02 & 0.96 \\
H-44 & -7.74 & 0.00 & 0.02 & 0.96 & 0.00 & 0.00 & 0.00 \\
H-45 & -7.80 & 0.15 & 0.31 & 0.37 & 0.03 & 0.08 & 0.06 \\
H-46 & -7.81 & 0.11 & 0.44 & 0.25 & 0.05 & 0.13 & 0.03 \\
H-47 & -7.81 & 0.23 & 0.55 & 0.06 & 0.03 & 0.07 & 0.05 \\
H-48 & -7.90 & 0.00 & 0.03 & 0.96 & 0.00 & 0.00 & 0.00 \\
H-49 & -8.00 & 0.00 & 0.94 & 0.00 & 0.00 & 0.00 & 0.04 \\
H-50 & -8.01 & 0.00 & 0.93 & 0.02 & 0.00 & 0.00 & 0.05 \\
\hline & & & & & & & \\
\hline
\end{tabular}


Table S9. Energy and components of molecular orbitals around Fermi energy for the model complex [py- $\left\{\mathrm{Ag}_{2} \mathrm{Br}_{2}\left(\mathrm{PPh}_{3}\right)_{2}\right\}$-bpy- $\left\{\mathrm{Ag}_{2} \mathrm{Br}_{2}\left(\mathrm{PPh}_{3}\right)_{2}\right\}$-py] (2-Br).

\begin{tabular}{|c|c|c|c|c|c|c|c|}
\hline & $\begin{array}{c}\text { Energy } \\
(\mathrm{eV})\end{array}$ & $\mathrm{Br}$ & $\mathrm{Ag}$ & bpy & $\mathrm{P}$ & $\mathrm{Ph}$ & py \\
\hline $\mathrm{L}+10$ & -0.42 & 0.00 & 0.00 & 0.38 & 0.04 & 0.57 & 0.00 \\
\hline $\mathrm{L}+9$ & -0.48 & 0.00 & 0.00 & 0.00 & 0.03 & 0.96 & 0.00 \\
\hline $\mathrm{L}+8$ & -0.49 & 0.00 & 0.00 & 0.07 & 0.03 & 0.90 & 0.00 \\
\hline $\mathrm{L}+7$ & -0.53 & 0.00 & 0.01 & 0.00 & 0.04 & 0.94 & 0.00 \\
\hline $\mathrm{L}+6$ & -0.54 & 0.00 & 0.01 & 0.06 & 0.04 & 0.89 & 0.00 \\
\hline $\mathrm{L}+5$ & -0.57 & 0.00 & 0.00 & 0.00 & 0.01 & 0.00 & 0.98 \\
\hline $\mathrm{L}+4$ & -0.57 & 0.00 & 0.00 & 0.02 & 0.02 & 0.95 & 0.00 \\
\hline $\mathrm{L}+3$ & -1.04 & 0.00 & 0.00 & 0.98 & 0.00 & 0.00 & 0.02 \\
\hline $\mathrm{L}+2$ & -1.10 & 0.00 & 0.00 & 0.00 & 0.00 & 0.00 & 0.98 \\
\hline $\mathrm{L}+1$ & -1.10 & 0.00 & 0.00 & 0.00 & 0.00 & 0.00 & 0.98 \\
\hline LUMO & -2.14 & 0.00 & 0.00 & 0.98 & 0.00 & 0.00 & 0.00 \\
\hline HOMO & -4.87 & 0.52 & 0.24 & 0.00 & 0.15 & 0.09 & 0.00 \\
\hline $\mathrm{H}-1$ & -4.88 & 0.52 & 0.24 & 0.00 & 0.14 & 0.09 & 0.00 \\
\hline $\mathrm{H}-2$ & -5.27 & 0.70 & 0.28 & 0.00 & 0.00 & 0.00 & 0.00 \\
\hline $\mathrm{H}-3$ & -5.27 & 0.70 & 0.28 & 0.00 & 0.00 & 0.00 & 0.00 \\
\hline $\mathrm{H}-4$ & -5.47 & 0.52 & 0.21 & 0.00 & 0.13 & 0.13 & 0.00 \\
\hline H-5 & -5.47 & 0.56 & 0.20 & 0.00 & 0.11 & 0.11 & 0.01 \\
\hline H-6 & -5.48 & 0.68 & 0.18 & 0.04 & 0.03 & 0.03 & 0.04 \\
\hline H-7 & -5.48 & 0.69 & 0.18 & 0.04 & 0.02 & 0.02 & 0.05 \\
\hline H-8 & -5.60 & 0.56 & 0.19 & 0.00 & 0.11 & 0.13 & 0.00 \\
\hline H-9 & -5.60 & 0.52 & 0.20 & 0.00 & 0.12 & 0.15 & 0.00 \\
\hline H-10 & -5.86 & 0.49 & 0.32 & 0.07 & 0.01 & 0.03 & 0.08 \\
\hline H-11 & -5.86 & 0.49 & 0.32 & 0.07 & 0.02 & 0.03 & 0.08 \\
\hline H-12 & -5.99 & 0.51 & 0.27 & 0.05 & 0.04 & 0.07 & 0.06 \\
\hline $\mathrm{H}-13$ & -5.99 & 0.50 & 0.27 & 0.05 & 0.04 & 0.07 & 0.06 \\
\hline H-14 & -6.34 & 0.31 & 0.15 & 0.03 & 0.09 & 0.38 & 0.03 \\
\hline H-15 & -6.34 & 0.31 & 0.15 & 0.03 & 0.09 & 0.39 & 0.03 \\
\hline H-16 & -6.81 & 0.00 & 0.02 & 0.00 & 0.00 & 0.96 & 0.01 \\
\hline H-17 & -6.81 & 0.00 & 0.02 & 0.00 & 0.00 & 0.96 & 0.01 \\
\hline H-18 & -6.82 & 0.00 & 0.00 & 0.00 & 0.00 & 0.99 & 0.00 \\
\hline H-19 & -6.82 & 0.00 & 0.00 & 0.00 & 0.00 & 0.99 & 0.00 \\
\hline H-20 & -6.83 & 0.00 & 0.03 & 0.00 & 0.00 & 0.95 & 0.00 \\
\hline
\end{tabular}


(Continued)

\begin{tabular}{llllllll} 
H-21 & -6.83 & 0.00 & 0.03 & 0.00 & 0.00 & 0.95 & 0.00 \\
H-22 & -6.86 & 0.00 & 0.00 & 0.00 & 0.00 & 0.99 & 0.00 \\
H-23 & -6.86 & 0.00 & 0.00 & 0.00 & 0.00 & 0.99 & 0.00 \\
H-24 & -6.94 & 0.04 & 0.25 & 0.07 & 0.00 & 0.55 & 0.08 \\
H-25 & -6.95 & 0.04 & 0.25 & 0.06 & 0.00 & 0.57 & 0.07 \\
H-26 & -6.98 & 0.02 & 0.08 & 0.03 & 0.00 & 0.87 & 0.00 \\
H-27 & -6.99 & 0.02 & 0.07 & 0.02 & 0.00 & 0.89 & 0.00 \\
H-28 & -7.07 & 0.02 & 0.11 & 0.02 & 0.00 & 0.83 & 0.02 \\
H-29 & -7.07 & 0.02 & 0.11 & 0.01 & 0.00 & 0.82 & 0.03 \\
H-30 & -7.08 & 0.00 & 0.03 & 0.00 & 0.00 & 0.95 & 0.00 \\
H-31 & -7.08 & 0.00 & 0.03 & 0.00 & 0.01 & 0.95 & 0.00 \\
H-32 & -7.10 & 0.00 & 0.04 & 0.02 & 0.01 & 0.92 & 0.00 \\
H-33 & -7.10 & 0.00 & 0.04 & 0.01 & 0.01 & 0.93 & 0.00 \\
H-34 & -7.13 & 0.01 & 0.02 & 0.00 & 0.00 & 0.96 & 0.00 \\
H-35 & -7.13 & 0.01 & 0.01 & 0.00 & 0.00 & 0.96 & 0.00 \\
H-36 & -7.13 & 0.00 & 0.02 & 0.01 & 0.01 & 0.95 & 0.00 \\
H-37 & -7.14 & 0.00 & 0.01 & 0.00 & 0.01 & 0.96 & 0.00 \\
H-38 & -7.19 & 0.03 & 0.10 & 0.04 & 0.00 & 0.80 & 0.00 \\
H-39 & -7.20 & 0.04 & 0.12 & 0.01 & 0.00 & 0.81 & 0.01 \\
H-40 & -7.39 & 0.02 & 0.29 & 0.36 & 0.03 & 0.27 & 0.03 \\
H-41 & -7.53 & 0.04 & 0.45 & 0.01 & 0.06 & 0.00 & 0.43 \\
H-42 & -7.57 & 0.00 & 0.04 & 0.00 & 0.00 & 0.02 & 0.93 \\
H-43 & -7.57 & 0.01 & 0.06 & 0.00 & 0.00 & 0.04 & 0.88 \\
H-44 & -7.65 & 0.21 & 0.57 & 0.04 & 0.02 & 0.08 & 0.07 \\
H-45 & -7.65 & 0.20 & 0.54 & 0.12 & 0.02 & 0.07 & 0.07 \\
H-46 & -7.70 & 0.02 & 0.06 & 0.89 & 0.00 & 0.02 & 0.00 \\
H-47 & -7.73 & 0.06 & 0.29 & 0.43 & 0.04 & 0.14 & 0.04 \\
H-48 & -7.83 & 0.00 & 0.94 & 0.00 & 0.00 & 0.00 & 0.05 \\
H-49 & -7.84 & 0.01 & 0.85 & 0.08 & 0.00 & 0.02 & 0.03 \\
H-50 & -7.86 & 0.00 & 0.04 & 0.93 & 0.00 & 0.02 & 0.00 \\
\hline & & & & & & & \\
\hline
\end{tabular}


Table S10. Energy and components of molecular orbitals around Fermi energy for the model complex [py- $\left\{\mathrm{Ag}_{2} \mathrm{Cl}_{2}\left(\mathrm{PPh}_{3}\right)_{2}\right\}$-bpy- $\left\{\mathrm{Ag}_{2} \mathrm{Cl}_{2}\left(\mathrm{PPh}_{3}\right)_{2}\right\}$-py] (2-Cl).

\begin{tabular}{|c|c|c|c|c|c|c|c|}
\hline & $\begin{array}{c}\text { Energy } \\
(\mathrm{eV})\end{array}$ & $\mathrm{Cl}$ & $\mathrm{Ag}$ & bpy & $\mathrm{P}$ & $\mathrm{Ph}$ & py \\
\hline $\mathrm{L}+10$ & -0.58 & 0.00 & 0.00 & 0.02 & 0.08 & 0.89 & 0.00 \\
\hline $\mathrm{L}+9$ & -0.59 & 0.00 & 0.01 & 0.01 & 0.04 & 0.93 & 0.00 \\
\hline $\mathrm{L}+8$ & -0.60 & 0.00 & 0.00 & 0.01 & 0.03 & 0.92 & 0.03 \\
\hline $\mathrm{L}+7$ & -0.61 & 0.00 & 0.00 & 0.02 & 0.04 & 0.90 & 0.04 \\
\hline $\mathrm{L}+6$ & -0.62 & 0.00 & 0.01 & 0.03 & 0.02 & 0.86 & 0.07 \\
\hline $\mathrm{L}+5$ & -0.65 & 0.00 & 0.00 & 0.00 & 0.05 & 0.77 & 0.17 \\
\hline $\mathrm{L}+4$ & -0.66 & 0.00 & 0.00 & 0.09 & 0.05 & 0.84 & 0.02 \\
\hline $\mathrm{L}+3$ & -0.89 & 0.00 & 0.00 & 0.90 & 0.00 & 0.10 & 0.00 \\
\hline $\mathrm{L}+2$ & -1.00 & 0.00 & 0.00 & 0.00 & 0.00 & 0.03 & 0.96 \\
\hline $\mathrm{L}+1$ & -1.01 & 0.00 & 0.00 & 0.00 & 0.00 & 0.01 & 0.98 \\
\hline LUMO & -1.96 & 0.00 & 0.00 & 0.98 & 0.00 & 0.00 & 0.00 \\
\hline HOMO & -4.92 & 0.56 & 0.28 & 0.00 & 0.10 & 0.06 & 0.00 \\
\hline H-1 & -5.02 & 0.43 & 0.29 & 0.00 & 0.16 & 0.11 & 0.00 \\
\hline $\mathrm{H}-2$ & -5.15 & 0.57 & 0.27 & 0.00 & 0.10 & 0.06 & 0.00 \\
\hline H-3 & -5.24 & 0.69 & 0.28 & 0.00 & 0.00 & 0.00 & 0.00 \\
\hline $\mathrm{H}-4$ & -5.39 & 0.58 & 0.28 & 0.05 & 0.00 & 0.00 & 0.08 \\
\hline H-5 & -5.45 & 0.41 & 0.31 & 0.03 & 0.12 & 0.11 & 0.01 \\
\hline H-6 & -5.47 & 0.82 & 0.15 & 0.01 & 0.00 & 0.00 & 0.01 \\
\hline H-7 & -5.50 & 0.58 & 0.24 & 0.05 & 0.03 & 0.03 & 0.07 \\
\hline H-8 & -5.61 & 0.58 & 0.20 & 0.00 & 0.10 & 0.11 & 0.00 \\
\hline H-9 & -5.68 & 0.37 & 0.26 & 0.02 & 0.15 & 0.18 & 0.02 \\
\hline $\mathrm{H}-10$ & -5.80 & 0.33 & 0.34 & 0.03 & 0.12 & 0.16 & 0.03 \\
\hline H-11 & -5.80 & 0.46 & 0.35 & 0.08 & 0.00 & 0.02 & 0.08 \\
\hline $\mathrm{H}-12$ & -5.90 & 0.56 & 0.30 & 0.03 & 0.03 & 0.05 & 0.03 \\
\hline $\mathrm{H}-13$ & -5.96 & 0.44 & 0.35 & 0.06 & 0.03 & 0.05 & 0.06 \\
\hline $\mathrm{H}-14$ & -6.25 & 0.38 & 0.19 & 0.04 & 0.08 & 0.27 & 0.04 \\
\hline H-15 & -6.34 & 0.39 & 0.18 & 0.04 & 0.07 & 0.27 & 0.04 \\
\hline H-16 & -6.85 & 0.00 & 0.03 & 0.00 & 0.00 & 0.93 & 0.03 \\
\hline H-17 & -6.88 & 0.01 & 0.06 & 0.02 & 0.00 & 0.89 & 0.01 \\
\hline H-18 & -6.91 & 0.01 & 0.03 & 0.00 & 0.00 & 0.94 & 0.00 \\
\hline H-19 & -6.91 & 0.03 & 0.14 & 0.03 & 0.00 & 0.79 & 0.02 \\
\hline H-20 & -6.91 & 0.00 & 0.00 & 0.00 & 0.00 & 0.99 & 0.00 \\
\hline
\end{tabular}


(Continued)

\begin{tabular}{llllllll} 
H-21 & -6.92 & 0.05 & 0.14 & 0.00 & 0.00 & 0.76 & 0.04 \\
H-22 & -6.93 & 0.04 & 0.11 & 0.04 & 0.00 & 0.80 & 0.00 \\
H-23 & -6.94 & 0.00 & 0.00 & 0.00 & 0.00 & 0.99 & 0.00 \\
H-24 & -6.96 & 0.03 & 0.13 & 0.04 & 0.00 & 0.76 & 0.04 \\
H-25 & -7.03 & 0.09 & 0.27 & 0.03 & 0.00 & 0.57 & 0.04 \\
H-26 & -7.05 & 0.03 & 0.12 & 0.05 & 0.00 & 0.76 & 0.02 \\
H-27 & -7.07 & 0.02 & 0.12 & 0.02 & 0.00 & 0.82 & 0.01 \\
H-28 & -7.08 & 0.00 & 0.03 & 0.00 & 0.00 & 0.94 & 0.00 \\
H-29 & -7.09 & 0.02 & 0.07 & 0.02 & 0.00 & 0.87 & 0.02 \\
H-30 & -7.12 & 0.00 & 0.00 & 0.00 & 0.00 & 0.97 & 0.01 \\
H-31 & -7.13 & 0.00 & 0.01 & 0.00 & 0.00 & 0.97 & 0.00 \\
H-32 & -7.15 & 0.01 & 0.05 & 0.02 & 0.00 & 0.90 & 0.00 \\
H-33 & -7.16 & 0.00 & 0.01 & 0.00 & 0.00 & 0.96 & 0.00 \\
H-34 & -7.18 & 0.01 & 0.05 & 0.01 & 0.01 & 0.90 & 0.01 \\
H-35 & -7.18 & 0.02 & 0.14 & 0.01 & 0.01 & 0.81 & 0.01 \\
H-36 & -7.22 & 0.07 & 0.20 & 0.12 & 0.00 & 0.58 & 0.02 \\
H-37 & -7.22 & 0.02 & 0.04 & 0.02 & 0.00 & 0.91 & 0.00 \\
H-38 & -7.24 & 0.04 & 0.17 & 0.01 & 0.01 & 0.76 & 0.00 \\
H-39 & -7.27 & 0.06 & 0.26 & 0.20 & 0.00 & 0.46 & 0.02 \\
H-40 & -7.30 & 0.03 & 0.16 & 0.21 & 0.00 & 0.58 & 0.01 \\
H-41 & -7.37 & 0.00 & 0.15 & 0.00 & 0.01 & 0.82 & 0.01 \\
H-42 & -7.37 & 0.10 & 0.45 & 0.02 & 0.02 & 0.38 & 0.04 \\
H-43 & -7.44 & 0.00 & 0.02 & 0.00 & 0.00 & 0.02 & 0.96 \\
H-44 & -7.46 & 0.01 & 0.41 & 0.00 & 0.00 & 0.09 & 0.47 \\
H-45 & -7.46 & 0.01 & 0.39 & 0.02 & 0.00 & 0.07 & 0.50 \\
H-46 & -7.54 & 0.05 & 0.55 & 0.10 & 0.03 & 0.21 & 0.06 \\
H-47 & -7.55 & 0.04 & 0.50 & 0.27 & 0.01 & 0.09 & 0.08 \\
H-48 & -7.57 & 0.03 & 0.43 & 0.35 & 0.02 & 0.13 & 0.03 \\
H-49 & -7.58 & 0.02 & 0.47 & 0.37 & 0.01 & 0.10 & 0.02 \\
H-50 & -7.68 & 0.01 & 0.12 & 0.81 & 0.00 & 0.03 & 0.01 \\
\hline & & & & & & & \\
\hline
\end{tabular}


Table S11. Energy and components of molecular orbitals around Fermi energy for the model complex $\left[\left\{\left(\mathrm{NH}_{3}\right)\left(\mathrm{PH}_{3}\right)\left(\mathrm{Ag}_{2} \mathrm{I}_{2}\right)\left(\mathrm{PPh}_{3}\right)\right\}\right.$-bpy- $\left.\left\{\left(\mathrm{PPh}_{3}\right)\left(\mathrm{Ag}_{2} \mathrm{I}_{2}\right)\left(\mathrm{NH}_{3}\right)\left(\mathrm{PH}_{3}\right)\right\}\right](3-\mathrm{I})$.

\begin{tabular}{|c|c|c|c|c|c|c|c|c|}
\hline & $\begin{array}{c}\text { Energy } \\
(\mathrm{eV})\end{array}$ & I & $\mathrm{Ag}$ & bpy & $\mathrm{P}(\mathrm{Ph})$ & $\mathrm{Ph}$ & $\mathrm{PH}_{3}$ & $\mathrm{NH}_{3}$ \\
\hline$L+10$ & -0.41 & 0.00 & 0.20 & 0.00 & 0.00 & 0.74 & 0.01 & 0.04 \\
\hline $\mathrm{L}+9$ & -0.41 & 0.00 & 0.20 & 0.00 & 0.00 & 0.74 & 0.01 & 0.04 \\
\hline $\mathrm{L}+8$ & -0.46 & 0.00 & 0.25 & 0.00 & 0.05 & 0.65 & 0.01 & 0.04 \\
\hline $\mathrm{L}+7$ & -0.46 & 0.00 & 0.24 & 0.00 & 0.05 & 0.65 & 0.01 & 0.04 \\
\hline $\mathrm{L}+6$ & -0.50 & 0.00 & 0.02 & 0.91 & 0.00 & 0.07 & 0.00 & 0.00 \\
\hline $\mathrm{L}+5$ & -0.58 & 0.00 & 0.31 & 0.00 & 0.01 & 0.57 & 0.01 & 0.08 \\
\hline $\mathrm{L}+4$ & -0.58 & 0.00 & 0.30 & 0.03 & 0.02 & 0.56 & 0.01 & 0.08 \\
\hline $\mathrm{L}+3$ & -0.67 & 0.00 & 0.00 & 0.00 & 0.07 & 0.91 & 0.00 & 0.00 \\
\hline $\mathrm{L}+2$ & -0.68 & 0.00 & 0.01 & 0.05 & 0.06 & 0.88 & 0.00 & 0.00 \\
\hline $\mathrm{L}+1$ & -1.11 & 0.00 & 0.00 & 0.99 & 0.00 & 0.01 & 0.00 & 0.00 \\
\hline LUMO & -2.19 & 0.00 & 0.00 & 0.99 & 0.00 & 0.00 & 0.00 & 0.00 \\
\hline HOMO & -4.86 & 0.63 & 0.21 & 0.00 & 0.07 & 0.04 & 0.03 & 0.01 \\
\hline $\mathrm{H}-1$ & -4.86 & 0.63 & 0.21 & 0.00 & 0.07 & 0.04 & 0.03 & 0.01 \\
\hline $\mathrm{H}-2$ & -5.18 & 0.73 & 0.25 & 0.00 & 0.00 & 0.00 & 0.00 & 0.00 \\
\hline H-3 & -5.18 & 0.73 & 0.25 & 0.00 & 0.00 & 0.00 & 0.00 & 0.00 \\
\hline $\mathrm{H}-4$ & -5.29 & 0.69 & 0.17 & 0.00 & 0.03 & 0.03 & 0.00 & 0.06 \\
\hline H-5 & -5.29 & 0.69 & 0.17 & 0.01 & 0.03 & 0.02 & 0.00 & 0.06 \\
\hline H-6 & -5.37 & 0.92 & 0.06 & 0.00 & 0.00 & 0.00 & 0.00 & 0.00 \\
\hline H-7 & -5.37 & 0.92 & 0.06 & 0.00 & 0.00 & 0.00 & 0.00 & 0.00 \\
\hline H-8 & -5.65 & 0.47 & 0.28 & 0.04 & 0.07 & 0.08 & 0.06 & 0.02 \\
\hline H-9 & -5.67 & 0.46 & 0.28 & 0.03 & 0.07 & 0.08 & 0.06 & 0.02 \\
\hline H-10 & -5.79 & 0.81 & 0.17 & 0.00 & 0.00 & 0.01 & 0.00 & 0.00 \\
\hline $\mathrm{H}-11$ & -5.79 & 0.81 & 0.17 & 0.00 & 0.00 & 0.01 & 0.00 & 0.00 \\
\hline $\mathrm{H}-12$ & -6.06 & 0.10 & 0.39 & 0.11 & 0.09 & 0.17 & 0.07 & 0.07 \\
\hline $\mathrm{H}-13$ & -6.06 & 0.11 & 0.40 & 0.11 & 0.09 & 0.16 & 0.06 & 0.07 \\
\hline H-14 & -6.67 & 0.18 & 0.33 & 0.03 & 0.01 & 0.18 & 0.18 & 0.08 \\
\hline H-15 & -6.67 & 0.19 & 0.33 & 0.03 & 0.01 & 0.18 & 0.18 & 0.08 \\
\hline H-16 & -6.87 & 0.00 & 0.00 & 0.00 & 0.00 & 1.00 & 0.00 & 0.00 \\
\hline $\mathrm{H}-17$ & -6.87 & 0.00 & 0.00 & 0.00 & 0.00 & 1.00 & 0.00 & 0.00 \\
\hline H-18 & -6.97 & 0.00 & 0.02 & 0.01 & 0.00 & 0.95 & 0.00 & 0.00 \\
\hline H-19 & -6.97 & 0.00 & 0.02 & 0.00 & 0.00 & 0.96 & 0.00 & 0.00 \\
\hline H-20 & -7.05 & 0.02 & 0.10 & 0.04 & 0.00 & 0.82 & 0.00 & 0.00 \\
\hline
\end{tabular}


(Continued)

\begin{tabular}{lllllllll} 
H-21 & -7.05 & 0.02 & 0.10 & 0.03 & 0.00 & 0.84 & 0.00 & 0.00 \\
H-22 & -7.16 & 0.02 & 0.16 & 0.10 & 0.01 & 0.70 & 0.00 & 0.00 \\
H-23 & -7.16 & 0.02 & 0.17 & 0.09 & 0.01 & 0.70 & 0.00 & 0.00 \\
H-24 & -7.21 & 0.00 & 0.08 & 0.03 & 0.00 & 0.88 & 0.00 & 0.00 \\
H-25 & -7.21 & 0.01 & 0.10 & 0.03 & 0.00 & 0.85 & 0.00 & 0.00 \\
H-26 & -7.28 & 0.00 & 0.04 & 0.00 & 0.00 & 0.94 & 0.00 & 0.00 \\
H-27 & -7.28 & 0.00 & 0.03 & 0.00 & 0.00 & 0.95 & 0.00 & 0.00 \\
H-28 & -7.45 & 0.02 & 0.37 & 0.43 & 0.01 & 0.12 & 0.04 & 0.00 \\
H-29 & -7.61 & 0.06 & 0.60 & 0.02 & 0.04 & 0.21 & 0.07 & 0.02 \\
H-30 & -7.73 & 0.00 & 0.01 & 0.98 & 0.00 & 0.00 & 0.00 & 0.00 \\
H-31 & -7.83 & 0.15 & 0.35 & 0.39 & 0.01 & 0.03 & 0.04 & 0.02 \\
H-32 & -7.85 & 0.13 & 0.48 & 0.21 & 0.05 & 0.12 & 0.00 & 0.00 \\
H-33 & -7.85 & 0.25 & 0.60 & 0.05 & 0.02 & 0.05 & 0.01 & 0.02 \\
H-34 & -7.89 & 0.01 & 0.04 & 0.94 & 0.00 & 0.00 & 0.00 & 0.00 \\
H-35 & -8.03 & 0.00 & 0.96 & 0.00 & 0.00 & 0.01 & 0.00 & 0.01 \\
H-36 & -8.03 & 0.00 & 0.95 & 0.01 & 0.00 & 0.00 & 0.00 & 0.01 \\
H-37 & -8.15 & 0.05 & 0.93 & 0.00 & 0.00 & 0.00 & 0.00 & 0.00 \\
H-38 & -8.15 & 0.05 & 0.93 & 0.00 & 0.00 & 0.00 & 0.00 & 0.00 \\
H-39 & -8.24 & 0.06 & 0.87 & 0.01 & 0.00 & 0.04 & 0.00 & 0.00 \\
H-40 & -8.25 & 0.06 & 0.86 & 0.02 & 0.00 & 0.04 & 0.00 & 0.00 \\
\hline
\end{tabular}


Table S12. Energy and major components of excited states for the model complex $\left[\left\{\left(\mathrm{NH}_{3}\right)\left(\mathrm{PH}_{3}\right)\left(\mathrm{Ag}_{2} \mathrm{I}_{2}\right)\left(\mathrm{PPh}_{3}\right)\right\}\right.$-bpy- $\left.\left\{\left(\mathrm{PPh}_{3}\right)\left(\mathrm{Ag}_{2} \mathrm{I}_{2}\right)\left(\mathrm{NH}_{3}\right)\left(\mathrm{PH}_{3}\right)\right\}\right](\mathbf{3}-\mathrm{I})$.

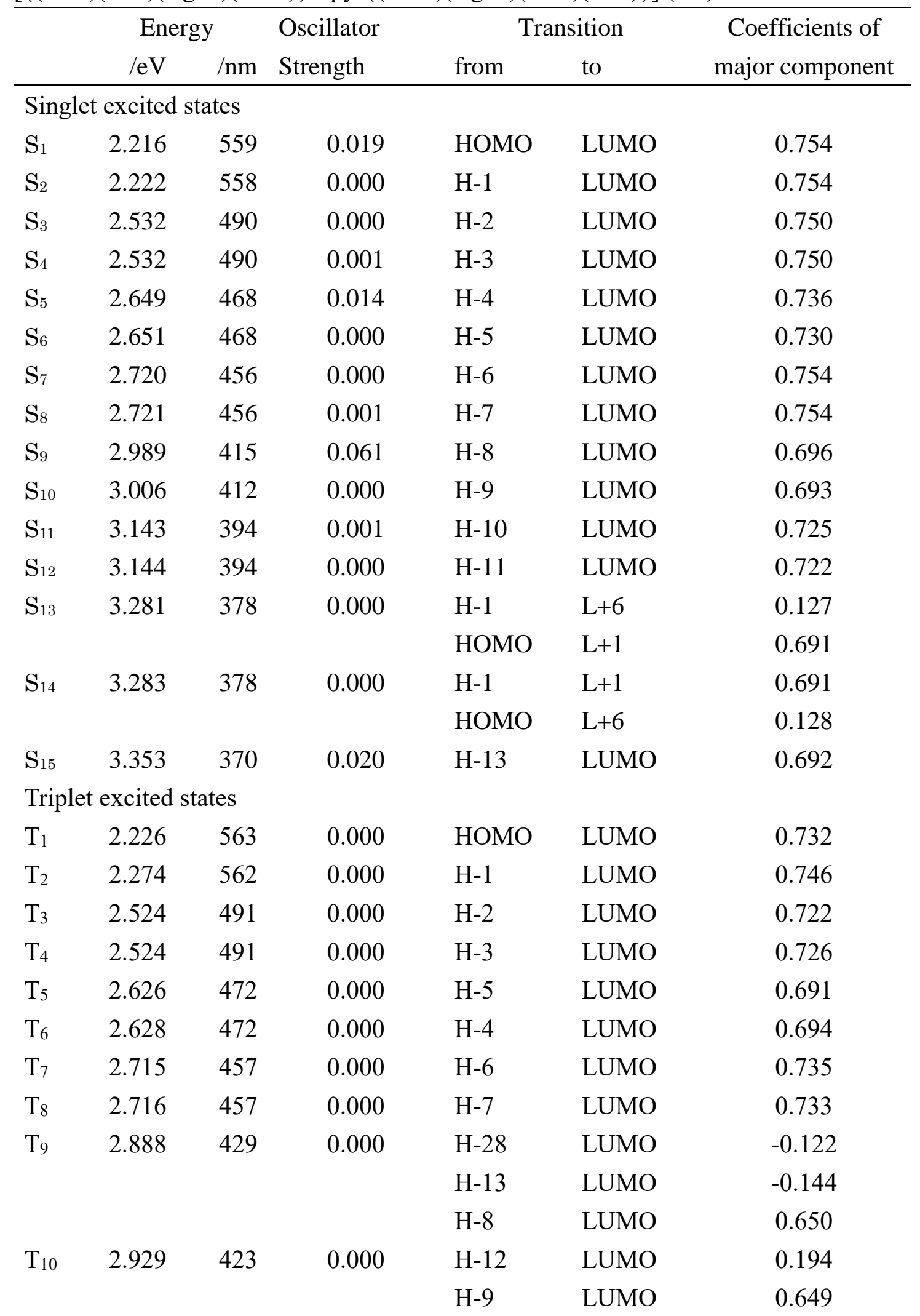


(Continued)

\begin{tabular}{|c|c|c|c|c|c|c|}
\hline & & & & $\mathrm{H}-5$ & LUMO & 0.160 \\
\hline \multirow[t]{3}{*}{$\mathrm{T}_{11}$} & 3.130 & 396 & 0.000 & H-13 & LUMO & -0.189 \\
\hline & & & & H-1 & LUMO & 0.660 \\
\hline & & & & H-8 & LUMO & -0.184 \\
\hline \multirow[t]{3}{*}{$\mathrm{T}_{12}$} & 3.136 & 395 & 0.000 & H-12 & LUMO & 0.172 \\
\hline & & & & $\mathrm{H}-11$ & LUMO & 0.688 \\
\hline & & & & $\mathrm{H}-9$ & LUMO & -0.136 \\
\hline \multirow[t]{5}{*}{$\mathrm{T}_{13}$} & 3.223 & 385 & 0.000 & H-28 & LUMO & -0.124 \\
\hline & & & & H-22 & LUMO & 0.120 \\
\hline & & & & H-13 & LUMO & 0.595 \\
\hline & & & & H-1 & LUMO & 0.238 \\
\hline & & & & H-8 & LUMO & 0.154 \\
\hline \multirow[t]{3}{*}{$\mathrm{T}_{14}$} & 3.255 & 39 & 0.000 & H-12 & LUMO & 0.627 \\
\hline & & & & H-11 & LUMO & -0.156 \\
\hline & & & & H-9 & LUMO & -0.279 \\
\hline \multirow[t]{2}{*}{$\mathrm{T}_{15}$} & 3.277 & 378 & 0.000 & H-1 & $\mathrm{L}+6$ & 0.126 \\
\hline & & & & HOMO & $\mathrm{L}+1$ & 0.689 \\
\hline
\end{tabular}


Table S13. Energy and components of molecular orbitals around Fermi energy for the model complex $\left[\left\{\left(\mathrm{NH}_{3}\right)\left(\mathrm{PH}_{3}\right)\left(\mathrm{Ag}_{2} \mathrm{Br}_{2}\right)\left(\mathrm{PPh}_{3}\right)\right\}\right.$-bpy- $\left.\left\{\left(\mathrm{PPh}_{3}\right)\left(\mathrm{Ag}_{2} \mathrm{Br}_{2}\right)\left(\mathrm{NH}_{3}\right)\left(\mathrm{PH}_{3}\right)\right\}\right](3-\mathrm{Br})$.

\begin{tabular}{|c|c|c|c|c|c|c|c|c|}
\hline & $\begin{array}{c}\text { Energy } \\
(\mathrm{eV})\end{array}$ & $\mathrm{Br}$ & $\mathrm{Ag}$ & bpy & $\mathrm{P}(\mathrm{Ph})$ & $\mathrm{Ph}$ & $\mathrm{PH}_{3}$ & $\mathrm{NH}_{3}$ \\
\hline $\mathrm{L}+10$ & -0.38 & 0.00 & 0.34 & 0.22 & 0.02 & 0.34 & 0.02 & 0.06 \\
\hline $\mathrm{L}+9$ & -0.38 & 0.00 & 0.44 & 0.00 & 0.00 & 0.44 & 0.03 & 0.08 \\
\hline $\mathrm{L}+8$ & -0.39 & 0.00 & 0.11 & 0.66 & 0.00 & 0.20 & 0.00 & 0.02 \\
\hline $\mathrm{L}+7$ & -0.45 & 0.00 & 0.07 & 0.00 & 0.07 & 0.84 & 0.00 & 0.00 \\
\hline $\mathrm{L}+6$ & -0.46 & 0.00 & 0.07 & 0.05 & 0.07 & 0.80 & 0.00 & 0.01 \\
\hline $\mathrm{L}+5$ & -0.54 & 0.00 & 0.25 & 0.00 & 0.02 & 0.65 & 0.01 & 0.06 \\
\hline $\mathrm{L}+4$ & -0.54 & 0.00 & 0.24 & 0.00 & 0.02 & 0.65 & 0.01 & 0.06 \\
\hline $\mathrm{L}+3$ & -0.60 & 0.00 & 0.02 & 0.00 & 0.05 & 0.92 & 0.00 & 0.00 \\
\hline $\mathrm{L}+2$ & -0.61 & 0.00 & 0.03 & 0.03 & 0.04 & 0.89 & 0.00 & 0.00 \\
\hline $\mathrm{L}+1$ & -1.01 & 0.00 & 0.00 & 0.98 & 0.00 & 0.01 & 0.00 & 0.00 \\
\hline LUMO & -2.14 & 0.00 & 0.00 & 0.98 & 0.00 & 0.00 & 0.00 & 0.00 \\
\hline HOMO & -4.95 & 0.57 & 0.25 & 0.00 & 0.08 & 0.05 & 0.03 & 0.01 \\
\hline $\mathrm{H}-1$ & -4.95 & 0.57 & 0.25 & 0.00 & 0.08 & 0.05 & 0.03 & 0.01 \\
\hline H-2 & -5.25 & 0.65 & 0.28 & 0.00 & 0.00 & 0.00 & 0.00 & 0.04 \\
\hline H-3 & -5.25 & 0.65 & 0.29 & 0.00 & 0.00 & 0.00 & 0.00 & 0.04 \\
\hline $\mathrm{H}-4$ & -5.40 & 0.62 & 0.23 & 0.00 & 0.04 & 0.03 & 0.01 & 0.07 \\
\hline H-5 & -5.40 & 0.62 & 0.23 & 0.00 & 0.04 & 0.03 & 0.01 & 0.06 \\
\hline H-6 & -5.54 & 0.85 & 0.10 & 0.00 & 0.01 & 0.01 & 0.00 & 0.00 \\
\hline H-7 & -5.54 & 0.85 & 0.10 & 0.00 & 0.01 & 0.01 & 0.00 & 0.00 \\
\hline H-8 & -5.75 & 0.51 & 0.29 & 0.08 & 0.00 & 0.03 & 0.03 & 0.02 \\
\hline H-9 & -5.75 & 0.50 & 0.31 & 0.08 & 0.00 & 0.03 & 0.03 & 0.03 \\
\hline H-10 & -5.87 & 0.49 & 0.29 & 0.04 & 0.04 & 0.06 & 0.05 & 0.00 \\
\hline H-11 & -5.87 & 0.50 & 0.29 & 0.04 & 0.04 & 0.06 & 0.05 & 0.00 \\
\hline H-12 & -6.04 & 0.40 & 0.28 & 0.05 & 0.07 & 0.13 & 0.05 & 0.00 \\
\hline H-13 & -6.04 & 0.39 & 0.28 & 0.04 & 0.07 & 0.14 & 0.05 & 0.00 \\
\hline H-14 & -6.63 & 0.26 & 0.34 & 0.06 & 0.00 & 0.11 & 0.14 & 0.07 \\
\hline H-15 & -6.63 & 0.27 & 0.34 & 0.05 & 0.00 & 0.11 & 0.14 & 0.07 \\
\hline H-16 & -6.89 & 0.00 & 0.00 & 0.00 & 0.00 & 0.99 & 0.00 & 0.00 \\
\hline H-17 & -6.89 & 0.00 & 0.00 & 0.00 & 0.00 & 0.99 & 0.00 & 0.00 \\
\hline H-18 & -6.93 & 0.00 & 0.04 & 0.01 & 0.00 & 0.93 & 0.00 & 0.00 \\
\hline H-19 & -6.94 & 0.00 & 0.04 & 0.01 & 0.00 & 0.94 & 0.00 & 0.00 \\
\hline H-20 & -7.04 & 0.03 & 0.15 & 0.06 & 0.00 & 0.74 & 0.00 & 0.00 \\
\hline
\end{tabular}


(Continued)

\begin{tabular}{lllllllll} 
H-21 & -7.04 & 0.03 & 0.16 & 0.05 & 0.00 & 0.74 & 0.00 & 0.00 \\
H-22 & -7.15 & 0.02 & 0.16 & 0.05 & 0.00 & 0.75 & 0.00 & 0.00 \\
H-23 & -7.15 & 0.02 & 0.17 & 0.05 & 0.00 & 0.74 & 0.00 & 0.00 \\
H-24 & -7.17 & 0.00 & 0.03 & 0.00 & 0.00 & 0.95 & 0.00 & 0.00 \\
H-25 & -7.17 & 0.00 & 0.02 & 0.00 & 0.00 & 0.96 & 0.00 & 0.00 \\
H-26 & -7.24 & 0.00 & 0.01 & 0.00 & 0.00 & 0.96 & 0.00 & 0.00 \\
H-27 & -7.24 & 0.00 & 0.01 & 0.00 & 0.00 & 0.97 & 0.00 & 0.00 \\
H-28 & -7.38 & 0.03 & 0.33 & 0.46 & 0.00 & 0.13 & 0.04 & 0.00 \\
H-29 & -7.55 & 0.09 & 0.58 & 0.00 & 0.03 & 0.20 & 0.06 & 0.02 \\
H-30 & -7.67 & 0.15 & 0.45 & 0.32 & 0.01 & 0.06 & 0.00 & 0.01 \\
H-31 & -7.67 & 0.25 & 0.62 & 0.05 & 0.00 & 0.03 & 0.01 & 0.02 \\
H-32 & -7.70 & 0.07 & 0.19 & 0.70 & 0.00 & 0.02 & 0.00 & 0.00 \\
H-33 & -7.75 & 0.04 & 0.35 & 0.42 & 0.04 & 0.12 & 0.01 & 0.00 \\
H-34 & -7.84 & 0.01 & 0.96 & 0.00 & 0.00 & 0.00 & 0.00 & 0.01 \\
H-35 & -7.85 & 0.00 & 0.59 & 0.38 & 0.00 & 0.00 & 0.00 & 0.00 \\
H-36 & -7.88 & 0.01 & 0.31 & 0.62 & 0.01 & 0.03 & 0.00 & 0.00 \\
H-37 & -8.01 & 0.07 & 0.90 & 0.00 & 0.00 & 0.01 & 0.00 & 0.00 \\
H-38 & -8.02 & 0.07 & 0.89 & 0.03 & 0.00 & 0.00 & 0.00 & 0.00 \\
H-39 & -8.13 & 0.12 & 0.82 & 0.02 & 0.00 & 0.03 & 0.00 & 0.00 \\
H-40 & -8.13 & 0.11 & 0.81 & 0.03 & 0.00 & 0.03 & 0.00 & 0.00 \\
\hline & & & & & & & &
\end{tabular}


Table S14. Energy and major components of excited states for the model complex $\left[\left\{\left(\mathrm{NH}_{3}\right)\left(\mathrm{PH}_{3}\right)\left(\mathrm{Ag}_{2} \mathrm{Br}_{2}\right)\left(\mathrm{PPh}_{3}\right)\right\}\right.$-bpy- $\left.\left\{\left(\mathrm{PPh}_{3}\right)\left(\mathrm{Ag}_{2} \mathrm{Br}_{2}\right)\left(\mathrm{NH}_{3}\right)\left(\mathrm{PH}_{3}\right)\right\}\right](3-\mathrm{Br})$.

\begin{tabular}{|c|c|c|c|c|c|c|}
\hline & \multicolumn{2}{|c|}{ Energy } & \multirow{2}{*}{$\begin{array}{l}\text { Oscillator } \\
\text { Strength }\end{array}$} & \multicolumn{2}{|c|}{ Transition } & \multirow{2}{*}{$\begin{array}{l}\text { Coefficients of } \\
\text { major component }\end{array}$} \\
\hline & /eV & $/ \mathrm{nm}$ & & from & to & \\
\hline \multicolumn{7}{|c|}{ Singlet excited states } \\
\hline $\mathrm{S}_{1}$ & 2.346 & 528 & 0.024 & HOMO & LUMO & 0.705 \\
\hline $\mathrm{S}_{2}$ & 2.353 & 527 & 0.000 & $\mathrm{H}-1$ & LUMO & 0.705 \\
\hline $\mathrm{S}_{3}$ & 2.644 & 469 & 0.000 & $\mathrm{H}-2$ & LUMO & 0.703 \\
\hline $\mathrm{S}_{4}$ & 2.645 & 469 & 0.001 & $\mathrm{H}-3$ & LUMO & 0.703 \\
\hline $\mathrm{S}_{5}$ & 2.801 & 443 & 0.019 & $\mathrm{H}-4$ & LUMO & 0.702 \\
\hline $\mathrm{S}_{6}$ & 2.804 & 442 & 0.000 & H-5 & LUMO & 0.700 \\
\hline $\mathrm{S}_{7}$ & 2.928 & 423 & 0.000 & H-6 & LUMO & 0.701 \\
\hline $\mathrm{S}_{8}$ & 2.929 & 423 & 0.002 & $\mathrm{H}-7$ & LUMO & 0.702 \\
\hline \multirow[t]{2}{*}{$\mathrm{S}_{9}$} & 3.080 & 403 & 0.031 & $\mathrm{H}-10$ & LUMO & 0.176 \\
\hline & & & & $\mathrm{H}-8$ & LUMO & 0.665 \\
\hline \multirow[t]{2}{*}{$\mathrm{S}_{10}$} & 3.085 & 402 & 0.000 & $\mathrm{H}-11$ & LUMO & -0.174 \\
\hline & & & & $\mathrm{H}-9$ & LUMO & 0.664 \\
\hline \multirow[t]{2}{*}{$\mathrm{S}_{11}$} & 3.258 & 381 & 0.023 & $\mathrm{H}-10$ & LUMO & 0.678 \\
\hline & & & & $\mathrm{H}-8$ & LUMO & -0.189 \\
\hline $\mathrm{S}_{12}$ & 3.264 & 380 & 0.000 & $\mathrm{H}-11$ & LUMO & 0.680 \\
\hline $\mathrm{S}_{13}$ & 3.422 & 362 & 0.027 & H-12 & LUMO & 0.694 \\
\hline $\mathrm{S}_{14}$ & 3.430 & 362 & 0.000 & $\mathrm{H}-13$ & LUMO & 0.694 \\
\hline \multirow[t]{2}{*}{$\mathrm{S}_{15}$} & 3.452 & 359 & 0.000 & $\mathrm{H}-1$ & $\mathrm{~L}+8$ & -0.112 \\
\hline & & & & HOMO & $\mathrm{L}+1$ & 0.690 \\
\hline \multicolumn{7}{|c|}{ Triplet excited states } \\
\hline $\mathrm{T}_{1}$ & 2.328 & 533 & 0.000 & HOMO & LUMO & 0.702 \\
\hline $\mathrm{T}_{2}$ & 2.336 & 531 & 0.000 & $\mathrm{H}-1$ & LUMO & 0.704 \\
\hline $\mathrm{T}_{3}$ & 2.627 & 472 & 0.000 & $\mathrm{H}-2$ & LUMO & 0.692 \\
\hline $\mathrm{T}_{4}$ & 2.629 & 472 & 0.000 & $\mathrm{H}-3$ & LUMO & 0.693 \\
\hline \multirow[t]{2}{*}{$\mathrm{T}_{5}$} & 2.774 & 447 & 0.000 & H-9 & LUMO & 0.159 \\
\hline & & & & $\mathrm{H}-5$ & LUMO & 0.665 \\
\hline \multirow[t]{2}{*}{$\mathrm{T}_{6}$} & 2.775 & 447 & 0.000 & $\mathrm{H}-8$ & LUMO & 0.127 \\
\hline & & & & $\mathrm{H}-4$ & LUMO & 0.673 \\
\hline \multirow[t]{3}{*}{$\mathrm{T}_{7}$} & 2.905 & 427 & 0.000 & $\mathrm{H}-10$ & LUMO & -0.137 \\
\hline & & & & H-8 & LUMO & -0.355 \\
\hline & & & & H-7 & LUMO & 0.536 \\
\hline
\end{tabular}




\section{(Continued)}

\begin{tabular}{|c|c|c|c|c|c|c|}
\hline & & & & $\mathrm{H}-4$ & LUMO & 0.178 \\
\hline \multirow[t]{4}{*}{$\mathrm{T}_{8}$} & 2.906 & 427 & 0.000 & $\mathrm{H}-11$ & LUMO & -0.107 \\
\hline & & & & H-9 & LUMO & 0.261 \\
\hline & & & & H-6 & LUMO & 0.603 \\
\hline & & & & H-5 & LUMO & -0.183 \\
\hline \multirow[t]{4}{*}{$\mathrm{T}_{9}$} & 2.942 & 421 & 0.000 & $\mathrm{H}-28$ & LUMO & 0.114 \\
\hline & & & & $\mathrm{H}-10$ & LUMO & 0.127 \\
\hline & & & & H-8 & LUMO & 0.482 \\
\hline & & & & $\mathrm{H}-7$ & LUMO & 0.441 \\
\hline \multirow[t]{5}{*}{$\mathrm{T}_{10}$} & 2.973 & 417 & 0.000 & $\mathrm{H}-13$ & LUMO & -0.122 \\
\hline & & & & H-11 & LUMO & -0.184 \\
\hline & & & & H-9 & LUMO & 0.526 \\
\hline & & & & H-6 & LUMO & -0.351 \\
\hline & & & & $\mathrm{H}-5$ & LUMO & -0.139 \\
\hline \multirow[t]{5}{*}{$\mathrm{T}_{11}$} & 3.188 & 389 & 0.000 & H-33 & LUMO & 0.112 \\
\hline & & & & H-28 & LUMO & -0.180 \\
\hline & & & & H-12 & LUMO & 0.204 \\
\hline & & & & $\mathrm{H}-10$ & LUMO & 0.573 \\
\hline & & & & H-8 & LUMO & -0.205 \\
\hline \multirow[t]{2}{*}{$\mathrm{T}_{12}$} & 3.240 & 383 & 0.000 & $\mathrm{H}-11$ & LUMO & 0.638 \\
\hline & & & & H-9 & LUMO & 0.278 \\
\hline \multirow[t]{4}{*}{$\mathrm{T}_{13}$} & 3.360 & 369 & 0.000 & H-33 & LUMO & 0.110 \\
\hline & & & & H-28 & LUMO & -0.173 \\
\hline & & & & $\mathrm{H}-12$ & LUMO & 0.567 \\
\hline & & & & $\mathrm{H}-10$ & LUMO & -0.325 \\
\hline \multirow[t]{3}{*}{$\mathrm{T}_{14}$} & 3.397 & 365 & 0.000 & $\mathrm{H}-13$ & LUMO & 0.666 \\
\hline & & & & $\mathrm{H}-11$ & LUMO & -0.154 \\
\hline & & & & H-9 & LUMO & 0.139 \\
\hline \multirow[t]{2}{*}{$\mathrm{T}_{15}$} & 3.447 & 360 & 0.000 & H-1 & $\mathrm{L}+8$ & -0.113 \\
\hline & & & & HOMO & $\mathrm{L}+1$ & 0.688 \\
\hline
\end{tabular}


Table S15. Energy and components of molecular orbitals around Fermi energy for the model complex $\left[\left\{\left(\mathrm{NH}_{3}\right)\left(\mathrm{PH}_{3}\right)\left(\mathrm{Ag}_{2} \mathrm{Cl}_{2}\right)\left(\mathrm{PPh}_{3}\right)\right\}\right.$-bpy- $\left.\left\{\left(\mathrm{PPh}_{3}\right)\left(\mathrm{Ag}_{2} \mathrm{Cl}_{2}\right)\left(\mathrm{NH}_{3}\right)\left(\mathrm{PH}_{3}\right)\right\}\right](\mathbf{3}-\mathbf{C l})$.

\begin{tabular}{|c|c|c|c|c|c|c|c|c|}
\hline & $\begin{array}{c}\text { Energy } \\
(\mathrm{eV})\end{array}$ & $\mathrm{Cl}$ & $\mathrm{Ag}$ & bpy & $\mathrm{P}(\mathrm{Ph})$ & $\mathrm{Ph}$ & $\mathrm{PH}_{3}$ & $\mathrm{NH}_{3}$ \\
\hline $\mathrm{L}+10$ & -0.38 & 0.00 & 0.65 & 0.00 & 0.00 & 0.19 & 0.04 & 0.10 \\
\hline $\mathrm{L}+9$ & -0.42 & 0.00 & 0.02 & 0.85 & 0.00 & 0.12 & 0.00 & 0.00 \\
\hline $\mathrm{L}+8$ & -0.45 & 0.00 & 0.71 & 0.00 & 0.00 & 0.11 & 0.04 & 0.13 \\
\hline $\mathrm{L}+7$ & -0.50 & 0.00 & 0.07 & 0.00 & 0.03 & 0.87 & 0.00 & 0.01 \\
\hline $\mathrm{L}+6$ & -0.51 & 0.00 & 0.03 & 0.03 & 0.07 & 0.87 & 0.00 & 0.00 \\
\hline $\mathrm{L}+5$ & -0.60 & 0.00 & 0.03 & 0.03 & 0.04 & 0.90 & 0.00 & 0.00 \\
\hline $\mathrm{L}+4$ & -0.61 & 0.00 & 0.00 & 0.00 & 0.08 & 0.91 & 0.00 & 0.00 \\
\hline $\mathrm{L}+3$ & -0.61 & 0.00 & 0.04 & 0.04 & 0.02 & 0.89 & 0.00 & 0.00 \\
\hline $\mathrm{L}+2$ & -0.66 & 0.00 & 0.02 & 0.09 & 0.05 & 0.84 & 0.00 & 0.00 \\
\hline $\mathrm{L}+1$ & -0.89 & 0.00 & 0.00 & 0.92 & 0.00 & 0.07 & 0.00 & 0.00 \\
\hline LUMO & -1.98 & 0.00 & 0.00 & 0.99 & 0.00 & 0.00 & 0.00 & 0.00 \\
\hline HOMO & -4.96 & 0.62 & 0.27 & 0.00 & 0.03 & 0.02 & 0.03 & 0.02 \\
\hline $\mathrm{H}-1$ & -5.07 & 0.47 & 0.32 & 0.00 & 0.08 & 0.06 & 0.02 & 0.04 \\
\hline H-2 & -5.13 & 0.43 & 0.37 & 0.00 & 0.06 & 0.04 & 0.00 & 0.09 \\
\hline H-3 & -5.19 & 0.64 & 0.29 & 0.00 & 0.00 & 0.00 & 0.01 & 0.04 \\
\hline H-4 & -5.26 & 0.56 & 0.27 & 0.01 & 0.04 & 0.03 & 0.04 & 0.04 \\
\hline H-5 & -5.38 & 0.52 & 0.28 & 0.00 & 0.04 & 0.04 & 0.03 & 0.08 \\
\hline H-6 & -5.51 & 0.82 & 0.15 & 0.01 & 0.00 & 0.00 & 0.00 & 0.00 \\
\hline H-7 & -5.57 & 0.53 & 0.32 & 0.05 & 0.02 & 0.03 & 0.04 & 0.00 \\
\hline H-8 & -5.64 & 0.78 & 0.16 & 0.03 & 0.00 & 0.00 & 0.00 & 0.02 \\
\hline H-9 & -5.75 & 0.45 & 0.33 & 0.14 & 0.02 & 0.02 & 0.00 & 0.03 \\
\hline $\mathrm{H}-10$ & -5.82 & 0.39 & 0.40 & 0.11 & 0.02 & 0.02 & 0.02 & 0.04 \\
\hline $\mathrm{H}-11$ & -5.86 & 0.64 & 0.29 & 0.00 & 0.01 & 0.02 & 0.03 & 0.00 \\
\hline H-12 & -5.99 & 0.26 & 0.34 & 0.01 & 0.10 & 0.18 & 0.11 & 0.00 \\
\hline H-13 & -6.01 & 0.37 & 0.30 & 0.00 & 0.09 & 0.17 & 0.06 & 0.00 \\
\hline H-14 & -6.46 & 0.39 & 0.33 & 0.04 & 0.00 & 0.07 & 0.13 & 0.05 \\
\hline H-15 & -6.53 & 0.34 & 0.35 & 0.06 & 0.01 & 0.06 & 0.12 & 0.06 \\
\hline H-16 & -6.91 & 0.00 & 0.01 & 0.00 & 0.00 & 0.97 & 0.00 & 0.00 \\
\hline H-17 & -6.92 & 0.00 & 0.01 & 0.00 & 0.00 & 0.98 & 0.00 & 0.00 \\
\hline H-18 & -6.95 & 0.04 & 0.10 & 0.03 & 0.00 & 0.82 & 0.00 & 0.00 \\
\hline H-19 & -6.97 & 0.00 & 0.03 & 0.00 & 0.00 & 0.95 & 0.00 & 0.00 \\
\hline H-20 & -7.04 & 0.06 & 0.27 & 0.06 & 0.00 & 0.59 & 0.01 & 0.00 \\
\hline
\end{tabular}


(Continued)

\begin{tabular}{lllllllll} 
H-21 & -7.05 & 0.10 & 0.34 & 0.08 & 0.00 & 0.47 & 0.00 & 0.00 \\
H-22 & -7.09 & 0.00 & 0.02 & 0.00 & 0.00 & 0.97 & 0.00 & 0.00 \\
H-23 & -7.16 & 0.07 & 0.41 & 0.01 & 0.00 & 0.47 & 0.02 & 0.02 \\
H-24 & -7.17 & 0.00 & 0.00 & 0.00 & 0.00 & 0.99 & 0.00 & 0.00 \\
H-25 & -7.19 & 0.03 & 0.05 & 0.00 & 0.00 & 0.90 & 0.00 & 0.00 \\
H-26 & -7.21 & 0.02 & 0.08 & 0.01 & 0.00 & 0.87 & 0.00 & 0.00 \\
H-27 & -7.22 & 0.02 & 0.15 & 0.07 & 0.00 & 0.75 & 0.00 & 0.00 \\
H-28 & -7.26 & 0.06 & 0.36 & 0.42 & 0.00 & 0.14 & 0.01 & 0.00 \\
H-29 & -7.32 & 0.15 & 0.41 & 0.13 & 0.00 & 0.29 & 0.00 & 0.02 \\
H-30 & -7.33 & 0.08 & 0.29 & 0.01 & 0.00 & 0.59 & 0.01 & 0.01 \\
H-31 & -7.48 & 0.03 & 0.80 & 0.01 & 0.01 & 0.13 & 0.00 & 0.00 \\
H-32 & -7.53 & 0.05 & 0.63 & 0.05 & 0.02 & 0.23 & 0.01 & 0.00 \\
H-33 & -7.55 & 0.00 & 0.04 & 0.93 & 0.00 & 0.02 & 0.00 & 0.00 \\
H-34 & -7.57 & 0.02 & 0.89 & 0.02 & 0.00 & 0.05 & 0.00 & 0.01 \\
H-35 & -7.62 & 0.06 & 0.73 & 0.10 & 0.01 & 0.08 & 0.00 & 0.00 \\
H-36 & -7.69 & 0.00 & 0.02 & 0.97 & 0.00 & 0.00 & 0.00 & 0.00 \\
H-37 & -7.72 & 0.09 & 0.78 & 0.08 & 0.00 & 0.04 & 0.00 & 0.00 \\
H-38 & -7.83 & 0.07 & 0.71 & 0.19 & 0.00 & 0.01 & 0.00 & 0.00 \\
H-39 & -7.94 & 0.28 & 0.62 & 0.06 & 0.00 & 0.02 & 0.01 & 0.00 \\
H-40 & -7.99 & 0.24 & 0.66 & 0.07 & 0.00 & 0.00 & 0.02 & 0.00 \\
\hline & & & & & & & &
\end{tabular}


Table S16. Energy and major components of excited states for the model complex $\left[\left\{\left(\mathrm{NH}_{3}\right)\left(\mathrm{PH}_{3}\right)\left(\mathrm{Ag}_{2} \mathrm{Cl}_{2}\right)\left(\mathrm{PPh}_{3}\right)\right\}\right.$-bpy- $\left.\left\{\left(\mathrm{PPh}_{3}\right)\left(\mathrm{Ag}_{2} \mathrm{Cl}_{2}\right)\left(\mathrm{NH}_{3}\right)\left(\mathrm{PH}_{3}\right)\right\}\right](\mathbf{3}-\mathbf{C l})$.

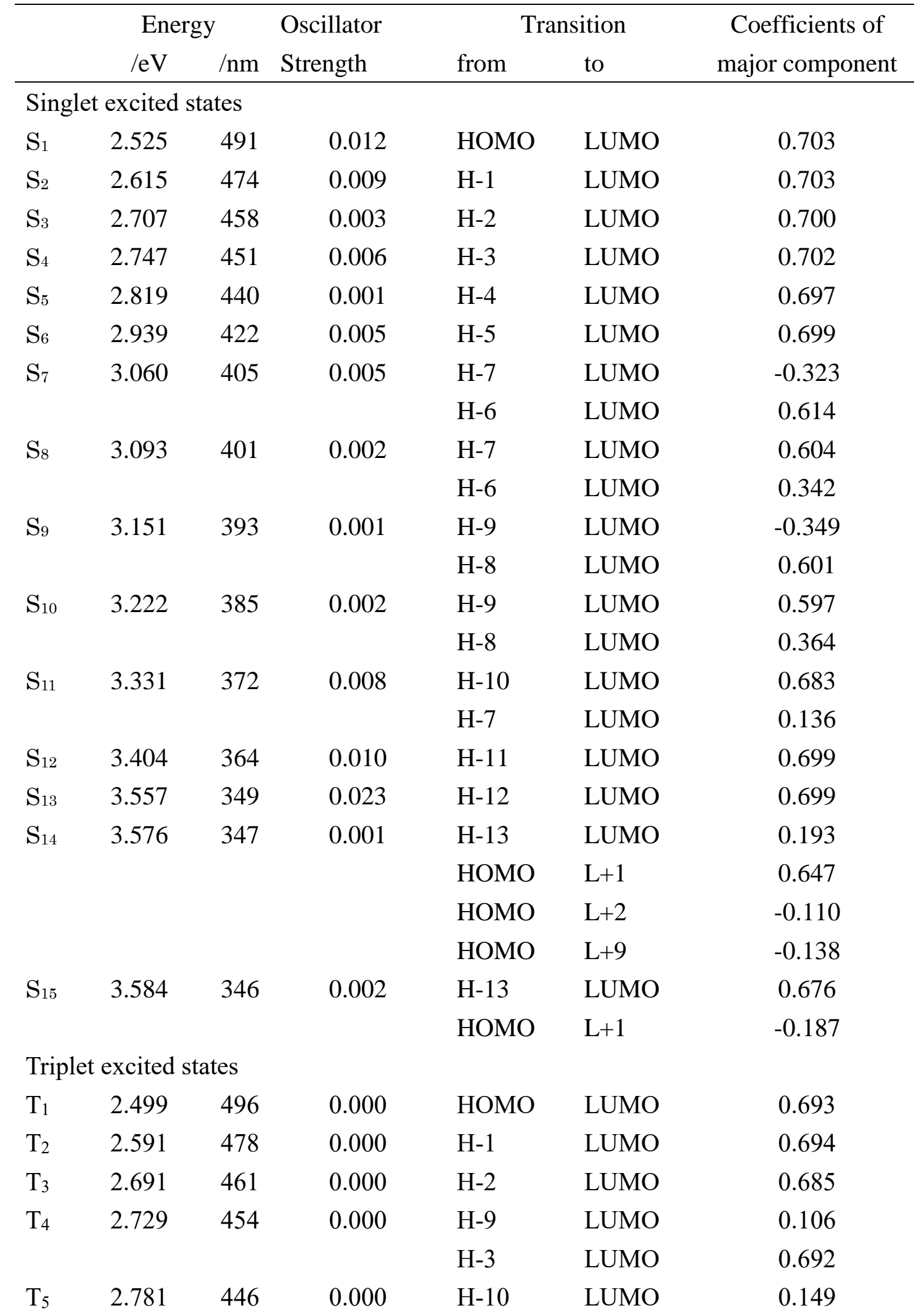


(Continued)

\begin{tabular}{|c|c|c|c|c|c|c|}
\hline & & & & $\mathrm{H}-7$ & LUMO & -0.163 \\
\hline & & & & $\mathrm{H}-4$ & LUMO & 0.647 \\
\hline & & & & $\mathrm{H}-2$ & LUMO & -0.113 \\
\hline \multirow[t]{3}{*}{$\mathrm{T}_{6}$} & 2.899 & 428 & 0.000 & H-9 & LUMO & -0.279 \\
\hline & & & & H-8 & LUMO & 0.137 \\
\hline & & & & $\mathrm{H}-5$ & LUMO & 0.611 \\
\hline \multirow[t]{4}{*}{$\mathrm{T}_{7}$} & 2.981 & 416 & 0.000 & $\mathrm{H}-10$ & LUMO & -0.208 \\
\hline & & & & $\mathrm{H}-7$ & LUMO & 0.490 \\
\hline & & & & H-6 & LUMO & -0.328 \\
\hline & & & & $\mathrm{H}-4$ & LUMO & 0.247 \\
\hline \multirow[t]{3}{*}{$\mathrm{T}_{8}$} & 3.024 & 410 & 0.000 & H-9 & LUMO & 0.457 \\
\hline & & & & H-8 & LUMO & -0.335 \\
\hline & & & & H-5 & LUMO & 0.342 \\
\hline \multirow[t]{2}{*}{$\mathrm{T}_{9}$} & 3.079 & 403 & 0.000 & $\mathrm{H}-7$ & LUMO & 0.341 \\
\hline & & & & H-6 & LUMO & 0.610 \\
\hline \multirow[t]{3}{*}{$\mathrm{T}_{10}$} & 3.196 & 388 & 0.000 & $\mathrm{H}-10$ & LUMO & -0.114 \\
\hline & & & & $\mathrm{H}-9$ & LUMO & 0.376 \\
\hline & & & & $\mathrm{H}-8$ & LUMO & 0.562 \\
\hline \multirow[t]{5}{*}{$\mathrm{T}_{11}$} & 3.232 & 384 & 0.000 & $\mathrm{H}-28$ & LUMO & 0.191 \\
\hline & & & & $\mathrm{H}-11$ & LUMO & -0.134 \\
\hline & & & & $\mathrm{H}-10$ & LUMO & 0.529 \\
\hline & & & & H-8 & LUMO & 0.184 \\
\hline & & & & $\mathrm{H}-7$ & LUMO & 0.225 \\
\hline \multirow[t]{5}{*}{$\mathrm{T}_{12}$} & 3.341 & 371 & 0.000 & $\mathrm{H}-30$ & LUMO & 0.108 \\
\hline & & & & H-28 & LUMO & -0.216 \\
\hline & & & & $\mathrm{H}-11$ & LUMO & 0.522 \\
\hline & & & & $\mathrm{H}-10$ & LUMO & 0.253 \\
\hline & & & & $\mathrm{H}-7$ & LUMO & 0.159 \\
\hline \multirow[t]{7}{*}{$\mathrm{T}_{13}$} & 3.459 & 358 & 0.000 & H-38 & LUMO & 0.108 \\
\hline & & & & $\mathrm{H}-34$ & $\mathrm{~L}+1$ & -0.115 \\
\hline & & & & $\mathrm{H}-30$ & LUMO & -0.122 \\
\hline & & & & H-28 & LUMO & 0.256 \\
\hline & & & & $\mathrm{H}-12$ & LUMO & 0.367 \\
\hline & & & & H-11 & LUMO & 0.408 \\
\hline & & & & $\mathrm{H}-10$ & LUMO & -0.124 \\
\hline
\end{tabular}




\begin{tabular}{|c|c|c|c|c|c|c|}
\hline \multicolumn{7}{|c|}{ (Continued) } \\
\hline $\mathrm{T}_{14}$ & 3.550 & 349 & 0.000 & $\mathrm{H}-2$ & $\mathrm{~L}+1$ & 0.106 \\
\hline \multirow{7}{*}{$\mathrm{T}_{15}$} & \multirow{7}{*}{3.565} & \multirow{7}{*}{348} & \multirow{7}{*}{0.000} & $\mathrm{H}-2$ & $\mathrm{~L}+2$ & -0.156 \\
\hline & & & & HOMO & $\mathrm{L}+1$ & 0.541 \\
\hline & & & & HOMO & $\mathrm{L}+2$ & -0.243 \\
\hline & & & & H-28 & LUMO & 0.115 \\
\hline & & & & $\mathrm{H}-13$ & LUMO & 0.472 \\
\hline & & & & H-12 & LUMO & -0.451 \\
\hline & & & & H-11 & LUMO & 0.112 \\
\hline
\end{tabular}


Table S17. Energy and components of molecular orbitals around Fermi energy for the model complex $\left[\left\{\left(\mathrm{NH}_{3}\right)\left(\mathrm{PH}_{3}\right)\left(\mathrm{Ag}_{2} \mathrm{I}_{2}\right)\left(\mathrm{PPh}_{3}\right)\right\}\right.$-bpy- $\left.\left\{\left(\mathrm{PPh}_{3}\right)\left(\mathrm{Ag}_{2} \mathrm{I}_{2}\right)\left(\mathrm{NH}_{3}\right)\left(\mathrm{PH}_{3}\right)\right\}\right]($ 3-I $)$ in DMSO.

\begin{tabular}{|c|c|c|c|c|c|c|c|c|}
\hline & $\begin{array}{c}\text { Energy } \\
(\mathrm{eV})\end{array}$ & I & $\mathrm{Ag}$ & bpy & $\mathrm{P}(\mathrm{Ph})$ & $\mathrm{Ph}$ & $\mathrm{PH}_{3}$ & $\mathrm{NH}_{3}$ \\
\hline $\mathrm{L}+10$ & -0.43 & 0.00 & 0.17 & 0.03 & 0.00 & 0.76 & 0.00 & 0.02 \\
\hline $\mathrm{L}+9$ & -0.43 & 0.01 & 0.17 & 0.00 & 0.00 & 0.79 & 0.00 & 0.02 \\
\hline $\mathrm{L}+8$ & -0.54 & 0.00 & 0.00 & 0.93 & 0.00 & 0.06 & 0.00 & 0.00 \\
\hline $\mathrm{L}+7$ & -0.64 & 0.00 & 0.03 & 0.00 & 0.04 & 0.91 & 0.00 & 0.00 \\
\hline $\mathrm{L}+6$ & -0.64 & 0.00 & 0.03 & 0.00 & 0.05 & 0.91 & 0.00 & 0.00 \\
\hline $\mathrm{L}+5$ & -0.71 & 0.00 & 0.02 & 0.00 & 0.04 & 0.93 & 0.00 & 0.00 \\
\hline $\mathrm{L}+4$ & -0.71 & 0.00 & 0.02 & 0.00 & 0.04 & 0.93 & 0.00 & 0.00 \\
\hline $\mathrm{L}+3$ & -0.88 & 0.00 & 0.02 & 0.02 & 0.07 & 0.89 & 0.00 & 0.00 \\
\hline $\mathrm{L}+2$ & -0.87 & 0.00 & 0.02 & 0.01 & 0.07 & 0.90 & 0.00 & 0.00 \\
\hline $\mathrm{L}+1$ & -1.13 & 0.00 & 0.00 & 0.97 & 0.00 & 0.03 & 0.00 & 0.00 \\
\hline LUMO & -2.23 & 0.00 & 0.00 & 0.99 & 0.00 & 0.00 & 0.00 & 0.00 \\
\hline HOMO & -5.47 & 0.56 & 0.22 & 0.00 & 0.09 & 0.07 & 0.04 & 0.02 \\
\hline H-1 & -5.47 & 0.56 & 0.22 & 0.00 & 0.09 & 0.06 & 0.04 & 0.02 \\
\hline $\mathrm{H}-2$ & -5.82 & 0.49 & 0.27 & 0.00 & 0.04 & 0.05 & 0.00 & 0.13 \\
\hline $\mathrm{H}-3$ & -5.82 & 0.50 & 0.27 & 0.00 & 0.04 & 0.04 & 0.00 & 0.13 \\
\hline $\mathrm{H}-4$ & -5.92 & 0.68 & 0.26 & 0.00 & 0.02 & 0.02 & 0.00 & 0.01 \\
\hline H-5 & -5.92 & 0.68 & 0.26 & 0.00 & 0.02 & 0.02 & 0.00 & 0.01 \\
\hline H-6 & -6.10 & 0.52 & 0.21 & 0.00 & 0.05 & 0.08 & 0.12 & 0.00 \\
\hline H-7 & -6.11 & 0.55 & 0.20 & 0.00 & 0.05 & 0.08 & 0.11 & 0.00 \\
\hline H-8 & -6.19 & 0.71 & 0.15 & 0.02 & 0.02 & 0.04 & 0.06 & 0.00 \\
\hline H-9 & -6.19 & 0.67 & 0.16 & 0.02 & 0.03 & 0.05 & 0.07 & 0.00 \\
\hline H-10 & -6.38 & 0.29 & 0.35 & 0.16 & 0.03 & 0.07 & 0.03 & 0.08 \\
\hline H-11 & -6.38 & 0.29 & 0.35 & 0.16 & 0.03 & 0.07 & 0.03 & 0.07 \\
\hline $\mathrm{H}-12$ & -6.54 & 0.79 & 0.16 & 0.00 & 0.00 & 0.04 & 0.00 & 0.00 \\
\hline $\mathrm{H}-13$ & -6.54 & 0.79 & 0.16 & 0.00 & 0.00 & 0.04 & 0.00 & 0.00 \\
\hline H-14 & -7.00 & 0.30 & 0.21 & 0.03 & 0.00 & 0.28 & 0.13 & 0.05 \\
\hline H-15 & -7.01 & 0.30 & 0.21 & 0.02 & 0.00 & 0.29 & 0.13 & 0.04 \\
\hline H-16 & -7.10 & 0.00 & 0.00 & 0.00 & 0.00 & 0.98 & 0.00 & 0.00 \\
\hline H-17 & -7.10 & 0.00 & 0.00 & 0.00 & 0.00 & 0.98 & 0.00 & 0.00 \\
\hline H-18 & -7.16 & 0.00 & 0.00 & 0.00 & 0.00 & 0.98 & 0.00 & 0.00 \\
\hline H-19 & -7.16 & 0.00 & 0.00 & 0.00 & 0.00 & 0.98 & 0.00 & 0.00 \\
\hline $\mathrm{H}-20$ & -7.27 & 0.02 & 0.04 & 0.01 & 0.00 & 0.91 & 0.00 & 0.00 \\
\hline
\end{tabular}


(Continued)

\begin{tabular}{lllllllll} 
H-21 & -7.27 & 0.02 & 0.03 & 0.00 & 0.00 & 0.92 & 0.00 & 0.00 \\
H-22 & -7.38 & 0.02 & 0.05 & 0.06 & 0.00 & 0.86 & 0.00 & 0.00 \\
H-23 & -7.38 & 0.01 & 0.04 & 0.03 & 0.00 & 0.92 & 0.00 & 0.00 \\
H-24 & -7.43 & 0.04 & 0.13 & 0.11 & 0.02 & 0.69 & 0.00 & 0.01 \\
H-25 & -7.44 & 0.05 & 0.15 & 0.08 & 0.02 & 0.69 & 0.00 & 0.01 \\
H-26 & -7.50 & 0.02 & 0.12 & 0.05 & 0.00 & 0.79 & 0.00 & 0.00 \\
H-27 & -7.50 & 0.03 & 0.18 & 0.07 & 0.00 & 0.70 & 0.00 & 0.01 \\
H-28 & -7.61 & 0.02 & 0.21 & 0.72 & 0.00 & 0.03 & 0.02 & 0.00 \\
H-29 & -7.76 & 0.00 & 0.00 & 0.99 & 0.00 & 0.00 & 0.00 & 0.00 \\
H-30 & -7.90 & 0.00 & 0.00 & 0.99 & 0.00 & 0.00 & 0.00 & 0.00 \\
H-31 & -7.94 & 0.08 & 0.57 & 0.02 & 0.06 & 0.20 & 0.06 & 0.01 \\
H-32 & -8.06 & 0.07 & 0.41 & 0.22 & 0.07 & 0.19 & 0.04 & 0.00 \\
H-33 & -8.36 & 0.29 & 0.59 & 0.05 & 0.01 & 0.02 & 0.02 & 0.02 \\
H-34 & -8.36 & 0.27 & 0.62 & 0.03 & 0.01 & 0.02 & 0.02 & 0.02 \\
H-35 & -8.39 & 0.02 & 0.95 & 0.00 & 0.00 & 0.00 & 0.00 & 0.02 \\
H-36 & -8.39 & 0.02 & 0.95 & 0.00 & 0.00 & 0.00 & 0.00 & 0.02 \\
H-37 & -8.59 & 0.09 & 0.88 & 0.01 & 0.00 & 0.00 & 0.00 & 0.00 \\
H-38 & -8.60 & 0.09 & 0.89 & 0.00 & 0.00 & 0.00 & 0.00 & 0.00 \\
H-39 & -8.67 & 0.07 & 0.83 & 0.01 & 0.00 & 0.07 & 0.00 & 0.00 \\
H-40 & -8.68 & 0.07 & 0.83 & 0.02 & 0.00 & 0.07 & 0.00 & 0.00 \\
\hline & & & & & & & &
\end{tabular}


Table S18. Energy and major components of excited states for the model complex $\left[\left\{\left(\mathrm{NH}_{3}\right)\left(\mathrm{PH}_{3}\right)\left(\mathrm{Ag}_{2} \mathrm{I}_{2}\right)\left(\mathrm{PPh}_{3}\right)\right\}\right.$-bpy- $\left.\left\{\left(\mathrm{PPh}_{3}\right)\left(\mathrm{Ag}_{2} \mathrm{I}_{2}\right)\left(\mathrm{NH}_{3}\right)\left(\mathrm{PH}_{3}\right)\right\}\right](3-\mathrm{I})$ in DMSO.

\begin{tabular}{|c|c|c|c|c|c|c|}
\hline & \multicolumn{2}{|c|}{ Energy } & \multirow{2}{*}{$\begin{array}{l}\text { Oscillator } \\
\text { Strength }\end{array}$} & \multicolumn{2}{|c|}{ Transition } & \multirow{2}{*}{$\begin{array}{l}\text { Coefficients of } \\
\text { major component }\end{array}$} \\
\hline & leV & $/ \mathrm{nm}$ & & from & to & \\
\hline \multicolumn{7}{|c|}{ Singlet excited states } \\
\hline $\mathrm{S}_{1}$ & 2.789 & 445 & 0.024 & HOMO & LUMO & 0.705 \\
\hline $\mathrm{S}_{2}$ & 2.794 & 444 & 0.000 & $\mathrm{H}-1$ & LUMO & 0.705 \\
\hline $\mathrm{S}_{3}$ & 3.147 & 394 & 0.024 & $\mathrm{H}-2$ & LUMO & 0.702 \\
\hline $\mathrm{S}_{4}$ & 3.150 & 394 & 0.000 & $\mathrm{H}-3$ & LUMO & 0.700 \\
\hline $\mathrm{S}_{5}$ & 3.235 & 383 & 0.015 & $\mathrm{H}-4$ & LUMO & 0.703 \\
\hline $\mathrm{S}_{6}$ & 3.238 & 383 & 0.000 & $\mathrm{H}-5$ & LUMO & 0.702 \\
\hline $\mathrm{S}_{7}$ & 3.422 & 362 & 0.025 & H-6 & LUMO & 0.700 \\
\hline \multirow[t]{2}{*}{$\mathrm{S}_{8}$} & 3.428 & 362 & 0.000 & $\mathrm{H}-9$ & LUMO & -0.108 \\
\hline & & & & $\mathrm{H}-7$ & LUMO & 0.697 \\
\hline \multirow[t]{2}{*}{$\mathrm{S}_{9}$} & 3.479 & 356 & 0.025 & $\mathrm{H}-11$ & LUMO & -0.263 \\
\hline & & & & $\mathrm{H}-8$ & LUMO & 0.647 \\
\hline \multirow[t]{2}{*}{$\mathrm{S}_{10}$} & 3.485 & 356 & 0.000 & $\mathrm{H}-10$ & LUMO & -0.284 \\
\hline & & & & H-9 & LUMO & 0.634 \\
\hline \multirow[t]{2}{*}{$\mathrm{S}_{11}$} & 3.603 & 344 & 0.011 & H-11 & LUMO & 0.638 \\
\hline & & & & H-8 & LUMO & 0.268 \\
\hline \multirow[t]{2}{*}{$\mathrm{S}_{12}$} & 3.605 & 344 & 0.000 & H-10 & LUMO & 0.631 \\
\hline & & & & H-9 & LUMO & 0.289 \\
\hline $\mathrm{S}_{13}$ & 3.852 & 322 & 0.000 & H-12 & LUMO & 0.700 \\
\hline $\mathrm{S}_{14}$ & 3.853 & 322 & 0.000 & H-13 & LUMO & 0.700 \\
\hline \multirow[t]{2}{*}{$\mathrm{S}_{15}$} & 3.874 & 320 & 0.000 & H-1 & $\mathrm{L}+8$ & -0.128 \\
\hline & & & & HOMO & $\mathrm{L}+1$ & 0.684 \\
\hline
\end{tabular}

Triplet excited states

\begin{tabular}{lllllll}
$\mathrm{T}_{1}$ & 2.771 & 447 & 0.000 & HOMO & LUMO & 0.698 \\
$\mathrm{~T}_{2}$ & 2.782 & 446 & 0.000 & $\mathrm{H}-1$ & LUMO & 0.703 \\
$\mathrm{~T}_{3}$ & 3.119 & 398 & 0.000 & $\mathrm{H}-11$ & LUMO & -0.115 \\
& & & & $\mathrm{H}-2$ & LUMO & 0.676 \\
$\mathrm{~T}_{4}$ & 3.128 & \multirow{2}{*}{396} & \multirow{2}{*}{0.000} & $\mathrm{H}-10$ & LUMO & -0.145 \\
& & & & $\mathrm{H}-3$ & LUMO & 0.684 \\
$\mathrm{~T}_{5}$ & 3.213 & \multirow{2}{*}{386} & \multirow{2}{*}{0.000} & $\mathrm{H}-28$ & LUMO & -0.161 \\
& & & & $\mathrm{H}-8$ & LUMO & 0.109 \\
& & & & H-6 & LUMO & -0.122
\end{tabular}




\section{(Continued)}

\begin{tabular}{|c|c|c|c|c|c|c|}
\hline & & & & $\mathrm{H}-4$ & LUMO & 0.648 \\
\hline $\mathrm{T}_{6}$ & 3.230 & 384 & 0.000 & H-5 & LUMO & 0.704 \\
\hline \multirow[t]{6}{*}{$\mathrm{T}_{7}$} & 3.289 & 377 & 0.000 & H-29 & $\mathrm{L}+1$ & 0.100 \\
\hline & & & & H-28 & LUMO & -0.274 \\
\hline & & & & H-11 & LUMO & -0.314 \\
\hline & & & & H-8 & LUMO & 0.323 \\
\hline & & & & H-6 & LUMO & -0.321 \\
\hline & & & & H-4 & LUMO & -0.257 \\
\hline \multirow[t]{3}{*}{$\mathrm{T}_{8}$} & 3.350 & 370 & 0.000 & H-10 & LUMO & 0.518 \\
\hline & & & & H-9 & LUMO & -0.383 \\
\hline & & & & H-3 & LUMO & 0.161 \\
\hline \multirow[t]{6}{*}{$\mathrm{T}_{9}$} & 3.378 & 367 & 0.000 & H-28 & LUMO & 0.127 \\
\hline & & & & H-24 & LUMO & -0.106 \\
\hline & & & & H-11 & LUMO & -0.395 \\
\hline & & & & H-8 & LUMO & 0.225 \\
\hline & & & & H-6 & LUMO & 0.436 \\
\hline & & & & H-2 & LUMO & -0.164 \\
\hline $\mathrm{T}_{10}$ & 3.415 & 363 & 0.000 & H-7 & LUMO & 0.696 \\
\hline \multirow[t]{4}{*}{$\mathrm{T}_{11}$} & 3.483 & 356 & 0.000 & H-28 & LUMO & -0.213 \\
\hline & & & & H-11 & LUMO & 0.350 \\
\hline & & & & H-8 & LUMO & 0.390 \\
\hline & & & & H-6 & LUMO & 0.334 \\
\hline \multirow[t]{2}{*}{$\mathrm{T}_{12}$} & 3.540 & 350 & 0.000 & H-10 & LUMO & 0.379 \\
\hline & & & & H-9 & LUMO & 0.577 \\
\hline \multirow[t]{7}{*}{$\mathrm{T}_{13}$} & 3.622 & 342 & 0.000 & H-32 & LUMO & -0.158 \\
\hline & & & & H-30 & $\mathrm{L}+8$ & -0.120 \\
\hline & & & & H-29 & $\mathrm{L}+1$ & -0.154 \\
\hline & & & & H-28 & LUMO & 0.365 \\
\hline & & & & H-11 & LUMO & 0.180 \\
\hline & & & & H-8 & LUMO & 0.413 \\
\hline & & & & H-6 & LUMO & -0.264 \\
\hline \multirow[t]{4}{*}{$\mathrm{T}_{14}$} & 3.634 & 341 & 0.000 & H-23 & $\mathrm{L}+17$ & 0.106 \\
\hline & & & & H-21 & $\mathrm{L}+2$ & 0.181 \\
\hline & & & & H-20 & $\mathrm{L}+3$ & -0.179 \\
\hline & & & & H-15 & $\mathrm{L}+2$ & 0.116 \\
\hline
\end{tabular}




\begin{tabular}{|c|c|c|c|c|c|c|}
\hline & & & & $\mathrm{H}-14$ & $\mathrm{~L}+3$ & 0.113 \\
\hline & & & & $\mathrm{H}-7$ & $\mathrm{~L}+2$ & -0.133 \\
\hline & & & & H-6 & $L+3$ & -0.132 \\
\hline & & & & H-5 & $\mathrm{L}+2$ & 0.101 \\
\hline & & & & H-3 & $\mathrm{L}+2$ & 0.117 \\
\hline & & & & $\mathrm{H}-2$ & $\mathrm{~L}+3$ & -0.118 \\
\hline & & & & H-1 & $\mathrm{L}+2$ & 0.237 \\
\hline & & & & HOMO & $\mathrm{L}+3$ & -0.233 \\
\hline \multirow[t]{12}{*}{$\mathrm{T}_{15}$} & 3.634 & 341 & 0.000 & $\mathrm{H}-23$ & $\mathrm{~L}+16$ & -0.105 \\
\hline & & & & H-21 & $\mathrm{L}+3$ & -0.175 \\
\hline & & & & $\mathrm{H}-20$ & $\mathrm{~L}+2$ & 0.181 \\
\hline & & & & H-15 & $\mathrm{L}+3$ & -0.114 \\
\hline & & & & $\mathrm{H}-14$ & $\mathrm{~L}+2$ & -0.114 \\
\hline & & & & H-7 & $\mathrm{L}+3$ & 0.130 \\
\hline & & & & H-6 & $\mathrm{L}+2$ & 0.133 \\
\hline & & & & H-4 & $\mathrm{L}+2$ & -0.100 \\
\hline & & & & H-3 & $\mathrm{L}+3$ & -0.114 \\
\hline & & & & $\mathrm{H}-2$ & $\mathrm{~L}+2$ & 0.119 \\
\hline & & & & H-1 & $L+3$ & -0.226 \\
\hline & & & & HOMO & $\mathrm{L}+2$ & 0.239 \\
\hline
\end{tabular}


Table S19. Energy and components of molecular orbitals around Fermi energy for the model complex $\left[\left\{\left(\mathrm{NH}_{3}\right)\left(\mathrm{PH}_{3}\right)\left(\mathrm{Ag}_{2} \mathrm{Br}_{2}\right)\left(\mathrm{PPh}_{3}\right)\right\}\right.$-bpy- $\left.\left\{\left(\mathrm{PPh}_{3}\right)\left(\mathrm{Ag}_{2} \mathrm{Br}_{2}\right)\left(\mathrm{NH}_{3}\right)\left(\mathrm{PH}_{3}\right)\right\}\right](3-\mathrm{Br})$ in DMSO.

\begin{tabular}{|c|c|c|c|c|c|c|c|c|}
\hline & $\begin{array}{c}\text { Energy } \\
(\mathrm{eV})\end{array}$ & $\mathrm{Br}$ & $\mathrm{Ag}$ & bpy & $\mathrm{P}(\mathrm{Ph})$ & $\mathrm{Ph}$ & $\mathrm{PH}_{3}$ & $\mathrm{NH}_{3}$ \\
\hline $\mathrm{L}+10$ & -0.39 & 0.00 & 0.20 & 0.02 & 0.00 & 0.74 & 0.00 & 0.03 \\
\hline $\mathrm{L}+9$ & -0.39 & 0.01 & 0.20 & 0.00 & 0.00 & 0.76 & 0.00 & 0.03 \\
\hline $\mathrm{L}+8$ & -0.49 & 0.00 & 0.00 & 0.92 & 0.00 & 0.07 & 0.00 & 0.00 \\
\hline $\mathrm{L}+7$ & -0.68 & 0.00 & 0.03 & 0.00 & 0.07 & 0.89 & 0.00 & 0.00 \\
\hline $\mathrm{L}+6$ & -0.68 & 0.00 & 0.03 & 0.00 & 0.07 & 0.89 & 0.00 & 0.00 \\
\hline $\mathrm{L}+5$ & -0.70 & 0.00 & 0.02 & 0.00 & 0.04 & 0.92 & 0.00 & 0.00 \\
\hline $\mathrm{L}+4$ & -0.70 & 0.00 & 0.02 & 0.00 & 0.05 & 0.92 & 0.00 & 0.00 \\
\hline $\mathrm{L}+3$ & -0.81 & 0.00 & 0.03 & 0.02 & 0.05 & 0.90 & 0.00 & 0.00 \\
\hline $\mathrm{L}+2$ & -0.82 & 0.00 & 0.03 & 0.02 & 0.05 & 0.91 & 0.00 & 0.00 \\
\hline $\mathrm{L}+1$ & -1.09 & 0.00 & 0.00 & 0.97 & 0.00 & 0.03 & 0.00 & 0.00 \\
\hline LUMO & -2.23 & 0.00 & 0.00 & 0.99 & 0.00 & 0.00 & 0.00 & 0.00 \\
\hline HOMO & -5.62 & 0.45 & 0.28 & 0.00 & 0.12 & 0.09 & 0.04 & 0.02 \\
\hline H-1 & -5.63 & 0.46 & 0.28 & 0.00 & 0.11 & 0.09 & 0.04 & 0.02 \\
\hline $\mathrm{H}-2$ & -5.89 & 0.36 & 0.36 & 0.00 & 0.02 & 0.03 & 0.03 & 0.20 \\
\hline $\mathrm{H}-3$ & -5.89 & 0.36 & 0.36 & 0.00 & 0.02 & 0.03 & 0.03 & 0.19 \\
\hline $\mathrm{H}-4$ & -6.13 & 0.58 & 0.30 & 0.00 & 0.04 & 0.06 & 0.00 & 0.00 \\
\hline H-5 & -6.13 & 0.59 & 0.30 & 0.00 & 0.04 & 0.05 & 0.00 & 0.00 \\
\hline H-6 & -6.21 & 0.24 & 0.35 & 0.03 & 0.07 & 0.11 & 0.18 & 0.02 \\
\hline H-7 & -6.22 & 0.22 & 0.35 & 0.03 & 0.07 & 0.13 & 0.17 & 0.02 \\
\hline H-8 & -6.40 & 0.43 & 0.33 & 0.20 & 0.00 & 0.00 & 0.02 & 0.02 \\
\hline H-9 & -6.40 & 0.43 & 0.33 & 0.19 & 0.00 & 0.00 & 0.02 & 0.02 \\
\hline H-10 & -6.50 & 0.78 & 0.13 & 0.01 & 0.00 & 0.04 & 0.02 & 0.00 \\
\hline H-11 & -6.50 & 0.78 & 0.13 & 0.01 & 0.00 & 0.04 & 0.02 & 0.00 \\
\hline H-12 & -6.80 & 0.71 & 0.20 & 0.00 & 0.00 & 0.08 & 0.00 & 0.00 \\
\hline $\mathrm{H}-13$ & -6.80 & 0.71 & 0.20 & 0.00 & 0.00 & 0.08 & 0.00 & 0.00 \\
\hline H-14 & -7.11 & 0.20 & 0.08 & 0.01 & 0.00 & 0.65 & 0.04 & 0.01 \\
\hline H-15 & -7.11 & 0.18 & 0.08 & 0.00 & 0.00 & 0.69 & 0.04 & 0.01 \\
\hline H-16 & -7.14 & 0.00 & 0.00 & 0.00 & 0.00 & 0.98 & 0.00 & 0.00 \\
\hline H-17 & -7.14 & 0.00 & 0.00 & 0.00 & 0.00 & 0.98 & 0.00 & 0.00 \\
\hline H-18 & -7.15 & 0.23 & 0.10 & 0.01 & 0.00 & 0.59 & 0.04 & 0.02 \\
\hline H-19 & -7.15 & 0.25 & 0.11 & 0.01 & 0.00 & 0.56 & 0.05 & 0.02 \\
\hline $\mathrm{H}-20$ & -7.30 & 0.04 & 0.06 & 0.03 & 0.00 & 0.86 & 0.00 & 0.00 \\
\hline
\end{tabular}




\section{(Continued)}

\begin{tabular}{lllllllll} 
H-21 & -7.30 & 0.04 & 0.05 & 0.02 & 0.00 & 0.88 & 0.00 & 0.00 \\
H-22 & -7.40 & 0.02 & 0.02 & 0.01 & 0.01 & 0.93 & 0.00 & 0.00 \\
H-23 & -7.40 & 0.02 & 0.01 & 0.00 & 0.01 & 0.94 & 0.00 & 0.00 \\
H-24 & -7.45 & 0.05 & 0.10 & 0.06 & 0.01 & 0.76 & 0.00 & 0.00 \\
H-25 & -7.45 & 0.02 & 0.01 & 0.00 & 0.00 & 0.96 & 0.00 & 0.00 \\
H-26 & -7.45 & 0.04 & 0.18 & 0.06 & 0.00 & 0.69 & 0.00 & 0.00 \\
H-27 & -7.46 & 0.09 & 0.33 & 0.10 & 0.01 & 0.44 & 0.00 & 0.02 \\
H-28 & -7.59 & 0.02 & 0.20 & 0.73 & 0.00 & 0.02 & 0.02 & 0.00 \\
H-29 & -7.77 & 0.00 & 0.00 & 0.99 & 0.00 & 0.00 & 0.00 & 0.00 \\
H-30 & -7.92 & 0.00 & 0.02 & 0.96 & 0.00 & 0.00 & 0.00 & 0.00 \\
H-31 & -7.96 & 0.11 & 0.56 & 0.02 & 0.06 & 0.19 & 0.05 & 0.01 \\
H-32 & -8.08 & 0.08 & 0.42 & 0.22 & 0.07 & 0.18 & 0.03 & 0.00 \\
H-33 & -8.26 & 0.15 & 0.79 & 0.01 & 0.00 & 0.01 & 0.01 & 0.02 \\
H-34 & -8.26 & 0.15 & 0.79 & 0.00 & 0.00 & 0.01 & 0.01 & 0.02 \\
H-35 & -8.28 & 0.12 & 0.83 & 0.02 & 0.00 & 0.00 & 0.00 & 0.01 \\
H-36 & -8.29 & 0.15 & 0.78 & 0.04 & 0.00 & 0.00 & 0.01 & 0.02 \\
H-37 & -8.54 & 0.08 & 0.87 & 0.01 & 0.00 & 0.03 & 0.00 & 0.00 \\
H-38 & -8.55 & 0.08 & 0.87 & 0.01 & 0.00 & 0.03 & 0.00 & 0.00 \\
H-39 & -8.65 & 0.17 & 0.76 & 0.02 & 0.00 & 0.04 & 0.00 & 0.00 \\
H-40 & -8.65 & 0.18 & 0.76 & 0.01 & 0.00 & 0.03 & 0.00 & 0.00 \\
\hline
\end{tabular}


Table S20. Energy and major components of excited states for the model complex $\left[\left\{\left(\mathrm{NH}_{3}\right)\left(\mathrm{PH}_{3}\right)\left(\mathrm{Ag}_{2} \mathrm{Br}_{2}\right)\left(\mathrm{PPh}_{3}\right)\right\}\right.$-bpy- $\left.\left\{\left(\mathrm{PPh}_{3}\right)\left(\mathrm{Ag}_{2} \mathrm{Br}_{2}\right)\left(\mathrm{NH}_{3}\right)\left(\mathrm{PH}_{3}\right)\right\}\right](\mathbf{3}-\mathrm{Br})$ in DMSO.

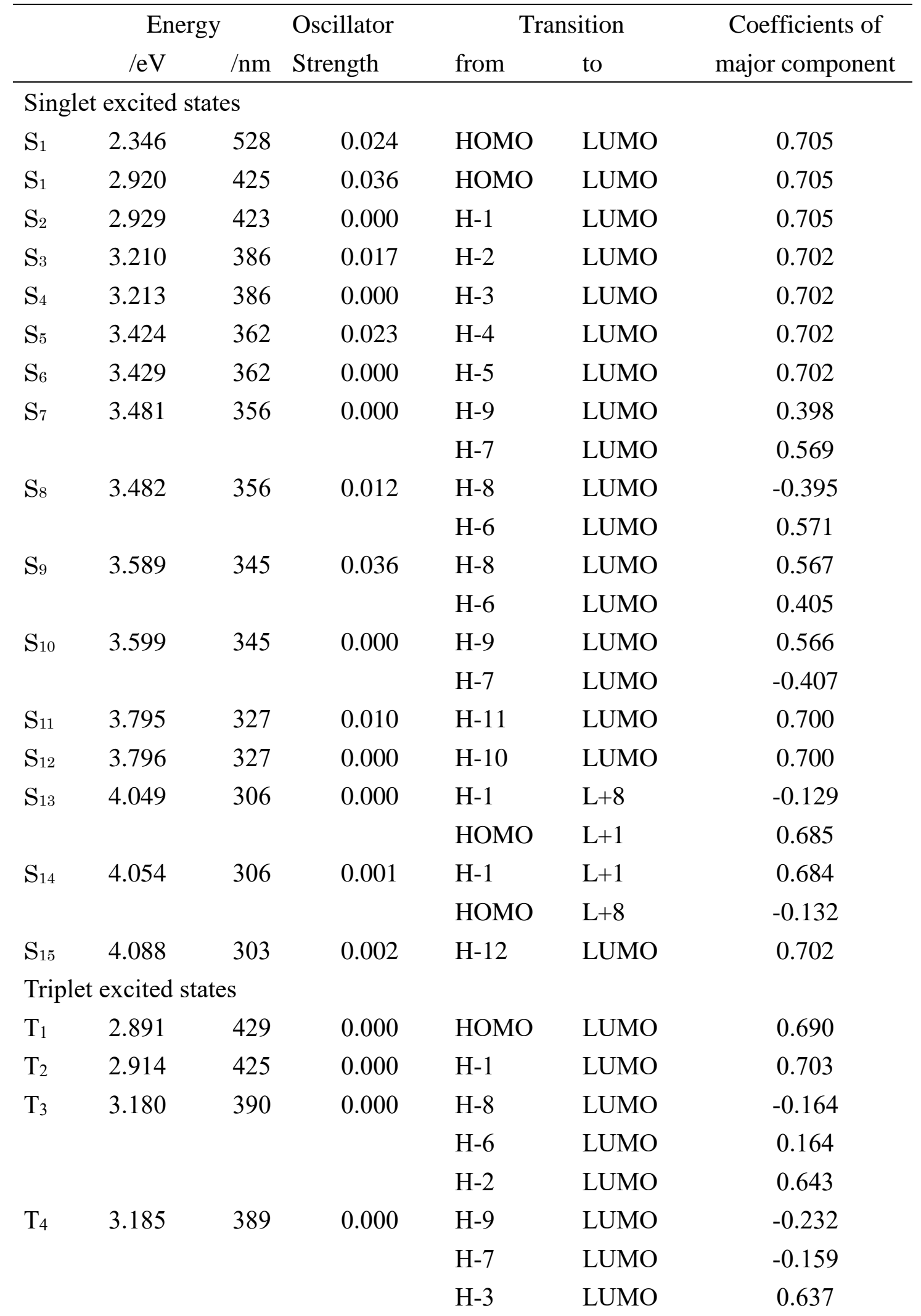


(Continued)

\begin{tabular}{|c|c|c|c|c|c|c|}
\hline \multirow[t]{6}{*}{$\mathrm{T}_{5}$} & 3.281 & 378 & 0.000 & H-28 & LUMO & 0.254 \\
\hline & & & & H-8 & LUMO & 0.553 \\
\hline & & & & H-6 & LUMO & -0.149 \\
\hline & & & & $\mathrm{H}-4$ & LUMO & -0.153 \\
\hline & & & & $\mathrm{H}-2$ & LUMO & 0.134 \\
\hline & & & & HOMO & LUMO & -0.102 \\
\hline \multirow[t]{4}{*}{$\mathrm{T}_{6}$} & 3.306 & 375 & 0.000 & $\mathrm{H}-27$ & LUMO & 0.102 \\
\hline & & & & H-9 & LUMO & 0.506 \\
\hline & & & & $\mathrm{H}-7$ & LUMO & 0.325 \\
\hline & & & & H-3 & LUMO & 0.300 \\
\hline \multirow[t]{7}{*}{$\mathrm{T}_{7}$} & 3.338 & 371 & 0.000 & $\mathrm{H}-32$ & LUMO & 0.105 \\
\hline & & & & H-29 & $\mathrm{L}+1$ & 0.109 \\
\hline & & & & H-28 & LUMO & -0.288 \\
\hline & & & & $\mathrm{H}-8$ & LUMO & 0.125 \\
\hline & & & & H-6 & LUMO & -0.340 \\
\hline & & & & $\mathrm{H}-4$ & LUMO & 0.403 \\
\hline & & & & $\mathrm{H}-2$ & LUMO & 0.234 \\
\hline $\mathrm{T}_{8}$ & 3.418 & 363 & 0.000 & H-5 & LUMO & 0.703 \\
\hline \multirow[t]{4}{*}{$\mathrm{T}_{9}$} & 3.451 & 359 & 0.000 & H-28 & LUMO & 0.207 \\
\hline & & & & H-8 & LUMO & 0.128 \\
\hline & & & & H-6 & LUMO & 0.348 \\
\hline & & & & H-4 & LUMO & 0.536 \\
\hline \multirow[t]{2}{*}{$\mathrm{T}_{10}$} & 3.555 & 349 & 0.000 & H-9 & LUMO & -0.359 \\
\hline & & & & H-7 & LUMO & 0.596 \\
\hline \multirow[t]{7}{*}{$\mathrm{T}_{11}$} & 3.649 & 340 & 0.000 & H-32 & LUMO & 0.137 \\
\hline & & & & H-29 & $\mathrm{L}+1$ & 0.129 \\
\hline & & & & H-28 & LUMO & -0.328 \\
\hline & & & & H-11 & LUMO & 0.193 \\
\hline & & & & H-8 & LUMO & 0.247 \\
\hline & & & & H-6 & LUMO & 0.447 \\
\hline & & & & $\mathrm{H}-4$ & LUMO & -0.141 \\
\hline \multirow[t]{4}{*}{$\mathrm{T}_{12}$} & 3.778 & 328 & 0.000 & $\mathrm{H}-17$ & $\mathrm{~L}+2$ & 0.116 \\
\hline & & & & H-16 & $\mathrm{L}+3$ & 0.118 \\
\hline & & & & $\mathrm{H}-10$ & LUMO & -0.111 \\
\hline & & & & $\mathrm{H}-7$ & $\mathrm{~L}+2$ & -0.118 \\
\hline
\end{tabular}




\begin{tabular}{|c|c|c|c|c|c|c|}
\hline & & & & H-6 & $\mathrm{L}+3$ & 0.109 \\
\hline & & & & H-1 & $\mathrm{L}+2$ & 0.199 \\
\hline & & & & HOMO & $\mathrm{L}+3$ & 0.196 \\
\hline \multirow[t]{8}{*}{$\mathrm{T}_{13}$} & 3.778 & 328 & 0.000 & H-23 & $\mathrm{L}+7$ & 0.103 \\
\hline & & & & $\mathrm{H}-17$ & $\mathrm{~L}+3$ & 0.114 \\
\hline & & & & H-16 & $\mathrm{L}+2$ & 0.123 \\
\hline & & & & H-16 & $\mathrm{L}+14$ & 0.102 \\
\hline & & & & H-7 & $\mathrm{L}+3$ & -0.117 \\
\hline & & & & H-6 & $\mathrm{L}+2$ & 0.113 \\
\hline & & & & $\mathrm{H}-4$ & $\mathrm{~L}+2$ & -0.103 \\
\hline & & & & H-1 & $\mathrm{L}+3$ & 0.195 \\
\hline $\mathrm{T}_{14}$ & 3.780 & 328 & 0.000 & H-10 & LUMO & 0.681 \\
\hline \multirow[t]{3}{*}{$\mathrm{T}_{15}$} & 3.792 & 327 & 0.000 & H-28 & LUMO & 0.114 \\
\hline & & & & H-11 & LUMO & 0.668 \\
\hline & & & & H-8 & LUMO & -0.133 \\
\hline
\end{tabular}


Table S21. Energy and components of molecular orbitals around Fermi energy for the model complex $\left[\left\{\left(\mathrm{NH}_{3}\right)\left(\mathrm{PH}_{3}\right)\left(\mathrm{Ag}_{2} \mathrm{Cl}_{2}\right)\left(\mathrm{PPh}_{3}\right)\right\}\right.$-bpy- $\left.\left\{\left(\mathrm{PPh}_{3}\right)\left(\mathrm{Ag}_{2} \mathrm{Cl}_{2}\right)\left(\mathrm{NH}_{3}\right)\left(\mathrm{PH}_{3}\right)\right\}\right](\mathbf{3}-\mathrm{Cl})$ in DMSO.

\begin{tabular}{|c|c|c|c|c|c|c|c|c|}
\hline & $\begin{array}{c}\text { Energy } \\
(\mathrm{eV})\end{array}$ & $\mathrm{Cl}$ & $\mathrm{Ag}$ & bpy & $\mathrm{P}(\mathrm{Ph})$ & $\mathrm{Ph}$ & $\mathrm{PH}_{3}$ & $\mathrm{NH}_{3}$ \\
\hline $\mathrm{L}+10$ & -0.44 & 0.00 & 0.15 & 0.00 & 0.00 & 0.82 & 0.00 & 0.01 \\
\hline $\mathrm{L}+9$ & -0.45 & 0.00 & 0.14 & 0.04 & 0.00 & 0.80 & 0.00 & 0.01 \\
\hline $\mathrm{L}+8$ & -0.61 & 0.00 & 0.01 & 0.84 & 0.00 & 0.14 & 0.00 & 0.00 \\
\hline $\mathrm{L}+7$ & -0.71 & 0.00 & 0.03 & 0.00 & 0.04 & 0.92 & 0.00 & 0.00 \\
\hline $\mathrm{L}+6$ & -0.74 & 0.00 & 0.03 & 0.07 & 0.05 & 0.85 & 0.00 & 0.00 \\
\hline $\mathrm{L}+5$ & -0.79 & 0.00 & 0.03 & 0.01 & 0.05 & 0.91 & 0.00 & 0.00 \\
\hline $\mathrm{L}+4$ & -0.81 & 0.00 & 0.03 & 0.04 & 0.05 & 0.88 & 0.00 & 0.00 \\
\hline $\mathrm{L}+3$ & -0.82 & 0.00 & 0.01 & 0.02 & 0.07 & 0.90 & 0.00 & 0.00 \\
\hline $\mathrm{L}+2$ & -0.83 & 0.00 & 0.00 & 0.03 & 0.07 & 0.89 & 0.00 & 0.00 \\
\hline $\mathrm{L}+1$ & -1.06 & 0.00 & 0.00 & 0.94 & 0.00 & 0.05 & 0.00 & 0.00 \\
\hline LUMO & -2.19 & 0.00 & 0.00 & 0.99 & 0.00 & 0.00 & 0.00 & 0.00 \\
\hline HOMO & -5.76 & 0.23 & 0.41 & 0.01 & 0.03 & 0.03 & 0.12 & 0.16 \\
\hline H-1 & -5.78 & 0.32 & 0.35 & 0.00 & 0.12 & 0.12 & 0.02 & 0.07 \\
\hline H-2 & -5.85 & 0.23 & 0.37 & 0.00 & 0.16 & 0.15 & 0.00 & 0.08 \\
\hline H-3 & -5.92 & 0.23 & 0.40 & 0.00 & 0.05 & 0.06 & 0.07 & 0.19 \\
\hline H-4 & -6.21 & 0.58 & 0.34 & 0.00 & 0.02 & 0.03 & 0.00 & 0.01 \\
\hline H-5 & -6.22 & 0.60 & 0.35 & 0.00 & 0.01 & 0.03 & 0.00 & 0.00 \\
\hline H-6 & -6.28 & 0.23 & 0.41 & 0.12 & 0.03 & 0.07 & 0.14 & 0.00 \\
\hline H-7 & -6.32 & 0.12 & 0.40 & 0.10 & 0.07 & 0.15 & 0.15 & 0.01 \\
\hline H-8 & -6.51 & 0.34 & 0.38 & 0.16 & 0.00 & 0.03 & 0.07 & 0.00 \\
\hline $\mathrm{H}-9$ & -6.65 & 0.48 & 0.32 & 0.08 & 0.01 & 0.06 & 0.03 & 0.01 \\
\hline H-10 & -6.73 & 0.51 & 0.39 & 0.05 & 0.00 & 0.03 & 0.00 & 0.01 \\
\hline $\mathrm{H}-11$ & -6.75 & 0.79 & 0.18 & 0.00 & 0.00 & 0.00 & 0.00 & 0.00 \\
\hline H-12 & -6.82 & 0.65 & 0.22 & 0.02 & 0.00 & 0.07 & 0.02 & 0.00 \\
\hline H-13 & -6.84 & 0.62 & 0.32 & 0.00 & 0.00 & 0.04 & 0.00 & 0.00 \\
\hline H-14 & -7.12 & 0.00 & 0.00 & 0.00 & 0.00 & 0.99 & 0.00 & 0.00 \\
\hline H-15 & -7.14 & 0.02 & 0.02 & 0.00 & 0.00 & 0.95 & 0.00 & 0.00 \\
\hline H-16 & -7.17 & 0.01 & 0.00 & 0.00 & 0.00 & 0.97 & 0.00 & 0.00 \\
\hline H-17 & -7.17 & 0.04 & 0.03 & 0.00 & 0.00 & 0.92 & 0.00 & 0.00 \\
\hline H-18 & -7.23 & 0.41 & 0.17 & 0.02 & 0.00 & 0.33 & 0.05 & 0.02 \\
\hline H-19 & -7.27 & 0.27 & 0.14 & 0.00 & 0.00 & 0.54 & 0.04 & 0.00 \\
\hline $\mathrm{H}-20$ & -7.36 & 0.01 & 0.02 & 0.01 & 0.00 & 0.95 & 0.00 & 0.00 \\
\hline
\end{tabular}


(Continued)

\begin{tabular}{lllllllll} 
H-21 & -7.36 & 0.01 & 0.03 & 0.00 & 0.00 & 0.95 & 0.00 & 0.00 \\
H-22 & -7.38 & 0.03 & 0.07 & 0.02 & 0.00 & 0.87 & 0.00 & 0.00 \\
H-23 & -7.39 & 0.04 & 0.03 & 0.00 & 0.00 & 0.92 & 0.00 & 0.00 \\
H-24 & -7.41 & 0.09 & 0.04 & 0.02 & 0.00 & 0.84 & 0.00 & 0.00 \\
H-25 & -7.47 & 0.07 & 0.02 & 0.00 & 0.00 & 0.89 & 0.00 & 0.00 \\
H-26 & -7.53 & 0.08 & 0.28 & 0.10 & 0.01 & 0.50 & 0.00 & 0.01 \\
H-27 & -7.58 & 0.17 & 0.28 & 0.28 & 0.01 & 0.24 & 0.00 & 0.00 \\
H-28 & -7.60 & 0.06 & 0.22 & 0.59 & 0.00 & 0.11 & 0.00 & 0.00 \\
H-29 & -7.75 & 0.00 & 0.00 & 0.98 & 0.00 & 0.00 & 0.00 & 0.00 \\
H-30 & -7.86 & 0.00 & 0.03 & 0.96 & 0.00 & 0.00 & 0.00 & 0.00 \\
H-31 & -7.89 & 0.19 & 0.68 & 0.05 & 0.01 & 0.04 & 0.00 & 0.02 \\
H-32 & -8.02 & 0.15 & 0.66 & 0.03 & 0.04 & 0.11 & 0.00 & 0.00 \\
H-33 & -8.06 & 0.09 & 0.83 & 0.00 & 0.01 & 0.04 & 0.02 & 0.01 \\
H-34 & -8.07 & 0.16 & 0.73 & 0.02 & 0.01 & 0.04 & 0.02 & 0.02 \\
H-35 & -8.12 & 0.09 & 0.82 & 0.03 & 0.00 & 0.02 & 0.01 & 0.01 \\
H-36 & -8.18 & 0.13 & 0.73 & 0.05 & 0.02 & 0.05 & 0.01 & 0.01 \\
H-37 & -8.40 & 0.14 & 0.78 & 0.04 & 0.00 & 0.03 & 0.00 & 0.00 \\
H-38 & -8.47 & 0.12 & 0.78 & 0.06 & 0.00 & 0.03 & 0.00 & 0.00 \\
H-39 & -8.69 & 0.32 & 0.51 & 0.12 & 0.00 & 0.00 & 0.02 & 0.00 \\
H-40 & -8.72 & 0.26 & 0.70 & 0.00 & 0.00 & 0.02 & 0.00 & 0.00 \\
\hline & & & & & & & &
\end{tabular}


Table S22. Energy and major components of excited states for the model complex $\left[\left\{\left(\mathrm{NH}_{3}\right)\left(\mathrm{PH}_{3}\right)\left(\mathrm{Ag}_{2} \mathrm{Cl}_{2}\right)\left(\mathrm{PPh}_{3}\right)\right\}\right.$-bpy- $\left.\left\{\left(\mathrm{PPh}_{3}\right)\left(\mathrm{Ag}_{2} \mathrm{Cl}_{2}\right)\left(\mathrm{NH}_{3}\right)\left(\mathrm{PH}_{3}\right)\right\}\right](\mathbf{3}-\mathbf{C l})$ in DMSO.

\begin{tabular}{|c|c|c|c|c|c|c|}
\hline & \multicolumn{2}{|c|}{ Energy } & \multirow{2}{*}{$\begin{array}{l}\text { Oscillator } \\
\text { Strength }\end{array}$} & \multicolumn{2}{|c|}{ Transition } & \multirow{2}{*}{$\begin{array}{l}\text { Coefficients of } \\
\text { major component }\end{array}$} \\
\hline & leV & $/ \mathrm{nm}$ & & from & to & \\
\hline \multicolumn{7}{|c|}{ Singlet excited states } \\
\hline \multirow[t]{2}{*}{$\mathrm{S}_{1}$} & 3.121 & 397 & 0.024 & $\mathrm{H}-1$ & LUMO & 0.667 \\
\hline & & & & HOMO & LUMO & 0.203 \\
\hline \multirow[t]{3}{*}{$\mathrm{S}_{2}$} & 3.133 & 396 & 0.004 & $\mathrm{H}-2$ & LUMO & 0.282 \\
\hline & & & & $\mathrm{H}-1$ & LUMO & -0.214 \\
\hline & & & & HOMO & LUMO & 0.604 \\
\hline \multirow[t]{2}{*}{$\mathrm{S}_{3}$} & 3.221 & 385 & 0.005 & $\mathrm{H}-2$ & LUMO & 0.641 \\
\hline & & & & HOMO & LUMO & -0.293 \\
\hline $\mathrm{S}_{4}$ & 3.295 & 376 & 0.011 & $\mathrm{H}-3$ & LUMO & 0.699 \\
\hline \multirow[t]{3}{*}{$\mathrm{S}_{5}$} & 3.537 & 351 & 0.001 & $\mathrm{H}-9$ & LUMO & 0.137 \\
\hline & & & & H-6 & LUMO & 0.598 \\
\hline & & & & $\mathrm{H}-4$ & LUMO & -0.302 \\
\hline \multirow[t]{3}{*}{$\mathrm{S}_{6}$} & 3.547 & 350 & 0.003 & $\mathrm{H}-8$ & LUMO & 0.140 \\
\hline & & & & $\mathrm{H}-7$ & LUMO & 0.249 \\
\hline & & & & $\mathrm{H}-5$ & LUMO & 0.633 \\
\hline \multirow[t]{5}{*}{$\mathrm{S}_{7}$} & 3.562 & 348 & 0.010 & $\mathrm{H}-8$ & LUMO & 0.173 \\
\hline & & & & $\mathrm{H}-7$ & LUMO & 0.289 \\
\hline & & & & H-6 & LUMO & 0.239 \\
\hline & & & & H-5 & LUMO & -0.204 \\
\hline & & & & $\mathrm{H}-4$ & LUMO & 0.525 \\
\hline \multirow[t]{5}{*}{$\mathrm{S}_{8}$} & 3.565 & 348 & 0.004 & H-8 & LUMO & 0.264 \\
\hline & & & & H-7 & LUMO & 0.461 \\
\hline & & & & H-6 & LUMO & -0.182 \\
\hline & & & & $\mathrm{H}-5$ & LUMO & -0.220 \\
\hline & & & & $\mathrm{H}-4$ & LUMO & -0.347 \\
\hline \multirow[t]{2}{*}{$\mathrm{S}_{9}$} & 3.803 & 326 & 0.015 & H-8 & LUMO & 0.604 \\
\hline & & & & H-7 & LUMO & -0.359 \\
\hline \multirow[t]{3}{*}{$S_{10}$} & 3.959 & 313 & 0.005 & H-10 & LUMO & 0.232 \\
\hline & & & & H-9 & LUMO & 0.635 \\
\hline & & & & H-6 & LUMO & -0.181 \\
\hline \multirow[t]{2}{*}{$\mathrm{S}_{11}$} & 4.061 & 305 & 0.025 & H-11 & LUMO & -0.160 \\
\hline & & & & H-10 & LUMO & 0.634 \\
\hline
\end{tabular}




\section{(Continued)}

$\begin{array}{lllllll} & & & & \text { H-9 } & \text { LUMO } & -0.241 \\ \mathrm{~S}_{12} & 4.069 & 305 & 0.001 & \text { H-11 } & \text { LUMO } & 0.685 \\ & & & & \text { H-10 } & \text { LUMO } & 0.143 \\ \mathrm{~S}_{13} & 4.157 & 298 & 0.001 & \text { H-12 } & \text { LUMO } & 0.695 \\ \mathrm{~S}_{14} & 4.166 & 298 & 0.004 & \text { H-13 } & \text { LUMO } & 0.702 \\ \mathrm{~S}_{15} & 4.219 & 294 & 0.002 & \text { H-6 } & \text { L+1 } & -0.102 \\ & & & & \text { H-2 } & \text { L+1 } & 0.325 \\ & & & & \text { HOMO } & \text { L+1 } & 0.569 \\ & & & & \text { HOMO } & \text { L+8 } & -0.115\end{array}$

Triplet excited states

\begin{tabular}{|c|c|c|c|c|c|c|}
\hline \multirow[t]{5}{*}{$\mathrm{T}_{1}$} & 3.067 & 404 & 0.000 & $\mathrm{H}-27$ & LUMO & -0.101 \\
\hline & & & & H-6 & LUMO & -0.190 \\
\hline & & & & H-2 & LUMO & 0.307 \\
\hline & & & & $\mathrm{H}-1$ & LUMO & 0.247 \\
\hline & & & & HOMO & LUMO & 0.509 \\
\hline \multirow[t]{4}{*}{$\mathrm{T}_{2}$} & 3.099 & 400 & 0.000 & H-3 & LUMO & 0.103 \\
\hline & & & & H-2 & LUMO & -0.131 \\
\hline & & & & H-1 & LUMO & 0.629 \\
\hline & & & & HOMO & LUMO & -0.216 \\
\hline \multirow[t]{2}{*}{$\mathrm{T}_{3}$} & 3.212 & 386 & 0.000 & $\mathrm{H}-2$ & LUMO & 0.578 \\
\hline & & & & HOMO & LUMO & -0.374 \\
\hline \multirow[t]{5}{*}{$\mathrm{T}_{4}$} & 3.247 & 382 & 0.000 & H-8 & LUMO & -0.187 \\
\hline & & & & H-7 & LUMO & -0.274 \\
\hline & & & & $\mathrm{H}-3$ & LUMO & 0.561 \\
\hline & & & & $\mathrm{H}-2$ & LUMO & -0.138 \\
\hline & & & & H-1 & LUMO & -0.116 \\
\hline \multirow[t]{6}{*}{$\mathrm{T}_{5}$} & 3.352 & 370 & 0.000 & H-28 & LUMO & 0.147 \\
\hline & & & & H-8 & LUMO & 0.390 \\
\hline & & & & $\mathrm{H}-7$ & LUMO & 0.385 \\
\hline & & & & H-6 & LUMO & 0.142 \\
\hline & & & & H-3 & LUMO & 0.273 \\
\hline & & & & H-1 & LUMO & -0.131 \\
\hline \multirow[t]{3}{*}{$\mathrm{T}_{6}$} & 3.378 & 367 & 0.000 & $\mathrm{H}-10$ & LUMO & 0.133 \\
\hline & & & & H-9 & LUMO & 0.206 \\
\hline & & & & $\mathrm{H}-7$ & LUMO & -0.142 \\
\hline
\end{tabular}


(Continued)

\begin{tabular}{|c|c|c|c|c|c|c|}
\hline & & & & H-6 & LUMO & 0.525 \\
\hline & & & & $\mathrm{H}-4$ & LUMO & -0.135 \\
\hline & & & & H-3 & LUMO & -0.130 \\
\hline & & & & $\mathrm{H}-2$ & LUMO & 0.117 \\
\hline & & & & HOMO & LUMO & 0.204 \\
\hline \multirow[t]{11}{*}{$\mathrm{T}_{7}$} & 3.421 & 362 & 0.000 & $\mathrm{H}-30$ & $\mathrm{~L}+8$ & -0.136 \\
\hline & & & & H-29 & $\mathrm{L}+1$ & -0.174 \\
\hline & & & & $\mathrm{H}-28$ & LUMO & 0.400 \\
\hline & & & & $\mathrm{H}-27$ & LUMO & -0.242 \\
\hline & & & & H-26 & LUMO & -0.109 \\
\hline & & & & $\mathrm{H}-10$ & LUMO & 0.116 \\
\hline & & & & $\mathrm{H}-7$ & LUMO & -0.144 \\
\hline & & & & H-6 & LUMO & -0.140 \\
\hline & & & & $\mathrm{H}-4$ & LUMO & -0.110 \\
\hline & & & & $\mathrm{H}-3$ & LUMO & -0.257 \\
\hline & & & & $\mathrm{H}-2$ & LUMO & -0.129 \\
\hline $\mathrm{T}_{8}$ & 3.547 & 350 & 0.000 & H-5 & LUMO & 0.702 \\
\hline \multirow[t]{2}{*}{$\mathrm{T}_{9}$} & 3.562 & 348 & 0.000 & H-6 & LUMO & 0.121 \\
\hline & & & & $\mathrm{H}-4$ & LUMO & 0.675 \\
\hline \multirow[t]{12}{*}{$\mathrm{T}_{10}$} & 3.713 & 334 & 0.000 & $\mathrm{H}-22$ & $\mathrm{~L}+2$ & -0.148 \\
\hline & & & & H-22 & $\mathrm{L}+3$ & 0.104 \\
\hline & & & & $\mathrm{H}-22$ & $\mathrm{~L}+7$ & -0.174 \\
\hline & & & & $\mathrm{H}-20$ & $\mathrm{~L}+14$ & 0.134 \\
\hline & & & & H-18 & $\mathrm{L}+2$ & -0.135 \\
\hline & & & & H-16 & $\mathrm{L}+14$ & 0.101 \\
\hline & & & & H-7 & $\mathrm{L}+2$ & -0.175 \\
\hline & & & & $\mathrm{H}-7$ & $\mathrm{~L}+3$ & 0.104 \\
\hline & & & & H-3 & $\mathrm{L}+2$ & 0.161 \\
\hline & & & & H-1 & $\mathrm{L}+2$ & 0.272 \\
\hline & & & & H-1 & $\mathrm{L}+3$ & -0.158 \\
\hline & & & & $\mathrm{H}-1$ & $\mathrm{~L}+7$ & 0.110 \\
\hline \multirow[t]{4}{*}{$\mathrm{T}_{11}$} & 3.738 & 332 & 0.000 & H-25 & $\mathrm{L}+6$ & 0.117 \\
\hline & & & & $\mathrm{H}-23$ & $\mathrm{~L}+16$ & 0.123 \\
\hline & & & & $\mathrm{H}-21$ & $\mathrm{~L}+3$ & -0.112 \\
\hline & & & & $\mathrm{H}-21$ & $\mathrm{~L}+5$ & 0.132 \\
\hline
\end{tabular}


(Continued)

\begin{tabular}{|c|c|c|c|c|c|c|}
\hline & & & & H-21 & $\mathrm{L}+9$ & -0.123 \\
\hline & & & & H-19 & $L+16$ & 0.112 \\
\hline & & & & H-17 & $\mathrm{L}+5$ & -0.125 \\
\hline & & & & $\mathrm{H}-15$ & $\mathrm{~L}+12$ & 0.120 \\
\hline & & & & H-6 & $\mathrm{L}+3$ & -0.120 \\
\hline & & & & $\mathrm{H}-2$ & $\mathrm{~L}+2$ & 0.153 \\
\hline & & & & $\mathrm{H}-2$ & $\mathrm{~L}+3$ & 0.271 \\
\hline & & & & $\mathrm{H}-2$ & $\mathrm{~L}+5$ & -0.135 \\
\hline & & & & $\mathrm{H}-2$ & $\mathrm{~L}+6$ & -0.123 \\
\hline & & & & HOMO & $\mathrm{L}+3$ & 0.134 \\
\hline $\mathrm{T}_{12}$ & 3.745 & 331 & 0.000 & H-24 & $\mathrm{L}+2$ & -0.101 \\
\hline & & & & $\mathrm{H}-24$ & $\mathrm{~L}+4$ & 0.128 \\
\hline & & & & $\mathrm{H}-24$ & $\mathrm{~L}+7$ & -0.102 \\
\hline & & & & H-24 & $\mathrm{L}+10$ & 0.122 \\
\hline & & & & H-22 & $\mathrm{L}+10$ & -0.103 \\
\hline & & & & $\mathrm{H}-20$ & $L+13$ & 0.102 \\
\hline & & & & H-16 & $\mathrm{L}+4$ & 0.111 \\
\hline & & & & H-16 & $\mathrm{L}+13$ & -0.141 \\
\hline & & & & H-16 & $\mathrm{L}+14$ & 0.126 \\
\hline & & & & H-14 & $\mathrm{L}+7$ & -0.125 \\
\hline & & & & H-14 & $\mathrm{L}+10$ & 0.108 \\
\hline & & & & $\mathrm{H}-7$ & $\mathrm{~L}+4$ & -0.137 \\
\hline & & & & $\mathrm{H}-3$ & $\mathrm{~L}+4$ & 0.137 \\
\hline & & & & H-1 & $\mathrm{L}+4$ & 0.236 \\
\hline & & & & H-1 & $\mathrm{L}+5$ & -0.119 \\
\hline & & & & $\mathrm{H}-1$ & $\mathrm{~L}+7$ & -0.113 \\
\hline & & & & H-1 & $L+10$ & 0.103 \\
\hline $\mathrm{T}_{13}$ & 3.765 & 329 & 0.000 & H-25 & $\mathrm{L}+9$ & 0.103 \\
\hline & & & & $\mathrm{H}-23$ & $\mathrm{~L}+12$ & -0.162 \\
\hline & & & & H-21 & $\mathrm{L}+3$ & 0.125 \\
\hline & & & & $\mathrm{H}-17$ & $L+16$ & 0.117 \\
\hline & & & & H-15 & $\mathrm{L}+5$ & 0.132 \\
\hline & & & & H-15 & $\mathrm{L}+6$ & 0.147 \\
\hline & & & & H-15 & $L+16$ & -0.121 \\
\hline & & & & H-6 & $\mathrm{L}+5$ & -0.102 \\
\hline
\end{tabular}




\begin{tabular}{|c|c|c|c|c|c|c|}
\hline \multicolumn{4}{|c|}{ (Continued) } & $\mathrm{H}-2$ & $\mathrm{~L}+4$ & 0.157 \\
\hline & & & & $\mathrm{H}-2$ & $\mathrm{~L}+5$ & 0.225 \\
\hline & & & & $\mathrm{H}-2$ & $\mathrm{~L}+6$ & -0.153 \\
\hline & & & & HOMO & $\mathrm{L}+5$ & 0.107 \\
\hline \multirow[t]{2}{*}{$\mathrm{T}_{14}$} & 3.791 & 327 & 0.000 & $\mathrm{H}-8$ & LUMO & 0.494 \\
\hline & & & & H-7 & LUMO & -0.451 \\
\hline \multirow[t]{13}{*}{$\mathrm{T}_{15}$} & 3.812 & 325 & 0.000 & $\mathrm{H}-25$ & $\mathrm{~L}+5$ & 0.179 \\
\hline & & & & $\mathrm{H}-25$ & $\mathrm{~L}+9$ & 0.102 \\
\hline & & & & $\mathrm{H}-23$ & $\mathrm{~L}+3$ & 0.114 \\
\hline & & & & $\mathrm{H}-23$ & $\mathrm{~L}+6$ & 0.130 \\
\hline & & & & $\mathrm{H}-21$ & $\mathrm{~L}+6$ & 0.141 \\
\hline & & & & $\mathrm{H}-17$ & $\mathrm{~L}+5$ & 0.111 \\
\hline & & & & $\mathrm{H}-17$ & $\mathrm{~L}+12$ & -0.113 \\
\hline & & & & $\mathrm{H}-17$ & $\mathrm{~L}+16$ & -0.114 \\
\hline & & & & $\mathrm{H}-15$ & $\mathrm{~L}+3$ & 0.127 \\
\hline & & & & $\mathrm{H}-15$ & $\mathrm{~L}+9$ & 0.115 \\
\hline & & & & $\mathrm{H}-15$ & $\mathrm{~L}+12$ & -0.180 \\
\hline & & & & $\mathrm{H}-2$ & $\mathrm{~L}+6$ & -0.170 \\
\hline & & & & $\mathrm{H}-2$ & $\mathrm{~L}+9$ & 0.158 \\
\hline
\end{tabular}


Table S23. MOs related to the $\pi$ and $\pi^{*}$ orbitals of bpy and HOMO in complexes $\left[\left\{\left(\mathrm{NH}_{3}\right)\left(\mathrm{PH}_{3}\right)\left(\mathrm{Ag}_{2} \mathrm{X}_{2}\right)\left(\mathrm{PPh}_{3}\right)\right\}\right.$-bpy- $\left.\left\{\left(\mathrm{PPh}_{3}\right)\left(\mathrm{Ag}_{2} \mathrm{X}_{2}\right)\left(\mathrm{NH}_{3}\right)\left(\mathrm{PH}_{3}\right)\right\}\right](\mathbf{3}-\mathbf{X})$ in vacuum.

$\Delta(\mathrm{eV})^{a} \begin{gathered}\begin{array}{c}\text { Energy } \\ (\mathrm{eV})\end{array} \\ \mathrm{N}\end{gathered}$

\begin{tabular}{lccccccccc}
\hline 3-I & & & & & & & & & \\
L+1 & 1.08 & -1.11 & 0.00 & 0.00 & $\mathbf{0 . 9 9}$ & 0.00 & 0.01 & 0.00 & 0.00 \\
LUMO & 0.00 & -2.19 & 0.00 & 0.00 & $\mathbf{0 . 9 9}$ & 0.00 & 0.00 & 0.00 & 0.00 \\
HOMO & -2.67 & -4.86 & $\mathbf{0 . 6 3}$ & $\mathbf{0 . 2 1}$ & $\mathbf{0 . 0 0}$ & 0.07 & 0.04 & 0.03 & 0.01 \\
H-28 & -5.26 & -7.45 & 0.02 & 0.37 & $\mathbf{0 . 4 3}$ & 0.01 & 0.12 & 0.04 & 0.00 \\
H-30 & -5.54 & -7.73 & 0.00 & 0.01 & $\mathbf{0 . 9 8}$ & 0.00 & 0.00 & 0.00 & 0.00 \\
H-31 & -5.64 & -7.83 & 0.15 & 0.35 & $\mathbf{0 . 3 9}$ & 0.01 & 0.03 & 0.04 & 0.02 \\
H-32 & -5.66 & -7.85 & 0.13 & 0.48 & $\mathbf{0 . 2 1}$ & 0.05 & 0.12 & 0.00 & 0.00 \\
H-34 & -5.70 & -7.89 & 0.01 & 0.04 & $\mathbf{0 . 9 4}$ & 0.00 & 0.00 & 0.00 & 0.00
\end{tabular}

\section{3-Br}

$\begin{array}{llllllllll}\text { L+1 } & 1.13 & -1.01 & 0.00 & 0.00 & \mathbf{0 . 9 8} & 0.00 & 0.01 & 0.00 & 0.00 \\ \text { LUMO } & 0.00 & -2.14 & 0.00 & 0.00 & \mathbf{0 . 9 8} & 0.00 & 0.00 & 0.00 & 0.00 \\ \text { HOMO } & -2.81 & -4.95 & \mathbf{0 . 5 7} & \mathbf{0 . 2 5} & \mathbf{0 . 0 0} & 0.08 & 0.05 & 0.03 & 0.01 \\ \text { H-28 } & -5.24 & -7.38 & 0.03 & 0.33 & \mathbf{0 . 4 6} & 0.00 & 0.13 & 0.04 & 0.00 \\ \text { H-30 } & -5.53 & -7.67 & 0.15 & 0.45 & \mathbf{0 . 3 2} & 0.01 & 0.06 & 0.00 & 0.01 \\ \text { H-32 } & -5.56 & -7.70 & 0.07 & 0.19 & \mathbf{0 . 7 0} & 0.00 & 0.02 & 0.00 & 0.00 \\ \text { H-33 } & -5.61 & -7.75 & 0.04 & 0.35 & \mathbf{0 . 4 2} & 0.04 & 0.12 & 0.01 & 0.00 \\ \text { H-35 } & -5.71 & -7.85 & 0.00 & 0.59 & \mathbf{0 . 3 8} & 0.00 & 0.00 & 0.00 & 0.00 \\ \text { H-36 } & -5.74 & -7.88 & 0.01 & 0.31 & \mathbf{0 . 6 2} & 0.01 & 0.03 & 0.00 & 0.00\end{array}$

\section{3-Cl}

\begin{tabular}{llllllllll} 
L+1 & 1.09 & -0.89 & 0.00 & 0.00 & $\mathbf{0 . 9 2}$ & 0.00 & 0.07 & 0.00 & 0.00 \\
LUMO & 0.00 & -1.98 & 0.00 & 0.00 & $\mathbf{0 . 9 9}$ & 0.00 & 0.00 & 0.00 & 0.00 \\
HOMO & -2.98 & -4.96 & $\mathbf{0 . 6 2}$ & $\mathbf{0 . 2 7}$ & $\mathbf{0 . 0 0}$ & 0.03 & 0.02 & 0.03 & 0.02 \\
H-28 & -5.28 & -7.26 & 0.06 & 0.36 & $\mathbf{0 . 4 2}$ & 0.00 & 0.14 & 0.01 & 0.00 \\
H-29 & -5.34 & -7.32 & 0.15 & 0.41 & $\mathbf{0 . 1 3}$ & 0.00 & 0.29 & 0.00 & 0.02 \\
H-33 & -5.57 & -7.55 & 0.00 & 0.04 & $\mathbf{0 . 9 3}$ & 0.00 & 0.02 & 0.00 & 0.00 \\
H-35 & -5.64 & -7.62 & 0.06 & 0.73 & $\mathbf{0 . 1 0}$ & 0.01 & 0.08 & 0.00 & 0.00 \\
H-36 & -5.71 & -7.69 & 0.00 & 0.02 & $\mathbf{0 . 9 7}$ & 0.00 & 0.00 & 0.00 & 0.00 \\
\hline
\end{tabular}

${ }^{a}$ Relative energy to LUMO. 
Table S24. MOs related to the $\pi$ and $\pi^{*}$ orbitals of bpy and HOMO in complexes $\left[\left\{\left(\mathrm{NH}_{3}\right)\left(\mathrm{PH}_{3}\right)\left(\mathrm{Ag}_{2} \mathrm{X}_{2}\right)\left(\mathrm{PPh}_{3}\right)\right\}\right.$-bpy- $\left.\left\{\left(\mathrm{PPh}_{3}\right)\left(\mathrm{Ag}_{2} \mathrm{X}_{2}\right)\left(\mathrm{NH}_{3}\right)\left(\mathrm{PH}_{3}\right)\right\}\right](\mathbf{3}-\mathbf{X})$ in DMSO.

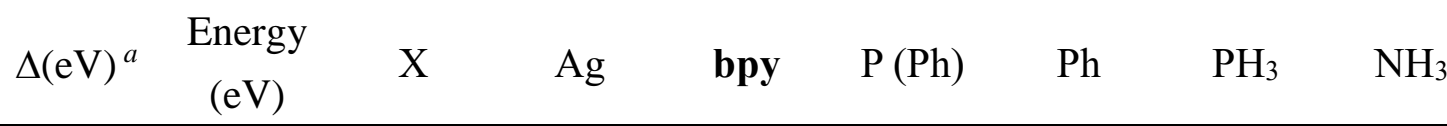

\begin{tabular}{lrllllllll}
\hline 3-I & & & & & & & & & \\
L+1 & 1.10 & -1.13 & 0.00 & 0.00 & $\mathbf{0 . 9 7}$ & 0.00 & 0.03 & 0.00 & 0.00 \\
LUMO & 0.00 & -2.23 & 0.00 & 0.00 & $\mathbf{0 . 9 9}$ & 0.00 & 0.00 & 0.00 & 0.00 \\
HOMO & -3.24 & -5.47 & $\mathbf{0 . 5 6}$ & $\mathbf{0 . 2 2}$ & $\mathbf{0 . 0 0}$ & 0.09 & 0.07 & 0.04 & 0.02 \\
H-28 & -5.38 & -7.61 & 0.02 & 0.21 & $\mathbf{0 . 7 2}$ & 0.00 & 0.03 & 0.02 & 0.00 \\
H-29 & -5.53 & -7.76 & 0.00 & 0.00 & $\mathbf{0 . 9 9}$ & 0.00 & 0.00 & 0.00 & 0.00 \\
H-30 & -5.67 & -7.90 & 0.00 & 0.00 & $\mathbf{0 . 9 9}$ & 0.00 & 0.00 & 0.00 & 0.00
\end{tabular}

\section{3-Br}

$\begin{array}{llllllllll}\text { L+1 } & 1.14 & -1.09 & 0.00 & 0.00 & \mathbf{0 . 9 7} & 0.00 & 0.03 & 0.00 & 0.00 \\ \text { LUMO } & 0.00 & -2.23 & 0.00 & 0.00 & \mathbf{0 . 9 9} & 0.00 & 0.00 & 0.00 & 0.00 \\ \text { HOMO } & -3.39 & -5.62 & \mathbf{0 . 4 5} & \mathbf{0 . 2 8} & \mathbf{0 . 0 0} & 0.12 & 0.09 & 0.04 & 0.02 \\ \text { H-26 } & -5.22 & -7.45 & 0.04 & 0.18 & \mathbf{0 . 0 6} & 0.00 & 0.69 & 0.00 & 0.00 \\ \text { H-27 } & -5.23 & -7.46 & 0.09 & 0.33 & \mathbf{0 . 1 0} & 0.01 & 0.44 & 0.00 & 0.02 \\ \text { H-28 } & -5.36 & -7.59 & 0.02 & 0.20 & \mathbf{0 . 7 3} & 0.00 & 0.02 & 0.02 & 0.00 \\ \text { H-29 } & -5.54 & -7.77 & 0.00 & 0.00 & \mathbf{0 . 9 9} & 0.00 & 0.00 & 0.00 & 0.00 \\ \text { H-30 } & -5.69 & -7.92 & 0.00 & 0.02 & \mathbf{0 . 9 6} & 0.00 & 0.00 & 0.00 & 0.00\end{array}$

\section{3-Cl}

\begin{tabular}{lrllllllll} 
L+1 & 1.13 & -1.06 & 0.00 & 0.00 & $\mathbf{0 . 9 4}$ & 0.00 & 0.05 & 0.00 & 0.00 \\
LUMO & 0.00 & -2.19 & 0.00 & 0.00 & $\mathbf{0 . 9 9}$ & 0.00 & 0.00 & 0.00 & 0.00 \\
HOMO & -3.57 & -5.76 & $\mathbf{0 . 2 3}$ & $\mathbf{0 . 4 1}$ & $\mathbf{0 . 0 1}$ & 0.03 & 0.03 & 0.12 & 0.16 \\
H-27 & -5.39 & -7.58 & 0.17 & 0.28 & $\mathbf{0 . 2 8}$ & 0.01 & 0.24 & 0.00 & 0.00 \\
H-28 & -5.41 & -7.60 & 0.06 & 0.22 & $\mathbf{0 . 5 9}$ & 0.00 & 0.11 & 0.00 & 0.00 \\
H-29 & -5.56 & -7.75 & 0.00 & 0.00 & $\mathbf{0 . 9 8}$ & 0.00 & 0.00 & 0.00 & 0.00 \\
H-30 & -5.67 & -7.86 & 0.00 & 0.03 & $\mathbf{0 . 9 6}$ & 0.00 & 0.00 & 0.00 & 0.00 \\
\hline
\end{tabular}

${ }^{a}$ Relative energy to LUMO. 
Table S25. Energy and components of molecular orbitals around Fermi energy for the model complex $\left[\left\{\left(\mathrm{NH}_{3}\right)\left(\mathrm{PH}_{3}\right)\left(\mathrm{Ag}_{2} \mathrm{Cl}_{2}\right)\left(\mathrm{PPh}_{3}\right)\right\}\right.$-bpy- $\left.\left\{\left(\mathrm{PPh}_{3}\right)\left(\mathrm{Ag}_{2} \mathrm{Cl}_{2}\right)\left(\mathrm{NH}_{3}\right)\left(\mathrm{PH}_{3}\right)\right\}\right]$ with planar bpy (4-Cl) in DMSO.

\begin{tabular}{|c|c|c|c|c|c|c|c|c|}
\hline & $\begin{array}{c}\text { Energy } \\
(\mathrm{eV})\end{array}$ & $\mathrm{Cl}$ & $\mathrm{Ag}$ & bpy & $\mathrm{P}(\mathrm{Ph})$ & $\mathrm{Ph}$ & $\mathrm{PH}_{3}$ & $\mathrm{NH}_{3}$ \\
\hline$L+10$ & -0.45 & 0.00 & 0.14 & 0.06 & 0.00 & 0.77 & 0.00 & 0.01 \\
\hline $\mathrm{L}+9$ & -0.45 & 0.00 & 0.15 & 0.02 & 0.00 & 0.81 & 0.00 & 0.01 \\
\hline $\mathrm{L}+8$ & -0.53 & 0.00 & 0.02 & 0.89 & 0.00 & 0.10 & 0.00 & 0.00 \\
\hline $\mathrm{L}+7$ & -0.71 & 0.00 & 0.03 & 0.00 & 0.04 & 0.92 & 0.00 & 0.00 \\
\hline $\mathrm{L}+6$ & -0.74 & 0.00 & 0.03 & 0.00 & 0.05 & 0.91 & 0.00 & 0.00 \\
\hline $\mathrm{L}+5$ & -0.79 & 0.00 & 0.02 & 0.00 & 0.05 & 0.91 & 0.00 & 0.00 \\
\hline $\mathrm{L}+4$ & -0.81 & 0.00 & 0.04 & 0.00 & 0.05 & 0.90 & 0.00 & 0.00 \\
\hline $\mathrm{L}+3$ & -0.82 & 0.00 & 0.02 & 0.00 & 0.06 & 0.92 & 0.00 & 0.00 \\
\hline $\mathrm{L}+2$ & -0.83 & 0.00 & 0.00 & 0.02 & 0.07 & 0.90 & 0.00 & 0.00 \\
\hline $\mathrm{L}+1$ & -1.14 & 0.00 & 0.00 & 0.98 & 0.00 & 0.01 & 0.00 & 0.00 \\
\hline LUMO & -2.26 & 0.00 & 0.00 & 0.99 & 0.00 & 0.00 & 0.00 & 0.00 \\
\hline HOMO & -5.76 & 0.23 & 0.41 & 0.00 & 0.03 & 0.03 & 0.12 & 0.16 \\
\hline H-1 & -5.78 & 0.32 & 0.35 & 0.00 & 0.12 & 0.12 & 0.02 & 0.07 \\
\hline $\mathrm{H}-2$ & -5.85 & 0.23 & 0.37 & 0.00 & 0.16 & 0.15 & 0.00 & 0.08 \\
\hline H-3 & -5.92 & 0.23 & 0.40 & 0.00 & 0.05 & 0.06 & 0.07 & 0.19 \\
\hline $\mathrm{H}-4$ & -6.21 & 0.58 & 0.34 & 0.00 & 0.02 & 0.03 & 0.01 & 0.01 \\
\hline H-5 & -6.22 & 0.60 & 0.35 & 0.00 & 0.01 & 0.03 & 0.00 & 0.00 \\
\hline H-6 & -6.28 & 0.23 & 0.41 & 0.12 & 0.03 & 0.07 & 0.14 & 0.00 \\
\hline H-7 & -6.32 & 0.12 & 0.39 & 0.10 & 0.07 & 0.15 & 0.15 & 0.01 \\
\hline H-8 & -6.51 & 0.34 & 0.38 & 0.16 & 0.00 & 0.03 & 0.08 & 0.00 \\
\hline H-9 & -6.65 & 0.47 & 0.33 & 0.09 & 0.01 & 0.06 & 0.03 & 0.01 \\
\hline $\mathrm{H}-10$ & -6.73 & 0.51 & 0.39 & 0.05 & 0.00 & 0.04 & 0.00 & 0.00 \\
\hline H-11 & -6.74 & 0.80 & 0.18 & 0.00 & 0.00 & 0.00 & 0.00 & 0.00 \\
\hline $\mathrm{H}-12$ & -6.82 & 0.65 & 0.22 & 0.02 & 0.00 & 0.07 & 0.02 & 0.00 \\
\hline H-13 & -6.84 & 0.62 & 0.32 & 0.00 & 0.00 & 0.04 & 0.00 & 0.00 \\
\hline H-14 & -7.13 & 0.00 & 0.00 & 0.00 & 0.00 & 0.99 & 0.00 & 0.00 \\
\hline H-15 & -7.14 & 0.02 & 0.02 & 0.00 & 0.00 & 0.95 & 0.00 & 0.00 \\
\hline H-16 & -7.17 & 0.04 & 0.03 & 0.00 & 0.00 & 0.92 & 0.00 & 0.00 \\
\hline H-17 & -7.18 & 0.01 & 0.00 & 0.00 & 0.00 & 0.97 & 0.00 & 0.00 \\
\hline H-18 & -7.23 & 0.41 & 0.17 & 0.02 & 0.00 & 0.33 & 0.05 & 0.02 \\
\hline H-19 & -7.27 & 0.26 & 0.14 & 0.01 & 0.00 & 0.53 & 0.04 & 0.00 \\
\hline H-20 & -7.36 & 0.01 & 0.03 & 0.03 & 0.00 & 0.92 & 0.00 & 0.00 \\
\hline
\end{tabular}


(Continued)

\begin{tabular}{lllllllll} 
H-21 & -7.37 & 0.00 & 0.00 & 0.00 & 0.00 & 0.98 & 0.00 & 0.00 \\
H-22 & -7.38 & 0.03 & 0.07 & 0.02 & 0.00 & 0.87 & 0.00 & 0.00 \\
H-23 & -7.38 & 0.04 & 0.03 & 0.00 & 0.00 & 0.91 & 0.00 & 0.00 \\
H-24 & -7.42 & 0.09 & 0.04 & 0.01 & 0.00 & 0.84 & 0.00 & 0.00 \\
H-25 & -7.46 & 0.06 & 0.02 & 0.00 & 0.00 & 0.90 & 0.00 & 0.00 \\
H-26 & -7.53 & 0.09 & 0.23 & 0.21 & 0.01 & 0.44 & 0.00 & 0.00 \\
H-27 & -7.55 & 0.09 & 0.22 & 0.54 & 0.00 & 0.13 & 0.01 & 0.00 \\
H-28 & -7.59 & 0.14 & 0.33 & 0.22 & 0.02 & 0.27 & 0.00 & 0.01 \\
H-29 & -7.73 & 0.00 & 0.00 & 0.99 & 0.00 & 0.00 & 0.00 & 0.00 \\
H-30 & -7.89 & 0.15 & 0.55 & 0.24 & 0.00 & 0.03 & 0.00 & 0.01 \\
H-31 & -7.90 & 0.05 & 0.16 & 0.77 & 0.00 & 0.01 & 0.00 & 0.00 \\
H-32 & -8.02 & 0.15 & 0.66 & 0.02 & 0.04 & 0.12 & 0.00 & 0.00 \\
H-33 & -8.06 & 0.07 & 0.84 & 0.00 & 0.01 & 0.04 & 0.02 & 0.01 \\
H-34 & -8.07 & 0.19 & 0.72 & 0.01 & 0.00 & 0.04 & 0.02 & 0.02 \\
H-35 & -8.11 & 0.09 & 0.84 & 0.02 & 0.00 & 0.02 & 0.01 & 0.01 \\
H-36 & -8.18 & 0.13 & 0.73 & 0.05 & 0.02 & 0.05 & 0.01 & 0.01 \\
H-37 & -8.41 & 0.14 & 0.79 & 0.03 & 0.00 & 0.03 & 0.00 & 0.00 \\
H-38 & -8.47 & 0.12 & 0.78 & 0.06 & 0.00 & 0.03 & 0.00 & 0.00 \\
H-39 & -8.69 & 0.32 & 0.51 & 0.12 & 0.00 & 0.00 & 0.02 & 0.00 \\
H-40 & -8.72 & 0.25 & 0.70 & 0.00 & 0.00 & 0.02 & 0.00 & 0.00 \\
\hline & & & & & & & &
\end{tabular}


Table S26. Energy and major components of excited states for the model complex $\left[\left\{\left(\mathrm{NH}_{3}\right)\left(\mathrm{PH}_{3}\right)\left(\mathrm{Ag}_{2} \mathrm{Cl}_{2}\right)\left(\mathrm{PPh}_{3}\right)\right\}\right.$-bpy- $\left.\left\{\left(\mathrm{PPh}_{3}\right)\left(\mathrm{Ag}_{2} \mathrm{Cl}_{2}\right)\left(\mathrm{NH}_{3}\right)\left(\mathrm{PH}_{3}\right)\right\}\right]$ with planar bpy (4-Cl) in DMSO.

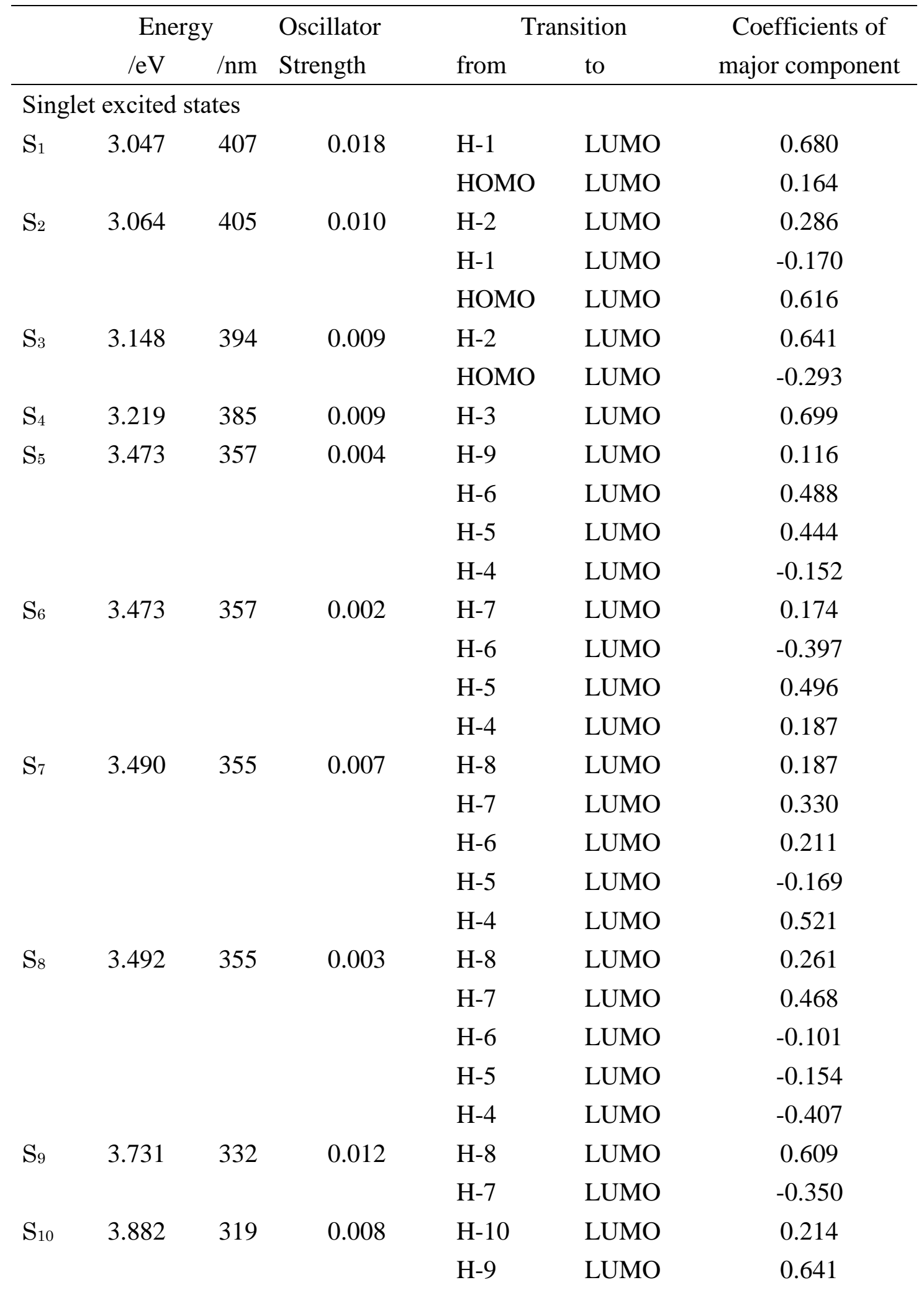




\section{(Continued)}

\begin{tabular}{|c|c|c|c|c|c|c|}
\hline & & & & H-6 & LUMO & -0.185 \\
\hline \multirow[t]{3}{*}{$\mathrm{S}_{11}$} & 3.989 & 311 & 0.019 & H-11 & LUMO & -0.468 \\
\hline & & & & $\mathrm{H}-10$ & LUMO & 0.494 \\
\hline & & & & $\mathrm{H}-9$ & LUMO & -0.168 \\
\hline \multirow[t]{3}{*}{$\mathrm{S}_{12}$} & 3.993 & 311 & 0.003 & $\mathrm{H}-11$ & LUMO & 0.526 \\
\hline & & & & H-10 & LUMO & 0.436 \\
\hline & & & & H-9 & LUMO & -0.159 \\
\hline \multirow[t]{2}{*}{$\mathrm{S}_{13}$} & 4.088 & 303 & 0.006 & H-13 & LUMO & 0.209 \\
\hline & & & & H-12 & LUMO & 0.665 \\
\hline \multirow[t]{2}{*}{$\mathrm{S}_{14}$} & 4.091 & 303 & 0.005 & $\mathrm{H}-13$ & LUMO & 0.671 \\
\hline & & & & $\mathrm{H}-12$ & LUMO & -0.206 \\
\hline \multirow[t]{4}{*}{$\mathrm{S}_{15}$} & 4.163 & 298 & 0.002 & H-2 & $\mathrm{L}+1$ & 0.235 \\
\hline & & & & H-1 & $\mathrm{L}+1$ & 0.408 \\
\hline & & & & HOMO & $\mathrm{L}+1$ & 0.491 \\
\hline & & & & HOMO & LUMO & 0.203 \\
\hline
\end{tabular}

Triplet excited states

\begin{tabular}{|c|c|c|c|c|c|c|}
\hline \multirow[t]{5}{*}{$\mathrm{T}_{1}$} & 2.996 & 414 & 0.000 & $\mathrm{H}-27$ & LUMO & -0.131 \\
\hline & & & & H-6 & LUMO & -0.175 \\
\hline & & & & $\mathrm{H}-2$ & LUMO & 0.331 \\
\hline & & & & $\mathrm{H}-1$ & LUMO & 0.249 \\
\hline & & & & HOMO & LUMO & 0.495 \\
\hline \multirow[t]{3}{*}{$\mathrm{T}_{2}$} & 3.031 & 409 & 0.000 & $\mathrm{H}-2$ & LUMO & -0.141 \\
\hline & & & & H-1 & LUMO & 0.633 \\
\hline & & & & HOMO & LUMO & -0.216 \\
\hline \multirow[t]{3}{*}{$\mathrm{T}_{3}$} & 3.134 & 396 & 0.000 & $\mathrm{H}-3$ & LUMO & 0.135 \\
\hline & & & & $\mathrm{H}-2$ & LUMO & 0.536 \\
\hline & & & & HOMO & LUMO & -0.399 \\
\hline \multirow[t]{4}{*}{$\mathrm{T}_{4}$} & 3.170 & 391 & 0.000 & H-8 & LUMO & -0.173 \\
\hline & & & & $\mathrm{H}-7$ & LUMO & -0.261 \\
\hline & & & & $\mathrm{H}-3$ & LUMO & 0.555 \\
\hline & & & & $\mathrm{H}-2$ & LUMO & -0.198 \\
\hline \multirow[t]{4}{*}{$\mathrm{T}_{5}$} & 3.291 & 377 & 0.000 & $\mathrm{H}-28$ & LUMO & 0.112 \\
\hline & & & & $\mathrm{H}-27$ & LUMO & -0.197 \\
\hline & & & & H-8 & LUMO & 0.389 \\
\hline & & & & $\mathrm{H}-7$ & LUMO & 0.384 \\
\hline
\end{tabular}


(Continued)

\begin{tabular}{|c|c|c|c|c|c|c|}
\hline & & & & H-6 & LUMO & 0.121 \\
\hline & & & & $\mathrm{H}-3$ & LUMO & 0.213 \\
\hline & & & & $\mathrm{H}-1$ & LUMO & -0.139 \\
\hline \multirow[t]{8}{*}{$\mathrm{T}_{6}$} & 3.311 & 375 & 0.000 & $\mathrm{H}-28$ & LUMO & 0.138 \\
\hline & & & & $\mathrm{H}-10$ & LUMO & 0.151 \\
\hline & & & & H-9 & LUMO & 0.208 \\
\hline & & & & H-7 & LUMO & -0.180 \\
\hline & & & & H-6 & LUMO & 0.469 \\
\hline & & & & $\mathrm{H}-4$ & LUMO & -0.105 \\
\hline & & & & $\mathrm{H}-3$ & LUMO & -0.230 \\
\hline & & & & HOMO & LUMO & 0.156 \\
\hline \multirow[t]{9}{*}{$\mathrm{T}_{7}$} & 3.335 & 372 & 0.000 & $\mathrm{H}-31$ & $\mathrm{~L}+8$ & 0.101 \\
\hline & & & & H-29 & $\mathrm{L}+1$ & 0.149 \\
\hline & & & & H-28 & LUMO & -0.151 \\
\hline & & & & $\mathrm{H}-27$ & LUMO & 0.344 \\
\hline & & & & H-26 & LUMO & 0.197 \\
\hline & & & & $\mathrm{H}-7$ & LUMO & 0.139 \\
\hline & & & & H-6 & LUMO & 0.306 \\
\hline & & & & $\mathrm{H}-3$ & LUMO & 0.243 \\
\hline & & & & $\mathrm{H}-2$ & LUMO & 0.188 \\
\hline $\mathrm{T}_{8}$ & 3.473 & 357 & 0.000 & $\mathrm{H}-5$ & LUMO & 0.696 \\
\hline $\mathrm{T}_{9}$ & 3.489 & 355 & 0.000 & $\mathrm{H}-4$ & LUMO & 0.689 \\
\hline \multirow[t]{12}{*}{$\mathrm{T}_{10}$} & 3.713 & 334 & 0.000 & H-24 & $\mathrm{L}+2$ & 0.101 \\
\hline & & & & H-22 & $\mathrm{L}+2$ & -0.165 \\
\hline & & & & $\mathrm{H}-22$ & $\mathrm{~L}+7$ & -0.166 \\
\hline & & & & $\mathrm{H}-21$ & $\mathrm{~L}+2$ & -0.132 \\
\hline & & & & $\mathrm{H}-21$ & $\mathrm{~L}+13$ & -0.118 \\
\hline & & & & H-18 & $\mathrm{L}+2$ & 0.154 \\
\hline & & & & $\mathrm{H}-17$ & $\mathrm{~L}+4$ & 0.110 \\
\hline & & & & H-8 & LUMO & -0.113 \\
\hline & & & & $\mathrm{H}-7$ & $\mathrm{~L}+2$ & 0.200 \\
\hline & & & & H-3 & $\mathrm{L}+2$ & -0.187 \\
\hline & & & & H-1 & $\mathrm{L}+2$ & -0.302 \\
\hline & & & & H-1 & $\mathrm{L}+7$ & -0.111 \\
\hline $\mathrm{T}_{11}$ & 3.718 & 333 & 0.000 & $\mathrm{H}-8$ & LUMO & 0.493 \\
\hline
\end{tabular}


(Continued)

\begin{tabular}{|c|c|c|c|c|c|c|}
\hline & & & & $\mathrm{H}-7$ & LUMO & -0.431 \\
\hline \multirow[t]{14}{*}{$\mathrm{T}_{12}$} & 3.738 & 332 & 0.000 & H-25 & $\mathrm{L}+6$ & 0.120 \\
\hline & & & & $\mathrm{H}-23$ & $\mathrm{~L}+16$ & 0.110 \\
\hline & & & & H-20 & $\mathrm{L}+3$ & -0.153 \\
\hline & & & & $\mathrm{H}-20$ & $\mathrm{~L}+5$ & 0.161 \\
\hline & & & & $\mathrm{H}-20$ & $\mathrm{~L}+10$ & -0.144 \\
\hline & & & & H-19 & $\mathrm{L}+16$ & 0.103 \\
\hline & & & & H-16 & $\mathrm{L}+3$ & -0.108 \\
\hline & & & & H-16 & $\mathrm{L}+5$ & -0.141 \\
\hline & & & & $\mathrm{H}-15$ & $\mathrm{~L}+12$ & 0.125 \\
\hline & & & & H-6 & $\mathrm{L}+3$ & -0.150 \\
\hline & & & & $\mathrm{H}-2$ & $\mathrm{~L}+3$ & 0.330 \\
\hline & & & & $\mathrm{H}-2$ & $\mathrm{~L}+5$ & -0.112 \\
\hline & & & & $\mathrm{H}-2$ & $\mathrm{~L}+6$ & -0.121 \\
\hline & & & & HOMO & $\mathrm{L}+3$ & 0.160 \\
\hline \multirow[t]{14}{*}{$\mathrm{T}_{13}$} & 3.744 & 331 & 0.000 & $\mathrm{H}-24$ & $\mathrm{~L}+2$ & -0.104 \\
\hline & & & & $\mathrm{H}-24$ & $\mathrm{~L}+4$ & 0.151 \\
\hline & & & & $\mathrm{H}-24$ & $\mathrm{~L}+7$ & -0.101 \\
\hline & & & & $\mathrm{H}-24$ & $\mathrm{~L}+9$ & 0.126 \\
\hline & & & & H-21 & $\mathrm{L}+4$ & -0.134 \\
\hline & & & & H-21 & $\mathrm{L}+11$ & 0.141 \\
\hline & & & & H-17 & $\mathrm{L}+4$ & 0.130 \\
\hline & & & & H-17 & $\mathrm{L}+11$ & -0.175 \\
\hline & & & & H-14 & $\mathrm{L}+7$ & -0.118 \\
\hline & & & & H-14 & $\mathrm{L}+9$ & 0.108 \\
\hline & & & & H-7 & $\mathrm{L}+4$ & -0.171 \\
\hline & & & & $\mathrm{H}-3$ & $\mathrm{~L}+4$ & 0.171 \\
\hline & & & & $\mathrm{H}-1$ & $\mathrm{~L}+4$ & 0.283 \\
\hline & & & & H-1 & $\mathrm{L}+7$ & -0.110 \\
\hline \multirow[t]{6}{*}{$\mathrm{T}_{14}$} & 3.766 & 329 & 0.000 & $\mathrm{H}-23$ & $\mathrm{~L}+12$ & -0.166 \\
\hline & & & & H-20 & $\mathrm{L}+3$ & 0.193 \\
\hline & & & & H-19 & $\mathrm{L}+5$ & -0.114 \\
\hline & & & & H-16 & $L+16$ & 0.110 \\
\hline & & & & $\mathrm{H}-15$ & $\mathrm{~L}+5$ & 0.140 \\
\hline & & & & $\mathrm{H}-15$ & $\mathrm{~L}+6$ & 0.154 \\
\hline
\end{tabular}




\section{(Continued)}

\begin{tabular}{|c|c|c|c|c|c|c|}
\hline & & & & $\mathrm{H}-15$ & $L+16$ & -0.109 \\
\hline & & & & H-6 & $\mathrm{L}+5$ & -0.128 \\
\hline & & & & $\mathrm{H}-2$ & $\mathrm{~L}+5$ & 0.274 \\
\hline & & & & $\mathrm{H}-2$ & $\mathrm{~L}+6$ & -0.161 \\
\hline & & & & HOMO & $\mathrm{L}+5$ & 0.129 \\
\hline \multirow[t]{20}{*}{$\mathrm{T}_{15}$} & 3.814 & 325 & 0.000 & $\mathrm{H}-25$ & $\mathrm{~L}+3$ & 0.116 \\
\hline & & & & $\mathrm{H}-25$ & $\mathrm{~L}+5$ & -0.204 \\
\hline & & & & $\mathrm{H}-25$ & $\mathrm{~L}+6$ & 0.103 \\
\hline & & & & H-25 & $\mathrm{L}+10$ & -0.113 \\
\hline & & & & $\mathrm{H}-23$ & $\mathrm{~L}+3$ & -0.139 \\
\hline & & & & $\mathrm{H}-23$ & $\mathrm{~L}+6$ & -0.133 \\
\hline & & & & $\mathrm{H}-20$ & $\mathrm{~L}+6$ & -0.172 \\
\hline & & & & $\mathrm{H}-20$ & $\mathrm{~L}+12$ & 0.116 \\
\hline & & & & H-16 & $\mathrm{L}+5$ & -0.128 \\
\hline & & & & H-16 & $\mathrm{L}+12$ & 0.117 \\
\hline & & & & H-16 & $L+16$ & 0.101 \\
\hline & & & & $\mathrm{H}-15$ & $\mathrm{~L}+3$ & -0.157 \\
\hline & & & & H-15 & $\mathrm{L}+10$ & -0.113 \\
\hline & & & & H-15 & $\mathrm{L}+12$ & 0.194 \\
\hline & & & & $\mathrm{H}-2$ & $\mathrm{~L}+6$ & 0.184 \\
\hline & & & & $\mathrm{H}-2$ & $\mathrm{~L}+10$ & -0.156 \\
\hline & & & & H-6 & LUMO & -0.190 \\
\hline & & & & $\mathrm{H}-2$ & LUMO & 0.307 \\
\hline & & & & H-1 & LUMO & 0.247 \\
\hline & & & & HOMO & LUMO & 0.509 \\
\hline
\end{tabular}


a)

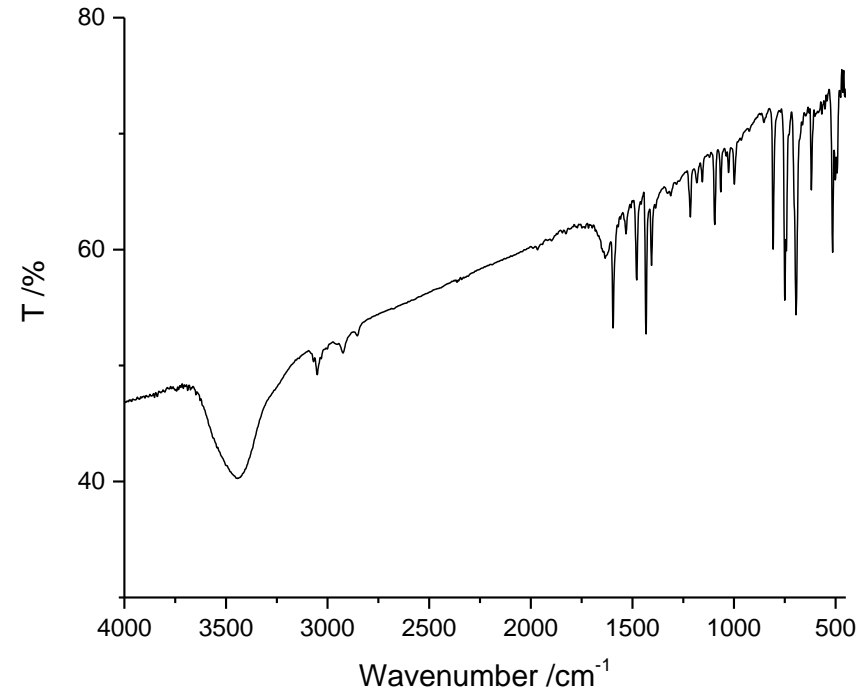

b)

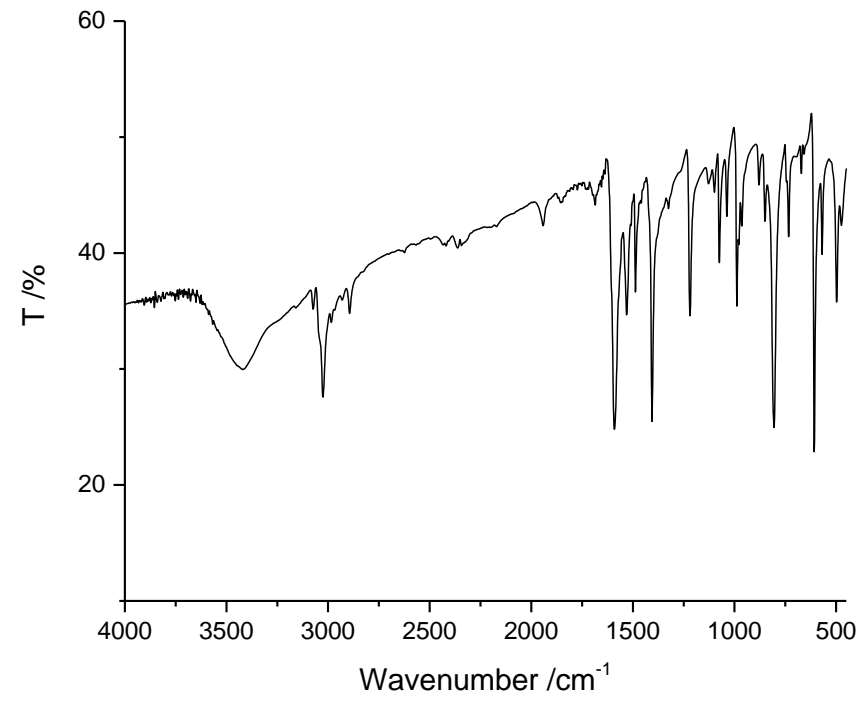

c)

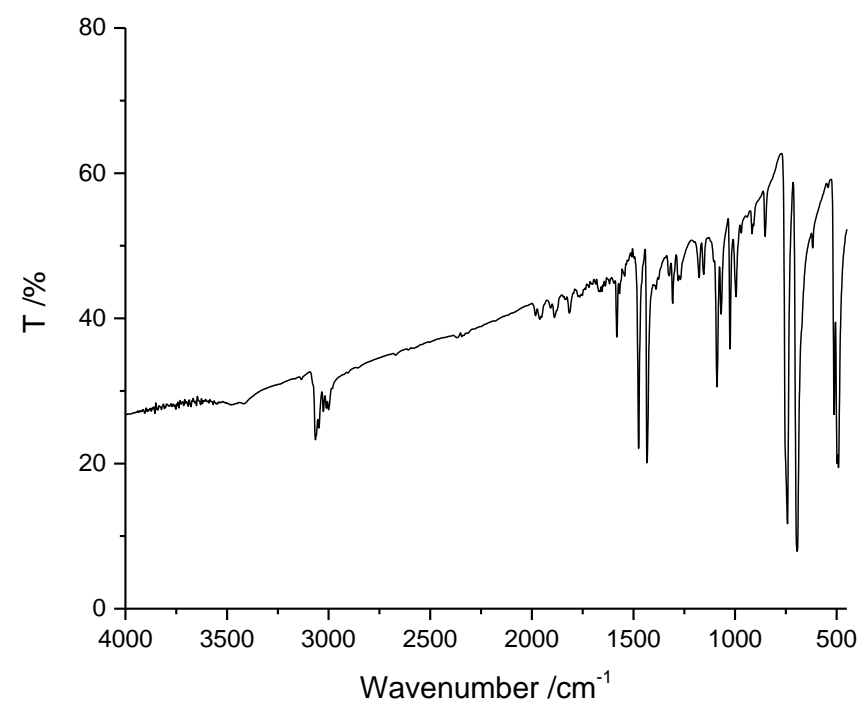

Figure S1. The IR absorption spectrum of $\left[\mathrm{Ag}_{2} \mathrm{I}_{2}\left(\mathrm{PPh}_{3}\right)_{2}\right.$ (bpy) $]$ (a) together with that of bpy (b) and $\mathrm{PPh}_{3}$ (c). 

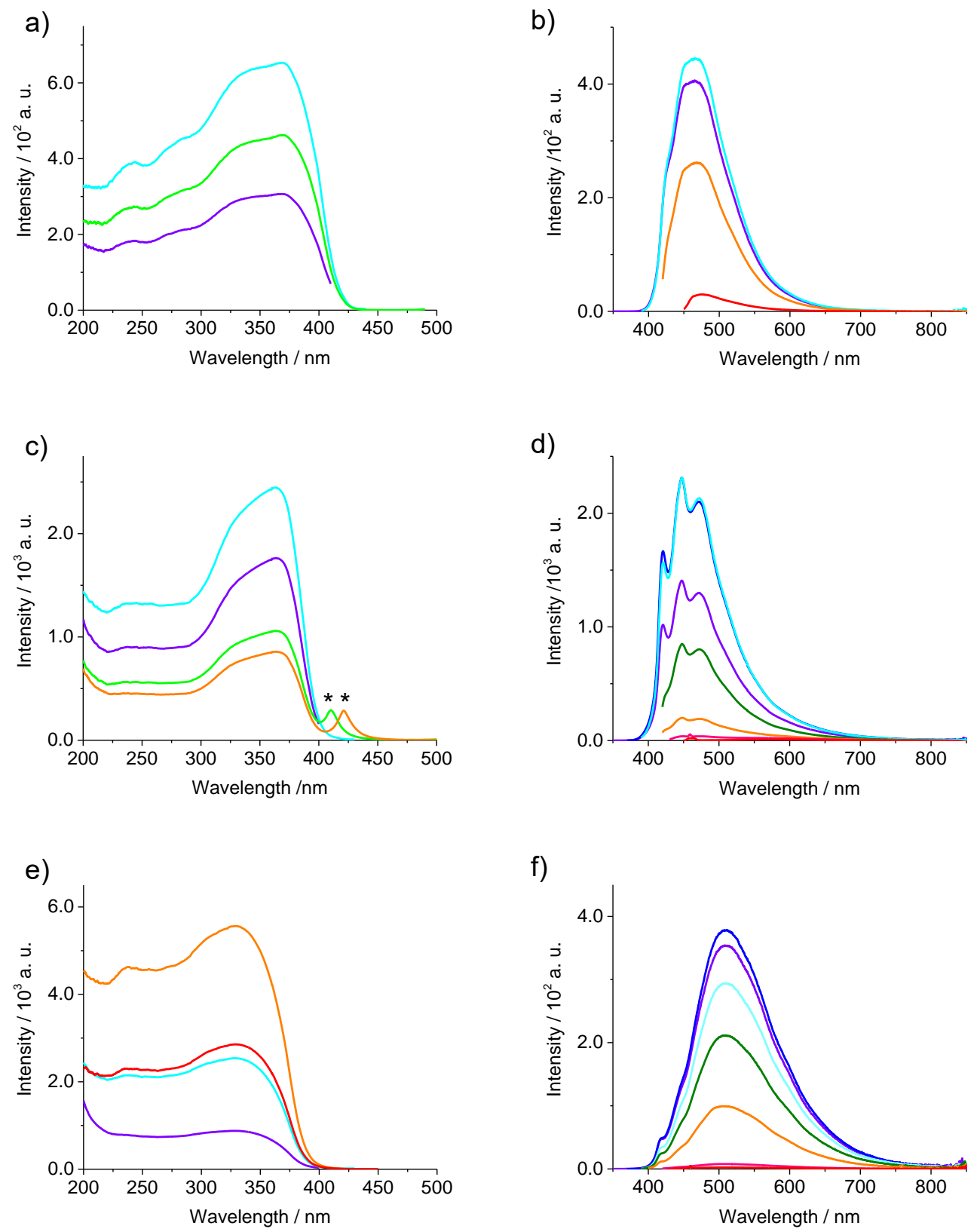

Figure S2. Dependence of excitation (a, c, e) and emission spectra (b, d, f) of $\left[\mathrm{Ag}_{2} \mathrm{X}_{2}\left(\mathrm{PPh}_{3}\right)_{2}(\mathrm{bpy})\right]_{n}$ upon $\lambda_{\mathrm{em}}$ and $\lambda_{\text {ex. }}$ a) $\mathrm{X}=\mathrm{I}$, with $\lambda_{\mathrm{em}}$ at $420 \mathrm{~nm}$ (violet), $450 \mathrm{~nm}$ (sky blue), $500 \mathrm{~nm}$ (green); b) X = I, with $\lambda_{\text {ex }}$ at $330 \mathrm{~nm}$ (violet), $360 \mathrm{~nm}$ (sky blue), $400 \mathrm{~nm}$ (orange), $420 \mathrm{~nm}$ (red); c) $\mathrm{X}=\mathrm{Br}$, with $\lambda_{\text {em }}$ at $420 \mathrm{~nm}$ (violet), $450 \mathrm{~nm}$ (sky blue), $520 \mathrm{~nm}$ (green), $530 \mathrm{~nm}$ (orange); d) $\mathrm{X}=\mathrm{Br}$, with $\lambda_{\text {ex }}$ at $310 \mathrm{~nm}$ (violet), 350 $\mathrm{nm}$ (blue), $370 \mathrm{~nm}$ (sky blue), $390 \mathrm{~nm}$ (green), $400 \mathrm{~nm}$ (orange), $410 \mathrm{~nm}$ (pink), 430 $\mathrm{nm}$ (red); e) $\mathrm{X}=\mathrm{Cl}$, with $\lambda_{\mathrm{em}}$ at $420 \mathrm{~nm}$ (violet), $450 \mathrm{~nm}$ (sky blue), $550 \mathrm{~nm}$ (orange), $600 \mathrm{~nm}$ (red); f) $\mathrm{X}=\mathrm{Cl}$ with $\lambda_{\text {ex }}$ at $300 \mathrm{~nm}$ (violet), $340 \mathrm{~nm}$ (blue), $360 \mathrm{~nm}$ (sky blue), $370 \mathrm{~nm}$ (green), $380 \mathrm{~nm}$ (orange), $400 \mathrm{~nm}$ (pink), $410 \mathrm{~nm}$ (red). (*) Artifact. 
a)

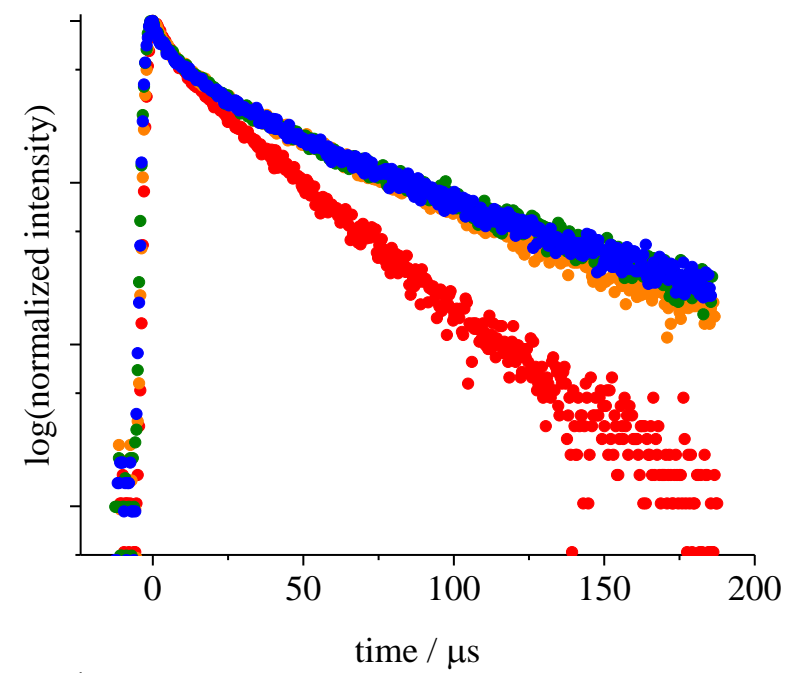

b)

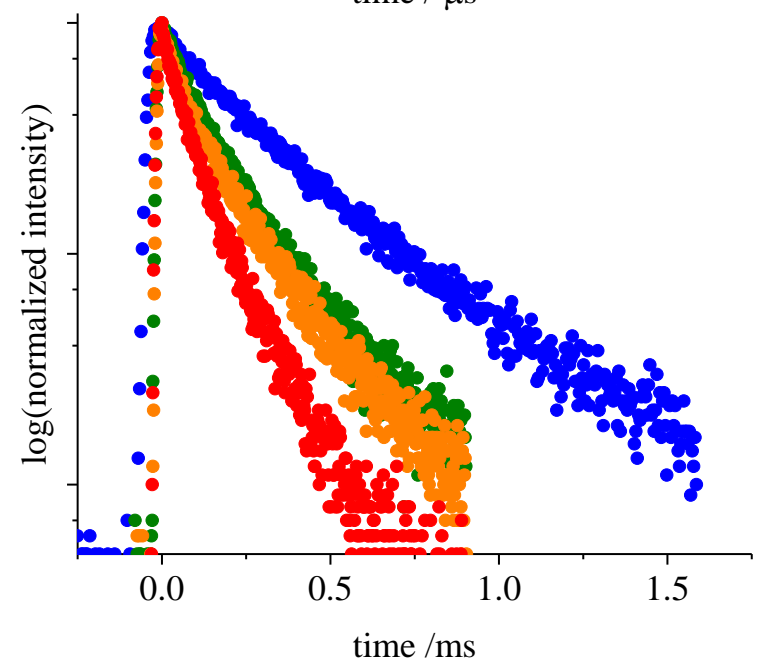

c)

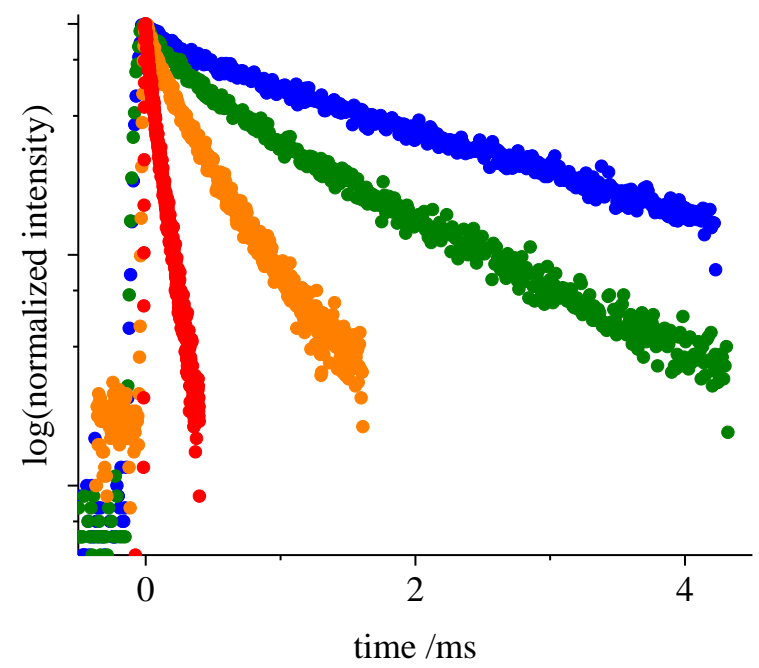

Figure S3. Emission decays of $\left[\mathrm{Ag}_{2} \mathrm{X}_{2}\left(\mathrm{PPh}_{3}\right)_{2}(\mathrm{bpy})\right]$ at temperatures of $80 \mathrm{~K}$ (blue), $170 \mathrm{~K}$ (green), $230 \mathrm{~K}$ (orange), and $290 \mathrm{~K}$ (red); a): $\mathrm{X}=\mathrm{I}, \mathrm{b}$ ): $\mathrm{X}=\mathrm{Br}, \mathrm{c}$ ): $\mathrm{X}=\mathrm{Cl}$. 


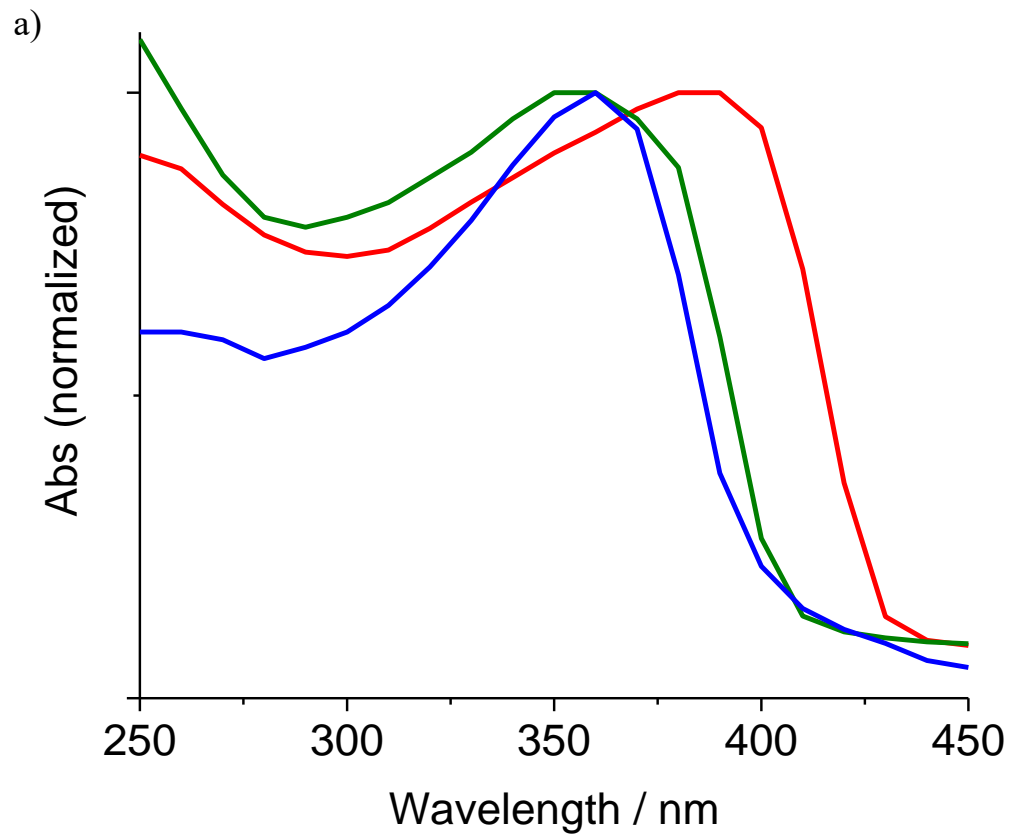

b)

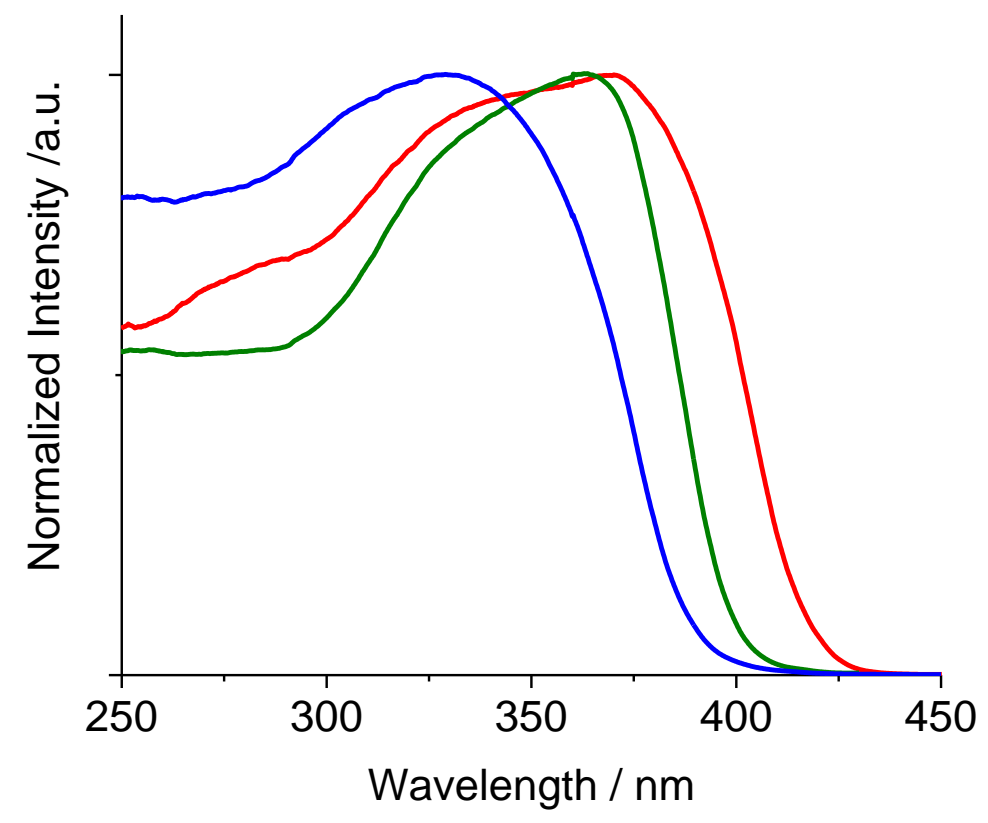

Figure S4. Absorption (a) and excitation (b) spectra of $\left[\mathrm{Ag}_{2} \mathrm{X}_{2}\left(\mathrm{PPh}_{3}\right)_{2}(\mu \text {-bpy })\right]_{n}$ in the solid state at room temperature; X: I (red), X: Br (green), X: Cl (blue). 


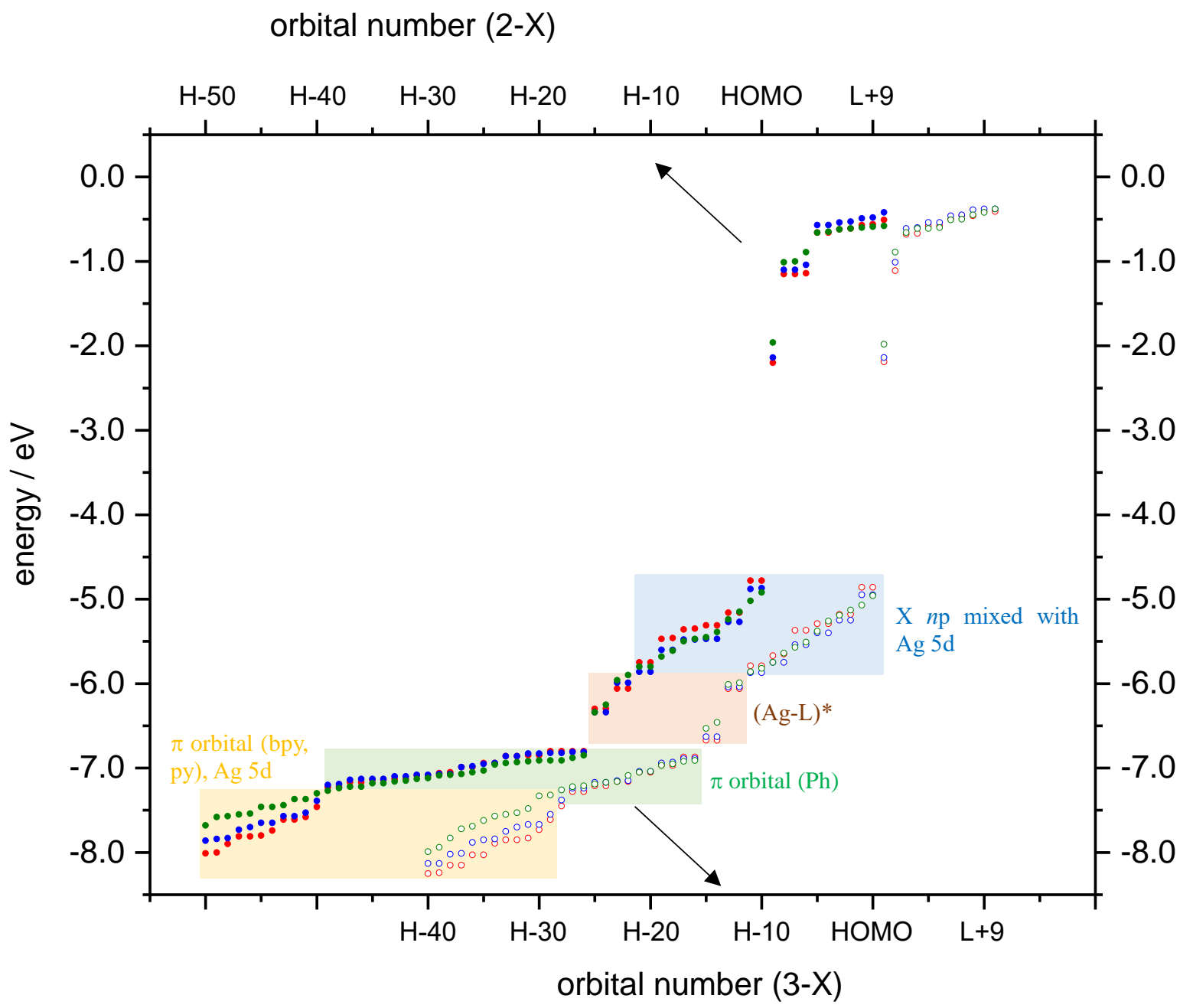

Figure S5. Energy of MOs of 2-X (filled circle) and 3-X (open circle). $\mathrm{X}=\mathrm{I}$ : red; $\mathrm{X}=\mathrm{Br}$ : green; $\mathrm{X}=\mathrm{Cl}$ : blue. 
a)

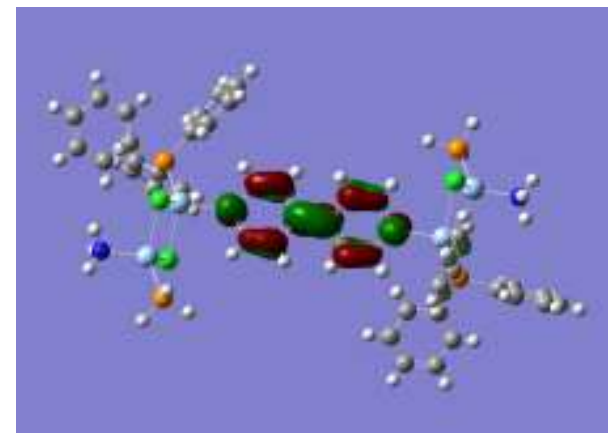

b)

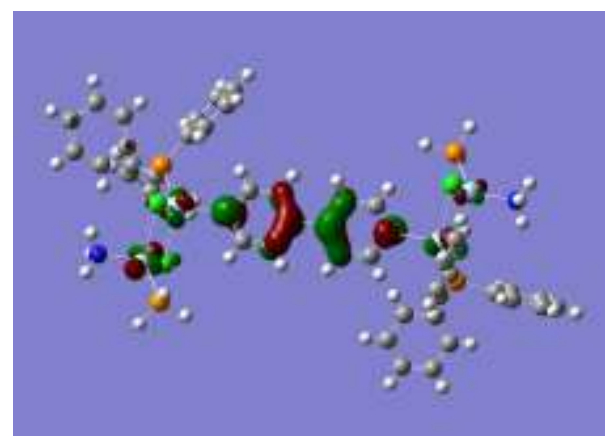

c)

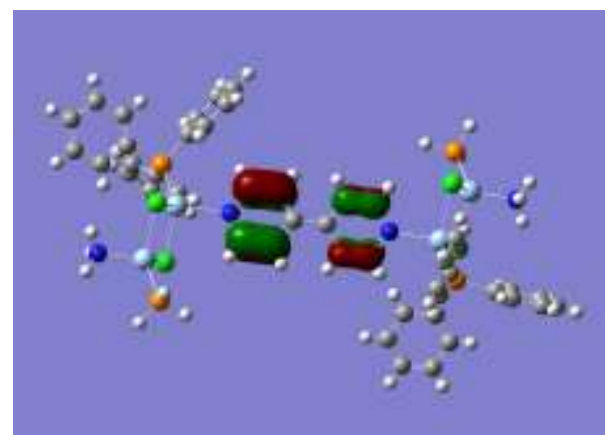

d)

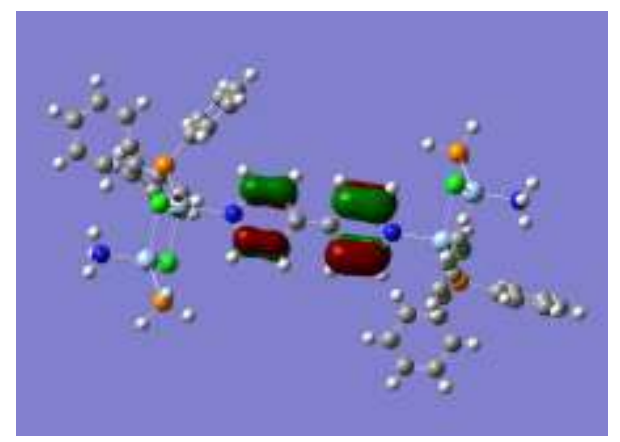

Figure S6. Surface diagrams of MOs corresponding to $\pi$ orbitals of bpy in 3-Cl in DMSO with an isovalue of 0.04. a): LUMO, b): H-28, c): H-29, d): H-30. 


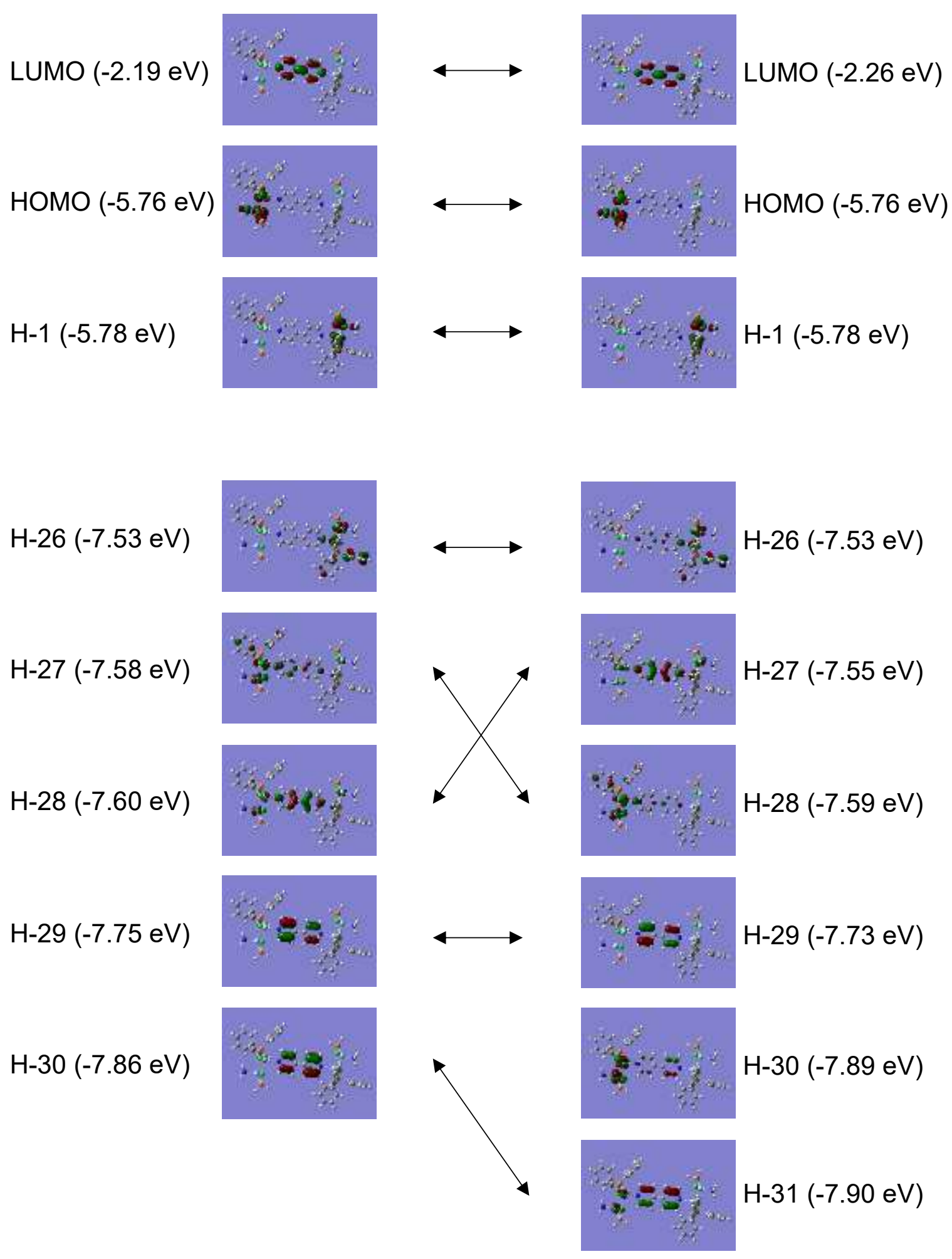

Figure S7. Selected surface diagrams of MOs of 3-Cl (left) and 4-Cl (right) with an isovalue of 0.04 . 
a)

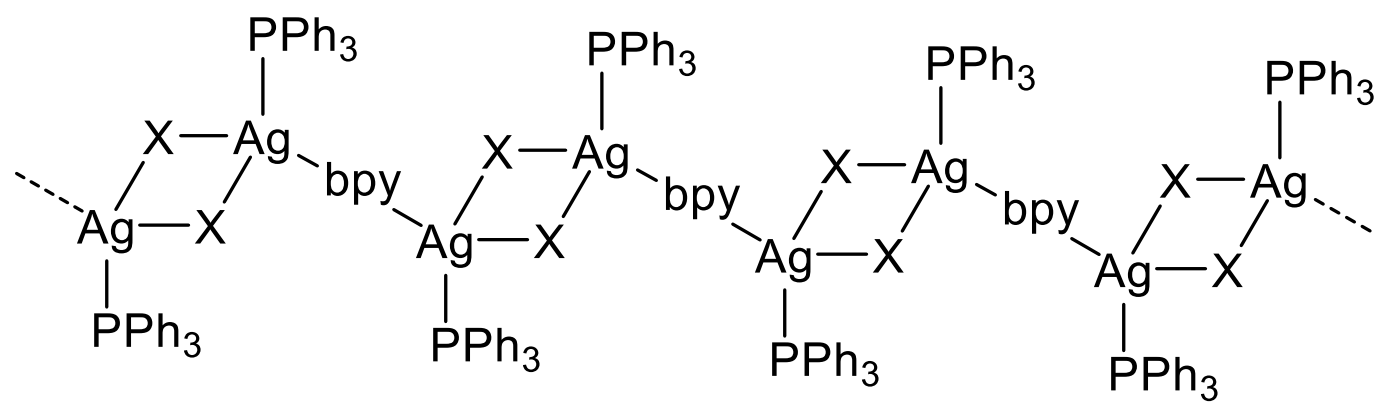

b)

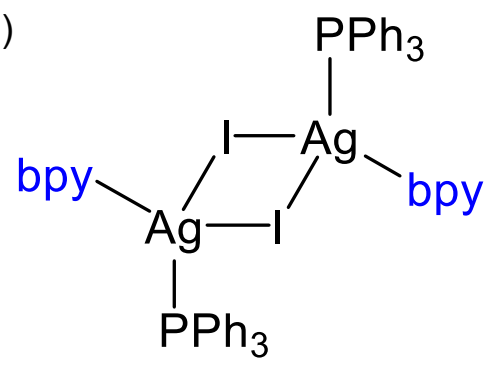

c)

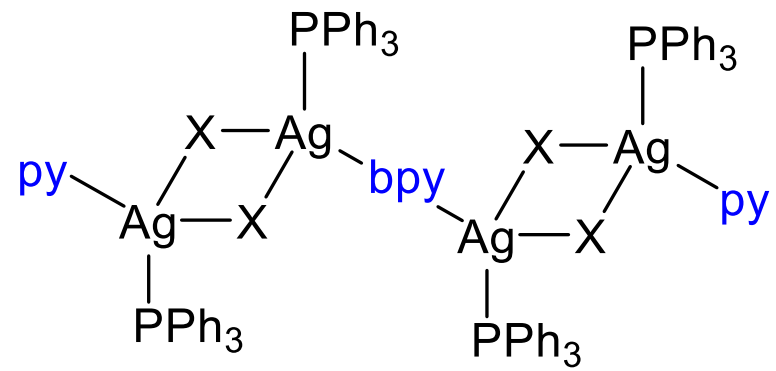

d)

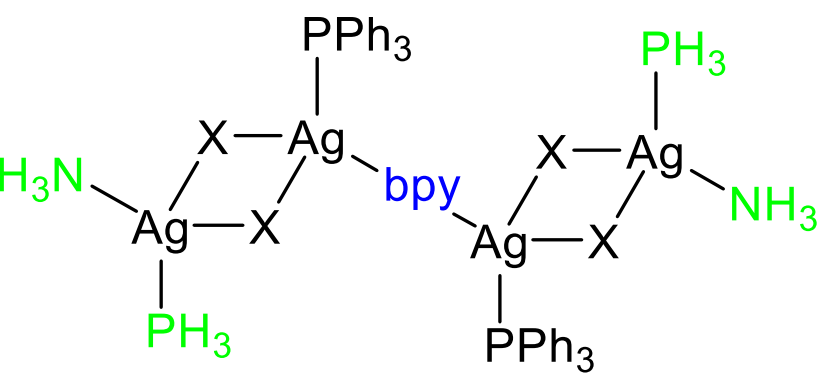

Scheme S1. Schematic diagram of the model complexes used in the calculations together with that of $\left[\mathrm{Ag}_{2} \mathrm{X}_{2}\left(\mathrm{PPh}_{3}\right)_{2}(\mu \text {-bpy) }]_{n}(\mathrm{a})\right.$; (b): $\left[\mathrm{Ag}_{2} \mathrm{I}_{2}\left(\mathrm{PPh}_{3}\right)_{2}(\mathrm{bpy})_{2}\right](1-\mathrm{I})$; (c): [py$\left\{\mathrm{Ag}_{2} \mathrm{X}_{2}\left(\mathrm{PPh}_{3}\right)_{2}\right\}$-bpy- $\left\{\mathrm{Ag}_{2} \mathrm{X}_{2}\left(\mathrm{PPh}_{3}\right)_{2}\right\}$-py] (2-X); (d) [\{( $\left.\left.\mathrm{NH}_{3}\right)\left(\mathrm{PH}_{3}\right)\left(\mathrm{Ag}_{2} \mathrm{X}_{2}\right)\left(\mathrm{PPh}_{3}\right)\right\}$-bpy$\left.\left\{\left(\mathrm{PPh}_{3}\right)\left(\mathrm{Ag}_{2} \mathrm{X}_{2}\right)\left(\mathrm{NH}_{3}\right)\left(\mathrm{PH}_{3}\right)\right\}\right](\mathbf{3}-\mathbf{X}, \mathbf{4 - C l})$. 


\section{REFERENCES}

S1) Shibata, S.; Tsuge, K.; Sasaki, Y.; Ishizaka, S.; Kitamura, N. Directional Energy Transfer in Mixed-Metallic Copper(I)-Silver(I) Coordination Polymers with Strong Luminescence. Inorg. Chem., 2015, 54, 9733-9739. 